\title{
Educational interventions involving physical manipulatives for improving children's learning and development: a scoping review
}

\author{
A PEDAL Report, January 2022
}

Authors \& contributors: Dr. Elizabeth M. Byrne ${ }^{1 *}$; Prof. Paul G. Ramchandani1; Kayleigh Skene'; Thomas Chupein²; Dr. Hanne Jensen²; Dr. Celia Hsiao²; Dr. Bo Stjerne Thomsen²; Dr. Amy Jo Dowd ${ }^{2}$

1 The Centre for Play in Education, Development, \& Learning (PEDAL),

Faculty of Education, University of Cambridge, UK

2 The LEGO Foundation, Billund, Denmark

*Corresponding author; emb72@cam.ac.uk

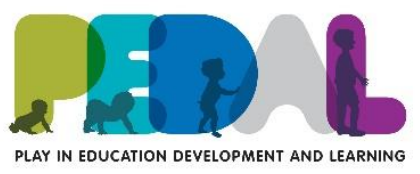




\section{Contents}

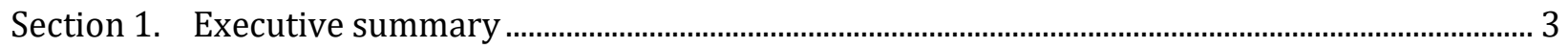

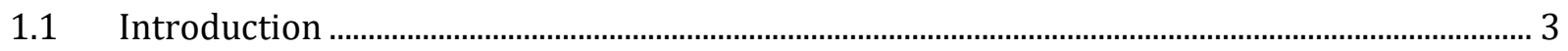

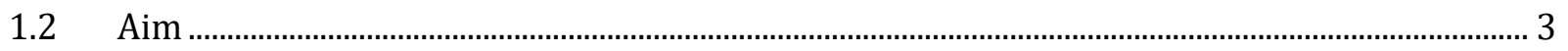

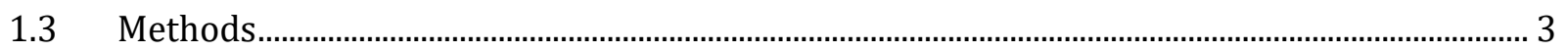

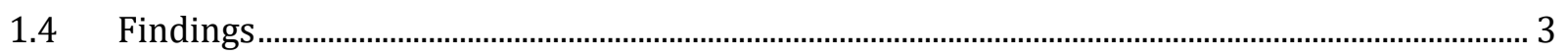

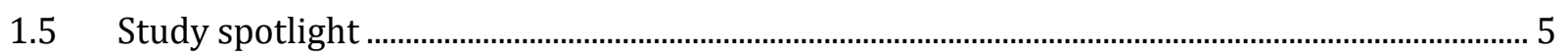

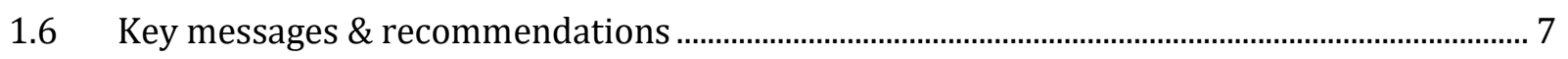

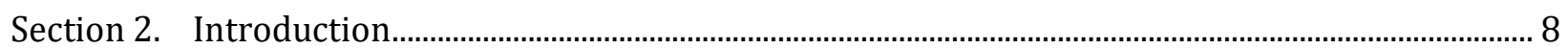

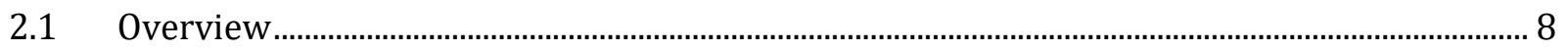

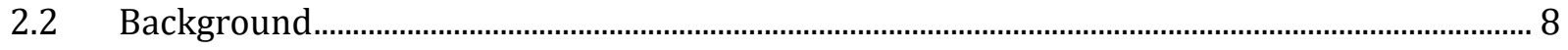

2.2.1 The developmental benefits of early object play..................................................................... 8

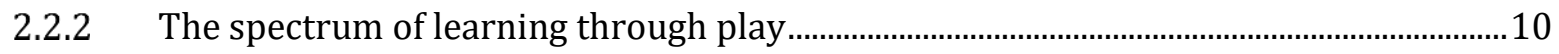

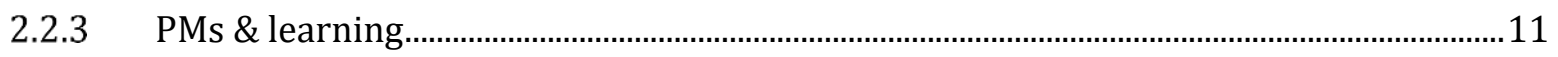

2.2.4 Existing reviews \& gaps in the literature............................................................................. 12

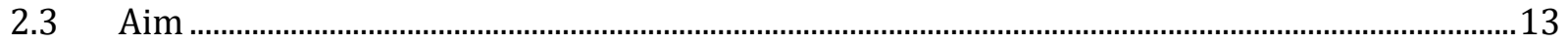

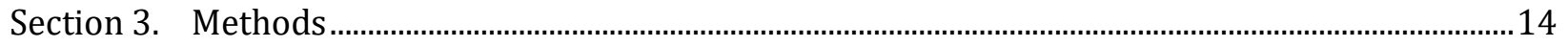

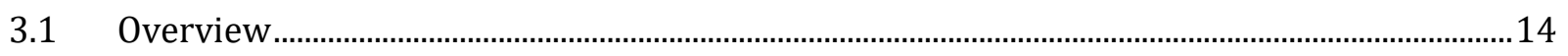

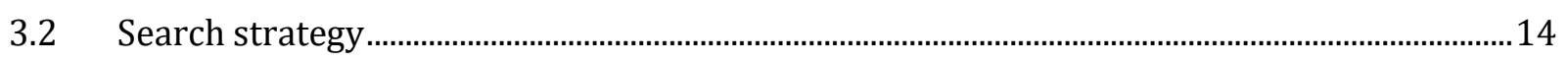

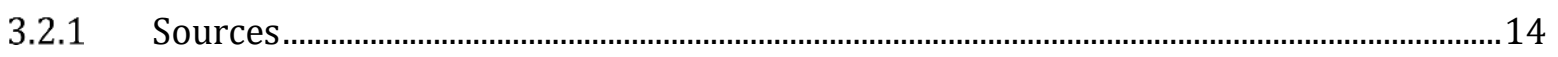

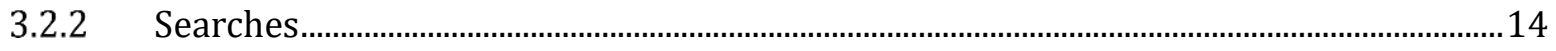

3.3 Study screening \& selection................................................................................................... 14

3.4 Charting \& summarising the data.....................................................................................................

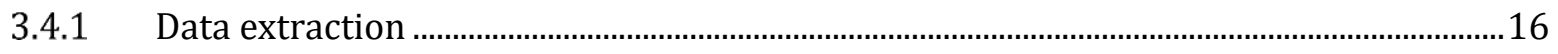

3.4.2 Data synthesis ......................................................................................................................... 17

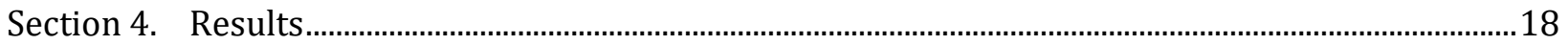

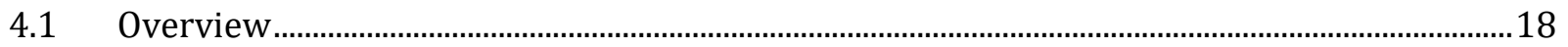

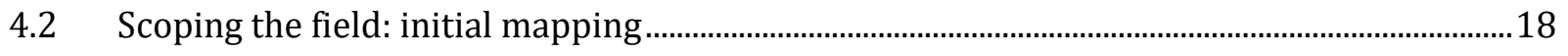

4.2.1 Study demographics \& characteristics .................................................................................18

4.2.2 Level of instructional guidance \& play................................................................................. 21

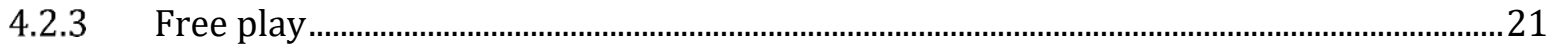

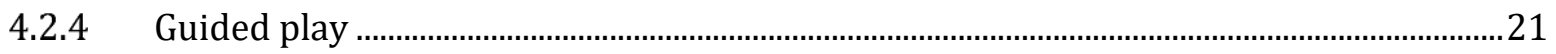




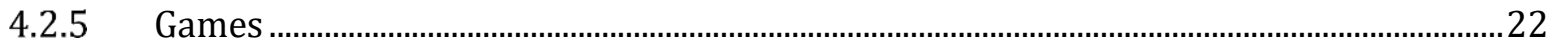

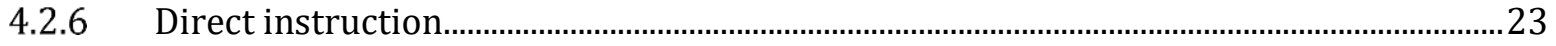

4.3 Review of interventions involving physical manipulatives (PMs) ..........................................24

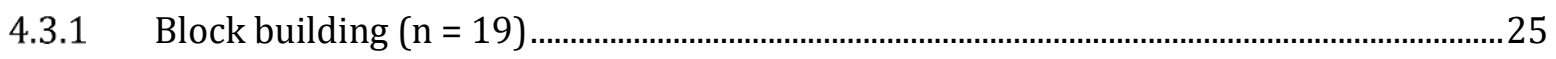

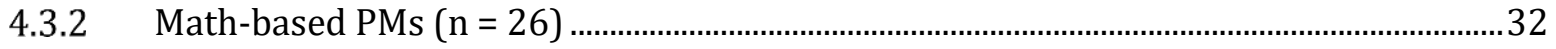

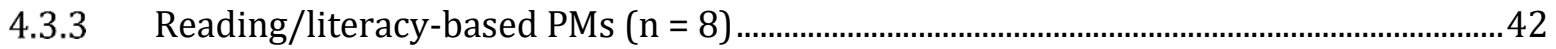

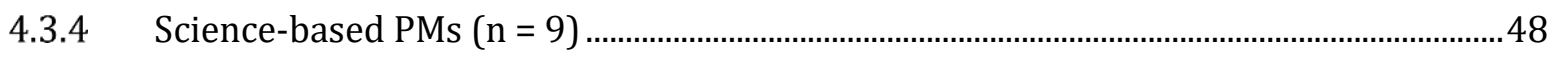

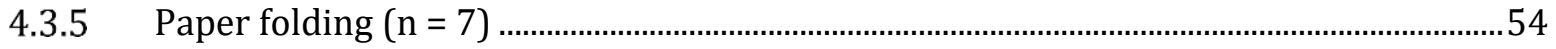

4.3.6 Puzzles \& Tangrams $(n=6)$

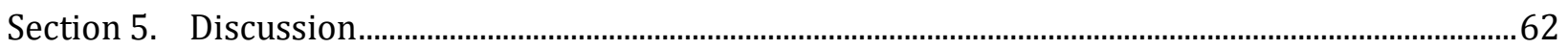

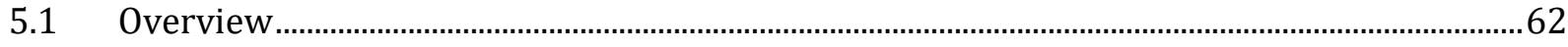

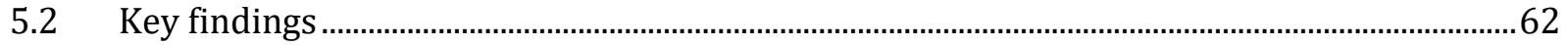

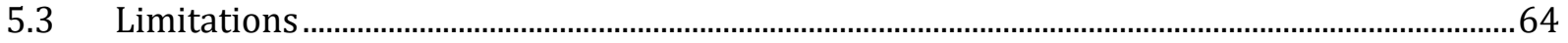

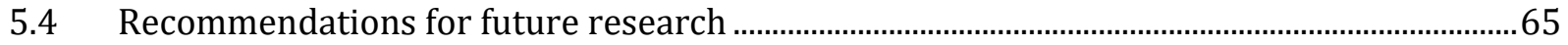

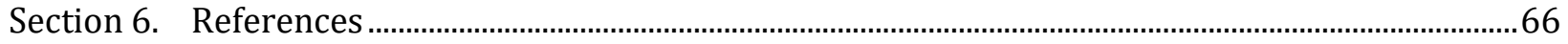

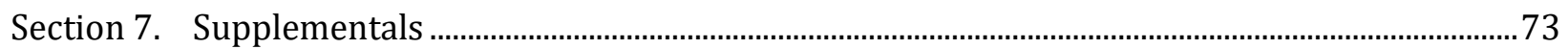

7.1 Appendix A. Search terms and syntax ……...................................................................................

7.2 Appendix B: Screening codes and guidance..................................................................................... 74

7.3 Appendix C: Data extraction codes ………….............................................................................

7.4 Appendix D: Main outcome domains and findings by study grouping ......................................78

7.4.1 Block building ................................................................................................................... 78

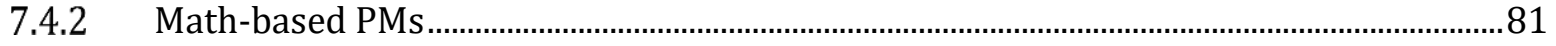

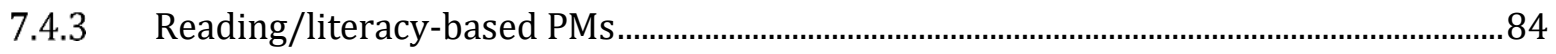

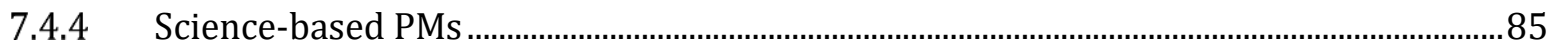

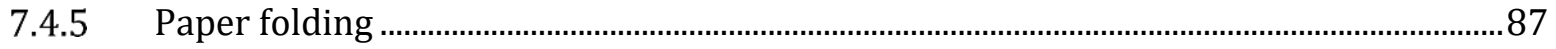

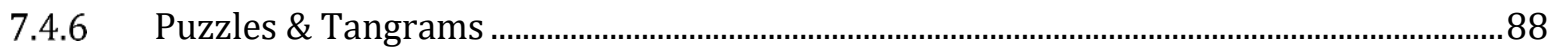

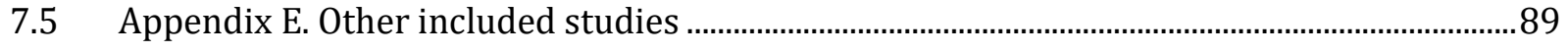

7.6 Appendix F: References of included studies ………………..........................................................92

7.7 Appendix G: Potentially relevant studies excluded due to insufficient information........... 100 


\section{Section 1. Executive summary}

\subsection{Introduction}

Physical manipulatives (PMs) are concrete objects that children use during hands-on learning activities. PMs can be useful educational tools as they promote children's active participation in learning and can facilitate playful hands-on experiences. PMs are widely used during math instruction, and there is a growing body of research for their use in other learning domains, often in a play-based context.

\subsection{Aim}

The current review sought to comprehensively map and synthesise the research literature on educational interventions involving PMs for young children, and to identify gaps in the research.

\subsection{Methods}

A scoping review methodology was employed. A search strategy was developed to search peerreviewed literature and unpublished reports (e.g., theses) indexed by electronic databases, covering the period 2000-2020. Following the removal of duplicates, the search yielded 3,112 records, all of which were screened based on their titles and abstracts. Next, full text reports of 918 studies were assessed for eligibility, which ultimately resulted in 102 studies being included in the review. For inclusion, studies must have satisfied various eligibility criteria, including (but not limited to), having a mean sample age between 0-12 years and at least one measurable child outcome. Data were extracted from all included studies and categories were developed to aid synthesis of the available evidence by the type of PMs used in the intervention and/or the learning domain targeted.

\subsection{Findings}

Studies were largely conducted in high- or upper-middle-income contexts ( $97 \%)$, primarily in the USA ( $\sim 59 \%)$. Most involved a sample of children with a mean age between 4-6 years $(\sim 52 \%)$ and took place in a school setting $(\sim 78 \%)$. The interventions varied greatly in terms of: (a) the PMs that children engaged with, (b) the hands-on activities PMs were used in, (c) the amount of physicality both the PMs and activities afforded children, (d) the degree of adult control and child autonomy (e.g., whether interventions were play-based or didactic), (e) the research methodologies used, and (f) the learning domains targeted (see Figure 1 for a visual summary of these differences).

Overall, findings relating to effectiveness were mixed. Some positive effects were reported for children's math and spatial outcomes following interventions involving block building, math-based PMs, puzzles, and paper folding. Benefits were also found to children's vocabulary and literacy skills following reading/literacy-based interventions with PMs. However, there were also numerous reports of null, negative, and inconclusive results, in part due to methodological inconsistencies and shortcomings across studies, meaning that some caution should be exercised when drawing firm conclusions. 


\section{INTERVENTIONS}

\section{What did children experience in the PM-based programmes?}

\section{MATERIALS}

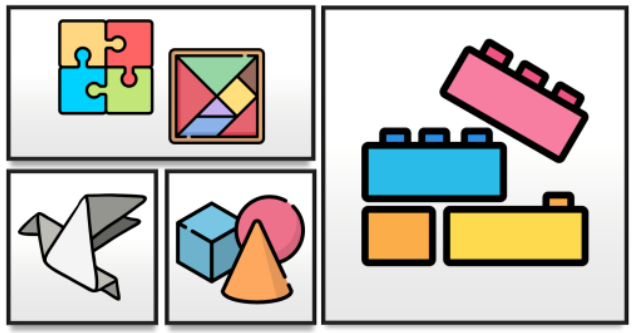

Children engaged with a wide range of physical manipulatives (PMs), such as blocks, bricks, puzzles, math materials like counters \& fraction tiles/pies, shapes, paper, \& small toys/figurines.

\section{LEVEL OF INSTRUCTION}

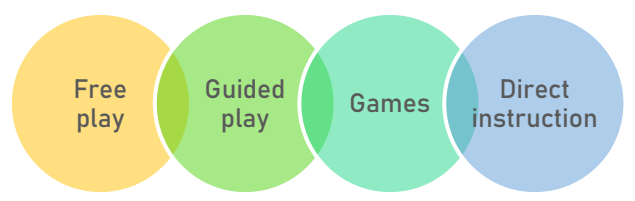

Some interventions were play-based - affording children freedom \& choice within the activities with gentle adult guidance \& scaffolding (i.e., guided play), whereas others were highly structured \& directed by an adult.

\section{ACTIVITIES}

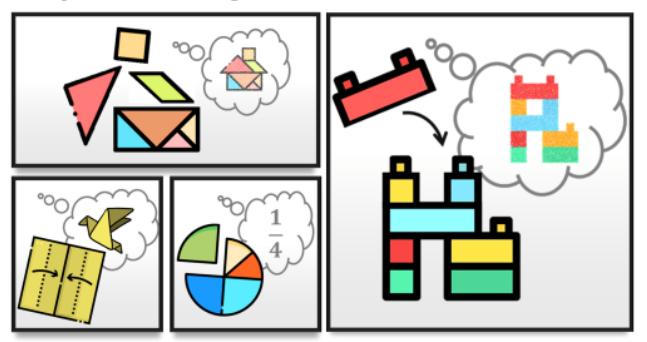

The ways that children used the materials also varied greatly. PMs were used in hands-on activities such as building, counting, origami, making patterns, enacting stories, scientific experimentation, \& solving math problems.

\section{LEVEL OF PHYSICAL ENGAGEMENT}

The level of physicality

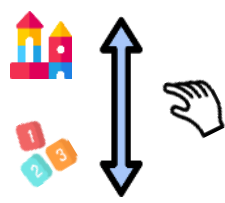

afforded by the materials \& activities differed. For example, children taking part in block play \& origami interventions engaged in high levels of physical manipulation, whereas activities such as shape sorting or counting with tokens involved lower levels.

\section{STUDIES}

\section{How did studies assess the effectiveness of interventions?}

\section{RESEARCH METHODOLOGIES \\ LEARNING DOMAINS}
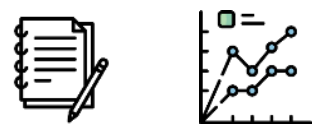

Methods used to evaluate interventions varied. Most studies included a comparison group (e.g., intervention compared to business-as-usual, guided play compared to direct instruction/free play, physical versus virtual materials). While some studies had robust research designs, many were limited by methodological issues.
The PM-based

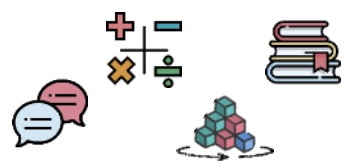
programmes targeted a range of learning domains. Consequently, many different outcome measures were used across the studies, which related to areas such as such as math \& numeracy (e.g., general math ability, fraction knowledge), literacy \& language (e.g., expressive $\&$ receptive vocabulary, reading), and visualspatial skills (e.g., mental rotation).

Figure 1. Infographic summarising of some of the broad differences across the reports included in this review, in terms of the interventions delivered to children, and in the way that studies examined these interventions ${ }^{1}$.

${ }_{1}^{1}$ The images used in the figure, some of which have been adapted/edited, are attributable to the following designers: Darius Dan; Freepik; juicy_fish; Muhammad Ali; photo3idea_studio; Pixel perfect; \& Smallikeart (from Flaticon). 


\subsection{Study spotlight}

Some examples of interventions with robust research designs that demonstrated particularly promising findings are highlighted in this section, areas for further exploration are also suggested.

AN INQUIRY-BASED MATH INTERVENTION IN BELIZE

Hull et al., (2018)

Math instruction in Belize typically involves highly prescribed activities (e.g., drill, memorisation), and hands-on learning with physical manipulatives (PMs) is not common practice. In this study, a multifaceted intervention was designed to directly meet the key needs of primary education in Belize, specifically addressing teacher knowledge and school resources. The different components of the intervention included inquiry-based instruction, hands-on activities with cost-effective (homemade) PMs, teacher professional development, and ongoing support for teachers. The programme was wellthought-out and grounded in educational theory. While the programme itself was novel, the authors consolidated existing evidence for the inclusion of each component. After a large-scale year-long trial, the programme was found to enhance children's overall math achievement compared to a control group. The positive results supported a nationwide rollout of the intervention.

An adaptation of this programme may benefit children's math learning in similar contexts, namely low- and middle-income countries where math is usually taught through direct instruction. The study provides evidence that children in a traditionally teacher-centred context respond well to a relatively simple, inexpensive programme that promotes child-centred learning via hands-on activities.

The programme demonstrates good practice for producing stronger and better data, in terms of intervention development (e.g., designed to meet the specific needs of a certain educational context, grounded in existing evidence), and trial design (e.g., robust methodology \& analyses). Both contribute to the success of an intervention and its potential impact on real-world policy and practice. 
A PLAYFUL PRE-SCHOOL MATH INTERVENTION IN THE USA

Sophian (2004)

In this study, young preschool children engaged in a playful, curriculum-based math intervention. It was delivered mostly by their teachers, but also involved some at-home activities with parents/caregivers. Like Hull et al., (2018), teachers received training and ongoing support throughout programme delivery.

The curriculum was varied and involved many different PMs, activities, and games. There were different themes each week that focused on different math concepts such as geometry/shapes, measurement, and numbers. Within each theme there were lots of related activities, for example, sorting small toys by size, filling different containers with beans/sand, or using plastic shape tiles to make/fill bigger shapes.

The study had a robust research design and results indicated that the programme benefited children's math ability scores more than control conditions. The results demonstrate how young children's math learning can be supported via teacher-mediated hands-on activities.

Future studies could serve to further test or extend the programme. For example, to examine whether the benefits are replicable in similar contexts, or in novel contexts following adaptation, and to explore whether the programme can be made more cost-effective for potential scaling in lower-resourced settings (e.g., using low-cost materials that teachers could make themselves).

\section{READ-PLAY-LEARN: AN ENRICHED BOOK-READING INTERVENTION IN THE USA}

\section{Toub et al., (2018)}

The study aimed to promote pre-school children's language learning through bookreading and play. Children engaged in eight shared book-reading sessions in small groups with an adult researcher. Afterwards, children played with story-relevant materials (e.g., small toys and figurines). Some children received adult support during play: guided play or directed play. In the former, the adult followed the children's lead and incorporated target words at naturally occurring moments. In the latter, the adult used a script with target words and directed children to re-enact the story. Another group of children used the toys however they liked without adult support of vocabulary (free play).

The study was well-designed and produced some promising results: children who received either type of adult support (guided or directed) improved more than those in the free play group on vocabulary measures involving the target words. Additional data from a second study also provided preliminary evidence in support of programme implementation by teachers.

Future work could further explore whether the intervention can be facilitated by teachers instead of researchers. Adaptations of the programme could be piloted to encourage play-based language learning. While PMs are widely used for learning math, they are less common during literacy-based instruction. Research evidence about PM-based literacy interventions is increasing gradually, and further development of programmes in this domain may prove fruitful. 


\subsection{Key messages \& recommendations}

\section{Key message 1 | Geographical diversity}

Studies were overwhelmingly conducted in high-income countries, with over half taking place in the USA.

\section{Recommendation}

Conduct more research in lower-income countries. Focus on implementing and scaling interventions that have an evidence base, or design interventions with components that have pre-existing evidence. Piloting must precede implementation and scaling if the evidence is mainly derived from high-income countries. Ensure programmes meet context-specific needs and that adaptations are made so they are useful, acceptable, and feasible. Design methodologically rigorous studies to ensure that effective interventions have strong and reliable data to support scaling and potential impact on policy.

\section{Key message 2 | Evidence relating to effectiveness \& methodological quality}

The literature spanned many different types of interventions and PMs, and the research evidence was mixed. Whilst there were positive findings for some interventions, others showed no effects. Several larger-scale studies with robust designs were identified, but many others were limited by methodological issues, such as small samples, lack of control groups, lack of baseline data, and/or inadequate statistical analyses. Methodological differences between studies mean that overall conclusions on the effectiveness of PMs should be drawn with some caution.

\section{Recommendation}

Recognise that there is a diverse literature for PMs. Conduct high quality and clearly focussed research that will allow stronger conclusions to be drawn in the future. Ensure that future intervention studies have robust research designs (e.g., that have control groups, pre- and post-intervention data - including transfer measures, prespecified protocols that outline key research questions and methods, and an analysis plan describing how the research questions will be addressed). In addition, a more targeted and systematic examination of the literature may be warranted, as the aggregation of data from the most methodologically robust and large-scale trials may yield firmer conclusions on the overall efficacy of PM-based interventions. 


\section{Section 2. Introduction}

\subsection{Overview}

Early childhood education (ECE) is a critical window of opportunity for supporting children's learning and development. Better quality ECE leads to better outcomes and prospects over the lifespan, and consequently, there have been calls for global action to invest in ECE (e.g., UNICEF, 2019). To ensure that young children have a strong foundation for lifelong learning, educators must provide them with enriching, engaging, and age-appropriate learning experiences and opportunities, and research must establish promising areas in which to invest.

There is a growing body of research examining the effectiveness of interventions involving physical manipulatives (PMs) for children's learning. PMs are concrete/tangible objects that children physically interact with during hands-on learning activities (e.g., building blocks, fraction tiles, counters), and are widely used during primary-school teaching. Didactic and play-based interventions involving PMs have been developed to facilitate children's learning (Carbonneau et al., 2013; Skene et al., 2021), however research related to their efficacy is atomised, with large variation across intervention studies in terms of the types of PMs used, the level of adult guidance/child choice, and the learning domains targeted. PM-based interventions have typically focused on children's math skills (for reviews, see Lafay et al., 2019; Sarama \& Clements, 2009), but there is emerging (yet more limited) research on the benefits of hands-on learning in other domains such as reading, spatial cognition, and science (e.g., Toub et al., 2018; van Schijndel et al., 2010; Vander Heyden et al., 2017).

This scoping review aimed to provide a comprehensive overview of the current research on educational interventions involving PMs - regardless of learning domain targeted or type of instruction used - and identify gaps in the research.

\subsection{Background}

\subsubsection{The developmental benefits of early object play}

There is evidence that play, including object play, benefits children's learning and development (for overviews, see Pellegrini \& Gustafson, 2005; Whitebread, 2012, 2019). Infants are naturally curious, and first start to explore the world using their senses. As soon as they can grasp and hold items, they engage in early exploratory and manipulative behaviours such as mouthing, hitting, dropping, and stroking (i.e., sensorimotor play) - investigating how objects feel and behave. By age two, children start arranging and then sorting objects, and by age four building and construction behaviours emerge (Whitebread, 2012). Physical play with objects benefits children's physical development by fostering fundamental skills such as fine and gross motor control and hand-eye coordination. But early manipulative play may have more far-reaching benefits for young children, beyond physical development, including spatial skills and later academic performance in school.

Spatial reasoning is an important cognitive ability that is used to mentally transform (e.g., rotate) information about objects in the mind and encode information about objects. It is fundamental to learning and important for many everyday activities, such as remembering locations of objects and 
events. Evidence shows that manual exploration of objects benefits infants' mental rotation ability (Möhring \& Frick, 2013), and correlational studies have established strong links between early play with PMs (like blocks and puzzles) and children's spatial reasoning (Caldera et al., 1999; Jirout \& Newcombe, 2015; Levine et al., 2012). In activities like block play, children imagine how blocks can fit together and be arranged; as well as manipulating objects physically, children also manipulate them mentally, thus tapping into and practicing visual-spatial skills. As young children handle and play with various toys and objects, they also start learning about concepts such as shape, size, weight, and space - laying the foundations of spatial and mathematical thinking (Botha, Maree, \& de Witt, 2005; Charlesworth \& Lind, 2003; Pound, 2006).

Early spatial experiences are also thought to impact children's school readiness (Verdine, Golinkoff, Hirsh-Pasek, \& Newcombe, 2014). Early play with PMs like building blocks positively predicts later math achievement in school (Wolfgang et al., 2001, 2003), and young children's block building complexity is associated with their concurrent spatial and math skills (Bower et al., 2020). There is also a well-established relationship between young children's spatial cognition and math ability (Casey et al., 2012; Clements \& Sarama, 2008; Gunderson et al., 2012; Mix et al., 2016; Mix \& Cheng, 2012; Verdine, Golinkoff, Hirsh-Pasek, Newcombe, et al., 2014). Spatial reasoning is one of the strongest predictors of young children's math achievement in primary school (Fernández-Méndez et al., 2020; Mix \& Cheng, 2012), and may support math learning of concepts such as geometry, mental arithmetic, magnitude estimation, counting, and algebra (Battista, 1990; Kyttälä et al., 2003; Kyttälä \& Lehto, 2008; Thompson et al., 2013; Tolar et al., 2009). Furthermore, spatial ability also positively predicts whether children will enjoy and succeed in STEM-based subjects, and whether a person will pursue a STEM-related career in the future (Khine, 2016; Wai et al., 2009).

Promising evidence suggests that spatial skills are malleable, for example, meta-analytic data has shown that spatial training can improve spatial ability over the lifespan (Uttal et al., 2013). Based on these findings and the strong associations between spatial cognition and math, PM-based interventions targeting children's spatial skills, may in turn positively impact on their math skills. Furthermore, math competency in preschool children is a strong predictor of later school achievement (compared to reading and attention skills; Duncan et al. 2007) and labour-market success in adulthood (Ritchie \& Bates, 2013). Therefore, interventions that improve children's math skills, could in consequence have potentially long-lasting benefits over the lifespan.

Children's early object play may also play a unique role in fostering the development of children's problem solving skills (Cheyne \& Rubin, 1983). Pellegrini and Gustafson (2005) gathered observational data showing that the amount of object and construction play that 3-5-year-olds engaged during a year at nursery, positively predicted their performance in problem-solving tasks (specifically, tasks that required them to use objects as tools), even after controlling for spatial skills. These findings suggest that construction play provides young children with opportunities to practice and develop their thinking skills. For example, when building a tower, children develop flexibility of thought as they iteratively try out different ideas through trial-and-error to achieve their goal.

Engaging in PM-based play activities like block play with peers, also provides children with opportunities for developing their language and social-emotional skills as they communicate and collaborate with each other - learning new vocabulary and practicing turn-taking and sharing. Object-based play also encourages children's creativity and imagination, for example, blocks can be 
combined in countless ways to create novel structures, and during sociodramatic play, children may imagine objects as representing something else in their mind. This review aimed to map the available evidence for key child outcomes assessed in PM-based intervention studies.

\subsubsection{The spectrum of learning through play}

Over the last decade there has been a growing body of research on, and debate surrounding, playbased pedagogy (Fisher et al., 2012; Hirsh-Pasek et al., 2010; Pyle et al., 2017; Pyle \& Danniels, 2017; Skene et al., 2021; Weisberg et al., 2016; Yu et al., 2018; Zosh et al., 2017, 2018). There has been a conceptual shift from didactic to play-based educational approaches, resulting in play being redefined as a continuum (see Figure 2; Zosh et al., 2018). Along this spectrum the different types of instruction afford varying degrees of child choice and adult involvement. At one end is free play-play that is child-initiated and -led, without an extrinsic goal, and at the other end is direct instruction - a non-playful approach that is adult-directed with an explicit learning goal. Between the two ends of the spectrum are guided play and games.

Guided play encourages children's active participation and involvement in learning through play (Zosh et al., 2018). Core features of guided play include: (a) child choice (children have some degree of freedom and choice over their own actions and play), (b) adult guidance (adults initiate playful activities and scaffold children using techniques such as prompts, co-play, open-ended questions, modelling behaviours/actions, setting challenges, and adjusting to the specific needs and interests of children), and (c) a learning goal (play-based tasks have a clear learning goal which the adult gently steers children towards; Skene et al., 2021; Weisberg et al., 2013b).

Games are also adult-initiated and child-directed but afford children relatively less freedom within the learning activity: (educational) games are typically adult-designed, have an explicit goal, and are rule-based. However, by defining play along a continuum, it can be inherently difficult to distinguish between the distinct pedagogical approaches. For example, the level of child-choice and adultinvolvement also varies between guided play interventions, meaning that it can be difficult to determine where guided play ends and free play or direct instruction begins.

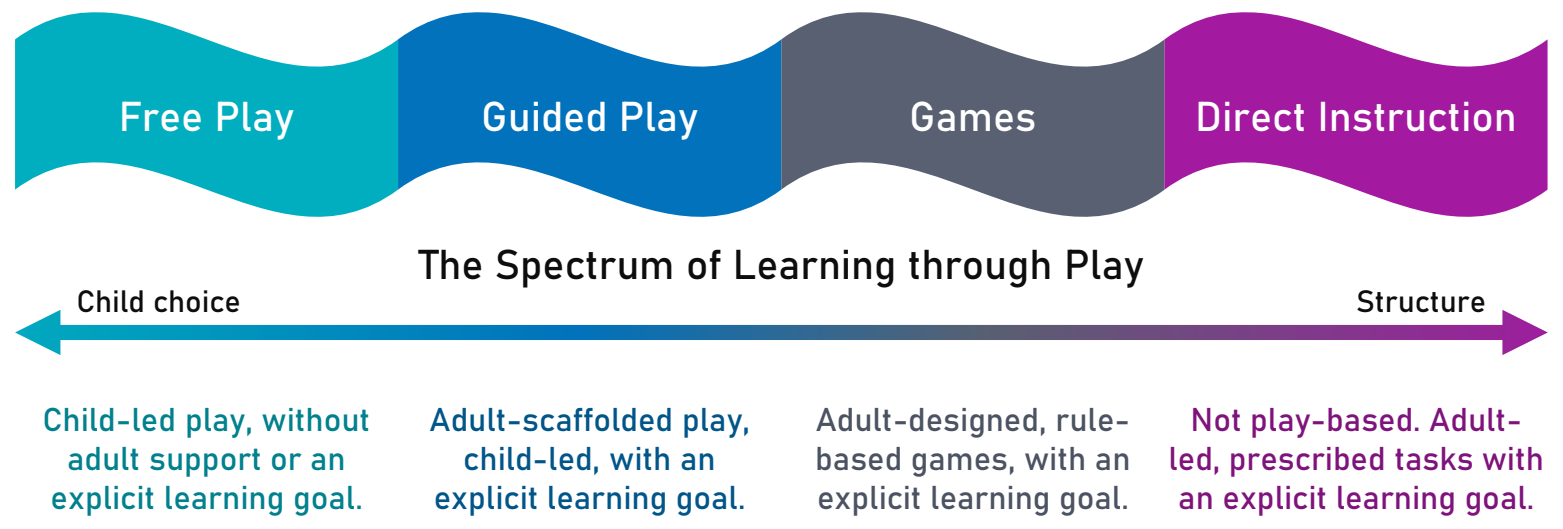

Figure 2. The spectrum of learning through play (adapted from Zosh et al., 2018). Free play, guided play, and games fall under the umbrella of play-based learning, whereas direct instruction is not play-based. Along the continuum, there are varying degrees of child-adult involvement. 
There is no consensus on the optimal level of instructional guidance. In the wider field, an adultscaffolded and child-/learner-centred approach is supported by meta-analytic data, which indicates that unassisted-discovery results in poor learning. Alfieri et al. (2010) found that explicit instruction (traditional teaching) was more effective than unassisted discovery-based learning (learning through independent exploration), and that enhanced/assisted discovery-based learning was better than other types of instruction (e.g., explicit, unassisted discovery). The findings suggested that pupils benefited from feedback, scaffolding, worked examples, and elicited explanations, and that some level of instructional support or guidance facilitates learning (Alfieri et al., 2011). Similarly, a review found that guided-discovery was better than unassisted-discovery for learning and transfer (Mayer, 2004). The cognitive load theory may explain these findings, which suggests that guidance and support provided by a teacher lowers the cognitive load placed on a child's limited working memory capacity (and other executive functions; Alfieri et al., 2010; Kirschner, Sweller, \& Clark, 2010). Evidence supporting the combination of PMs with a guided-play approach for math instruction is provided by Cohen (1984). Children who were encouraged to follow their own interests when manipulating objects, learned more than when each of their movements was directed by a teacher, suggesting that while teachers should take an active role when supporting pupils' use of PMs in lessons, they should act as guides - providing support and encouraging children to use and view PMs in different ways (Cohen, 1984).

Literature on guided play is emerging, with mixed evidence for its benefits. A recent systematic review and meta-analysis found that guided play interventions benefited children's early math skills, shape knowledge, and task switching skills more than direct instruction, and had a greater positive effect than free play on spatial talk (Skene et al., 2021). However, no differences were found for other numeracy, literacy, executive function, or social-emotional outcomes. The review also revealed a large degree of heterogeneity in how guided play was conceptualised and implemented across studies (i.e., how much authors acknowledged key elements that characterise guided play, and to what extent the intervention activities that were delivered were aligned with the core principles of guided play, respectively).

\subsubsection{PMs \& learning}

Evidence suggests that PMs are effective educational tools for classroom learning. Most research has focussed on math instruction, during which PMs such as such as cubes, blocks, tiles, rods, and counters are commonly used (Carbonneau et al., 2013; Furner \& Worrell, 2017; Mix et al., 2017; Moyer \& Jones, 2004). PMs may also be effective tools for teaching in other domains, including reading and science, however these areas are less researched.

It is widely accepted that PMs benefit children's math achievement, with multiple reviews and metaanalyses demonstrating positive effects following math interventions involving PMs (Carbonneau et al., 2013; Holmes, 2013; Lafay et al., 2019). PMs are thought to be particularly beneficial for math learning as they help children to strengthen their concrete thinking before moving on to more complex abstract (symbolic) ideas (Taylor \& Boyer, 2020), and helps them to relate learned math concepts to practical, real-word experiences (Holmes, 2013; Rittle-Johnson \& Koedinger, 2005). While there is strong evidence for the effectiveness of PMs for math instruction generally, the optimal 
level of instructional guidance has not been established ${ }^{2}$. Meta-analytic data demonstrated that higher levels of instructional guidance during PM-based math instruction were associated with greater student learning (Carbonneau et al., 2013). Though interestingly, lower levels of guidance were associated with larger effects of transfer of learning, suggesting that the children who learn effectively with limited adult support consequently develop a greater conceptual understanding, which in turn enables them to flexibly transfer their knowledge to novel circumstances (Carbonneau et al., 2013).

In another learning domain - reading/literacy - theories of embodied cognition suggest that physical manipulation facilitates children's reading comprehension (Glenberg, 2008). When a child manipulates objects corresponding to some text (e.g., physically interacting with a toy tractor or horse when reading a story about a farm) it forces indexing and derivation of meaning (i.e., it facilitates their acquisition of semantic information relating to words in the story; Glenberg et al., 2004). There is also support for PMs in science-based learning. There are many overlapping characteristics between play and scientific thinking, including curiosity, creativity, exploration, experimentation, invention, innovation, and discovery. Therefore, play-based experiences with PMs may encourage these traits and promote learning of science concepts. In support of this, Jarrett (1998) found that exposing trainee teachers to hands-on experiences influenced their enjoyment of science. Following their training, they also rated the PM activities as being fun, interesting, and high in learning potential. Jarrett (1998) stresses the importance of considering teachers' attitudes when developing classroom activities: if teachers perceive subjects like science as boring, difficult, and/or uninteresting, they are less likely to involve students in the fun of scientific inquiry. By promoting teachers' positive attitudes towards science through hands-on experiences, they may be more likely to implement them in their own teaching practice, and consequently convey a positive attitude towards science to their students.

\subsubsection{Existing reviews \& gaps in the literature}

Existing literature reviews that relate to the current project have primarily focused on examining the efficacy and effectiveness of PM-based interventions or have compared interventions with different levels of instructional guidance.

Previous literature reviews and meta-analyses of studies involving PMs have primarily focused on math instruction, and have examined the impact of math interventions with versus without ${ }^{3}$ PMs (e.g., Carbonneau et al., 2013; Holmes, 2013; Lafay et al., 2019), or compared the effects of physical (concrete) compared to virtual (digital) materials for math learning (Moyer-Packenham \& Westenskow, 2013; Sarama \& Clements, 2009; Tran et al., 2017). In a different learning domain, another review investigated the impact of PMs and illustrations during book reading on children's

\footnotetext{
${ }^{2}$ Furthermore, it is unclear whether play-based activities involving PMs will be effective and accepted by classroom teachers. Qualitative findings suggest that some teachers hold negative attitudes about PMs: perceiving them as frivolous as they encourage play and distract children from 'real' learning (Moyer \& Jones, 2004). For example, Moyer (2001) found that teachers who used PMs in math lessons reported them being fun, but not necessary for teaching and learning math. Notwithstanding, both teachers and students conveyed that math lessons involving PMs were more enjoyable (Moch, 2001; Moyer, 2001), resulting in higher (perceived) levels of pupil engagement and motivation (Moyer, 2001).

${ }^{3}$ E.g., Instruction that only used abstract math symbols or business-as-usual.
} 
literacy skills (Trivette et al., 2012). However, the report made no distinction between PMs and illustrations, and only a limited number of studies in the review involved PMs.

Several reviews have considered the effects of different types of instruction and guidance for children's learning. Alfieri et al. (2010) compared structured versus unstructured pedagogical approaches (see also, Kirschner et al., 2010), Pyle et al. (2017) conducted a scoping review of playbased teaching approaches, and Skene et al. (2021) recently undertook a systematic review of guided-play approaches in educational contexts. While some, but not all, studies included these reviews involved PMs, the presence of hands-on activities with concrete materials was not examined independently. While interventions included in these reviews may have incidentally involved PMs, they were not a focus of study in any, nor were variables related to physicality or concrete materials examined as moderators.

\subsection{Aim}

The current scoping review aimed to map the literature evaluating educational interventions and programmes involving PMs and differed from existent research in several ways. First, a broad range of literature studying the effects of educational interventions involving PMs was considered, regardless of the learning domain of focus/targeted (i.e., not limited to math-based programmes), or the pedagogical approach employed (e.g., not limited to didactic or play-based instruction). The review sought to further characterise the research and explore similarities and differences across an array of studies. Data related to study characteristics are summarised descriptively and quantitively in tables, and other factors of interest are discussed qualitatively using a narrative synthesis approach (e.g., the level of adult guidance and child autonomy, the degree to which children physically engaged with PMs). Finally, while some previous reviews do not distinguish between physical and virtual manipulatives (e.g., Bouck \& Park, 2018; Lafay et al., 2019), this review specifically focused on interventions involving physical (tangible, concrete) materials ${ }^{4}$.

${ }^{4}$ Including studies comparing physical and virtual manipulatives. 


\section{Section 3. Methods}

\subsection{Overview}

The methodological approach was informed by Arksey and O'Malley's (2005) framework for conducting scoping reviews (see also, Levac et al., 2010). A protocol was registered with the Open Science Framework (OSF; https://osf.io/p4jk9/). There were some deviations from the original plan, primarily due to the iterative process of conducting a scoping review and because of resource constraints. In the following sub-sections, the procedures used to identify, screen, and summarise the literature are described.

\subsection{Search strategy}

\subsubsection{Sources}

Searches were conducted of six bibliographic databases. They were selected following consultation with an information specialist and index a range of relevant psychology and education journals, as well as unpublished dissertations and theses. The following electronic databases were used: PsycINFO (EBSCO), Child Development and Adolescent Studies (EBSCO), ERIC (EBSCO), British Education Index (EBSCO), Scopus, and ProQuest Dissertations \& Theses.

\subsubsection{Searches}

Search terms corresponded to elements of the PCC (population, concept, context) framework (Peters et al., 2017), and were grouped into three categories: (1) population/context (primary and preprimary school age children), concept A (PMs), and concept B (intervention/programme). Search terms within each category were separated by the 'OR' Boolean operator, and categories were separated with the 'AND' operator. See section Appendix A (section 7.1) for further details regarding the search terms and syntax used. The title, abstract, and keyword fields were searched. The search was limited to papers published (or otherwise made available) between 2000-2020; no other limiters were used. Searches took place on 5 May 2020. Additional studies published after this date were also included if identified via hand search or provided by an author.

\subsection{Study screening \& selection}

The study selection procedure was conducted in accordance with the PRISMA guidelines for systematic reviews (Moher et al., 2009, 2015), that have been adapted for use with scoping reviews (Peters et al., 2015; Tricco et al., 2018). See Figure 3 for a flow diagram outlining the procedure for study screening and selection. Citations for all the reports identified in the search were imported into the EPPI-Reviewer web-based reference management software programme (version 4.0; Thomas et al., 2010), and duplicates were removed. Codes corresponding to the inclusion and exclusion criteria were created in the programme and used during the study screening phase. 


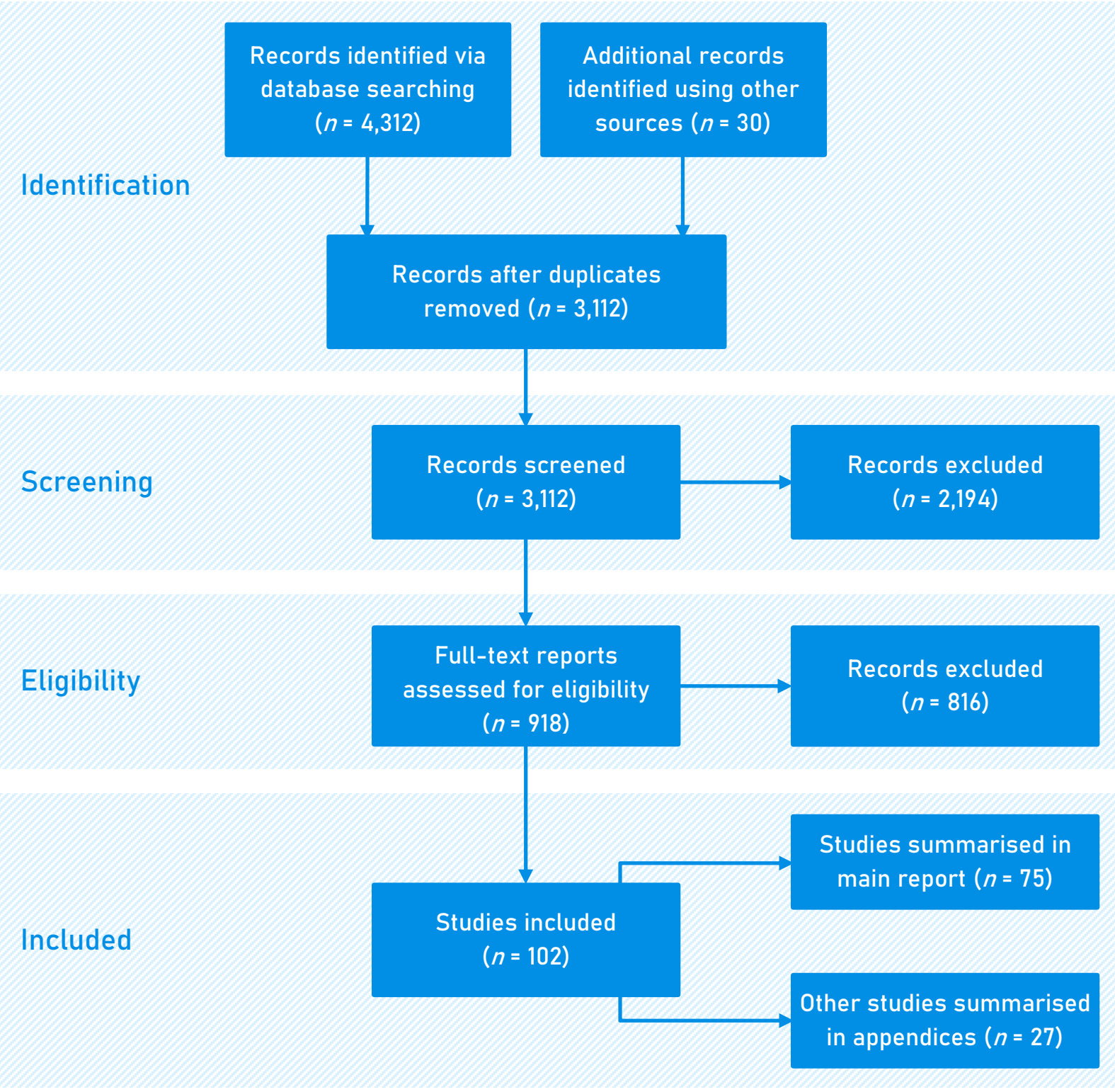

Figure 3. PRISMA search process flow diagram (adapted from Peters et al., 2015).

First, the title and abstracts off all identified reports were screened for inclusion using the following criteria (1-6):

1. Publication date: $2000-2020^{5}$

2. Language: English

3. Sample age: $0-12$ years

4. Study type: Empirical studies evaluating the impact of an intervention

5. Intervention: Educational interventions involving hands-on learning activities with PMs

${ }^{5}$ One report was published in 2021 [Tian]. Initially, a thesis was identified in the search and the first author subsequently provided a fulltext journal article of the same study following publication. 
6. Outcome: At least one clear and measurable child measure (related to learning or development)

Studies were excluded if they violated any of the above inclusion criterion. Studies were included for the second stage of screening if they met all the above criteria, or if more information was needed to determine their eligibility. Full text screening was then undertaken of this subgroup of studies using the codes above (1-6) plus the following criteria (7-11):

7. Full text: Available

8. Sample size: $\geq 20$

9. Focus of study: At least one intervention activity in which children physically engaged with PMs and that had a clear learning goal

10. Data: Quantitative

11. Materials: Concrete objects 6

For further details regarding the inclusion and exclusion criteria used to determine study eligibility, see Appendix A in the supplementals (Section 7.1). No restrictions were placed on the type of report (e.g., published journal article, PhD thesis, conference report, etc). The first author (EB) conducted screening of all reports at both stages. Prior to each stage, two authors (EB and KS) double screened a subset of $\sim 5 \%$ of reports $(n=156$ and $n=46$ at the title/abstract and full-text screening stages, respectively) ${ }^{7}$.

\subsection{Charting \& summarising the data}

\subsubsection{Data extraction}

The first author extracted data from the reports deemed eligible for inclusion. A descriptive-analytic method was used to guide extraction of information according to a data charting framework developed by the authors. Information was gathered on study location, participant characteristics, research design, intervention delivery, intervention materials and content, comparison group(s), main outcome domains, and main findings. See Table C1 in Appendix C (section 7.3) for an overview of the data charting form used. Data extraction codes were created in EPPI-Reviewer and data extracted were recorded in the programme.

Data were extracted from all included reports, where possible, by the first author (EB). Prior to this, two authors (EB and KS) conducted double screening on a $\sim 10 \%$ subset of reports $(n=10)^{8}$. Where information that was missing in the reports, attempts were made to contact the author(s) via email. A list of studies that did not provide sufficient information to determine eligibility, and were therefore excluded, is provided in Appendix G (section 7.7).

\footnotetext{
${ }^{6}$ Excluding writing and art-based materials used for writing or art-based activities (e.g., drawing, painting, etc.), purely virtual/digital materials, tangible-user-interfaces (TUIs, i.e., concrete objects used to engage with digital/virtual interfaces), and electronic materials.

7 The purpose of the double screening was to ensure that codes corresponding to the eligibility criteria were clear and appropriate. Discrepancies were discussed and the codes were adjusted accordingly.

${ }^{8}$ The purpose of the double screening was to ensure that codes corresponding to the to-be-extracted data were clear and appropriate. Discrepancies and difficulties that arose during the process were discussed, and the codes were adjusted accordingly (codes were also adjusted iteratively throughout the data extraction process).
} 


\subsubsection{Data synthesis}

First, included reports were broadly categorised according to the types of PMs used and/or learning domain targeted to aid synthesis of a large group of studies. Within each grouping of studies, a table of study characteristics was created, and a narrative synthesis approach was used to provide a descriptive overview of the interventions and reported findings. Using this approach, similarities and differences across studies were identified and discussed, along with methodological limitations and gaps in the research. Not all study groupings were included in the narrative synthesis - those deemed less relevant to the focus of the review are briefly summarised in the appendices. 


\section{Section 4. Results}

\subsection{Overview}

In total, 102 interventions involving PMs were included in the review. Most were journal publications $(\sim 78 \%)$, with the remainder being unpublished theses or dissertations $(\sim 19 \%)$, or other reports such as conference proceedings $(\sim 3 \%)$. A broad overview of all the studies, including study demographics and characteristics, and a summary of the different types of instructional guidance used, are provided in the following Section (4.2), Then, in Section 4.3, the interventions are grouped and discussed in more detail. In-text references to included reports are provided inside square brackets (e.g., [Hull]). Only the first author is provided, unless there are multiple reports of the same first author, in which case the publication year is also provided (e.g., [Fisher 2013]). A complete reference list corresponding to the included studies is provided in Appendix F (section 7.6).

\subsection{Scoping the field: initial mapping}

\subsubsection{Study demographics \& characteristics}

Figure 4 shows the number of research reports according to their sample age group. Children with a mean age between 4-6 years accounted for over half of the studies' samples ( $~ 53 \%)$.

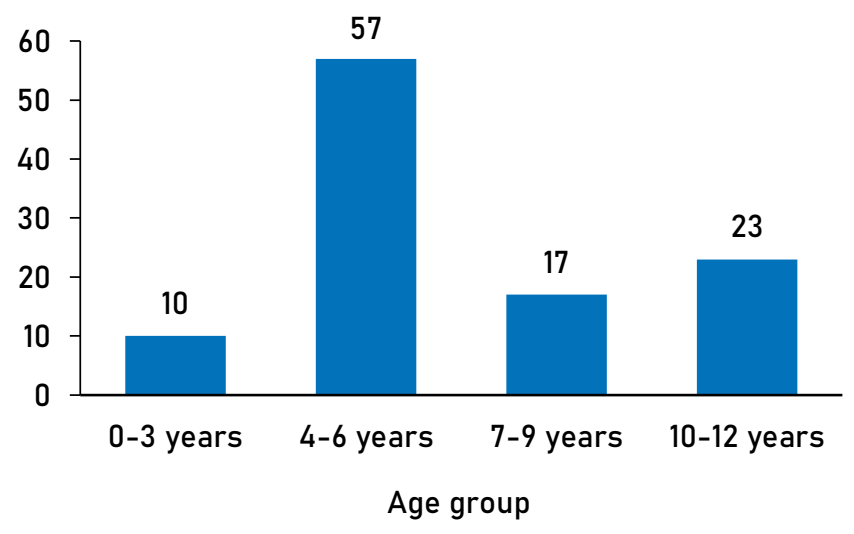

Figure 4. Number of studies by sample age group. Note. Several studies had groups of children in multiple age groups.

Figure 5 shows the geographical distribution of studies by the country they were conducted in. The interventions overwhelmingly took place in the USA ( 59\%). Overall, North America accounted for $\sim 62 \%$ of the studies, with Europe and the rest of the world accounting for the remainder $(\sim 17 \%$ and $\sim 21 \%$, respectively). 


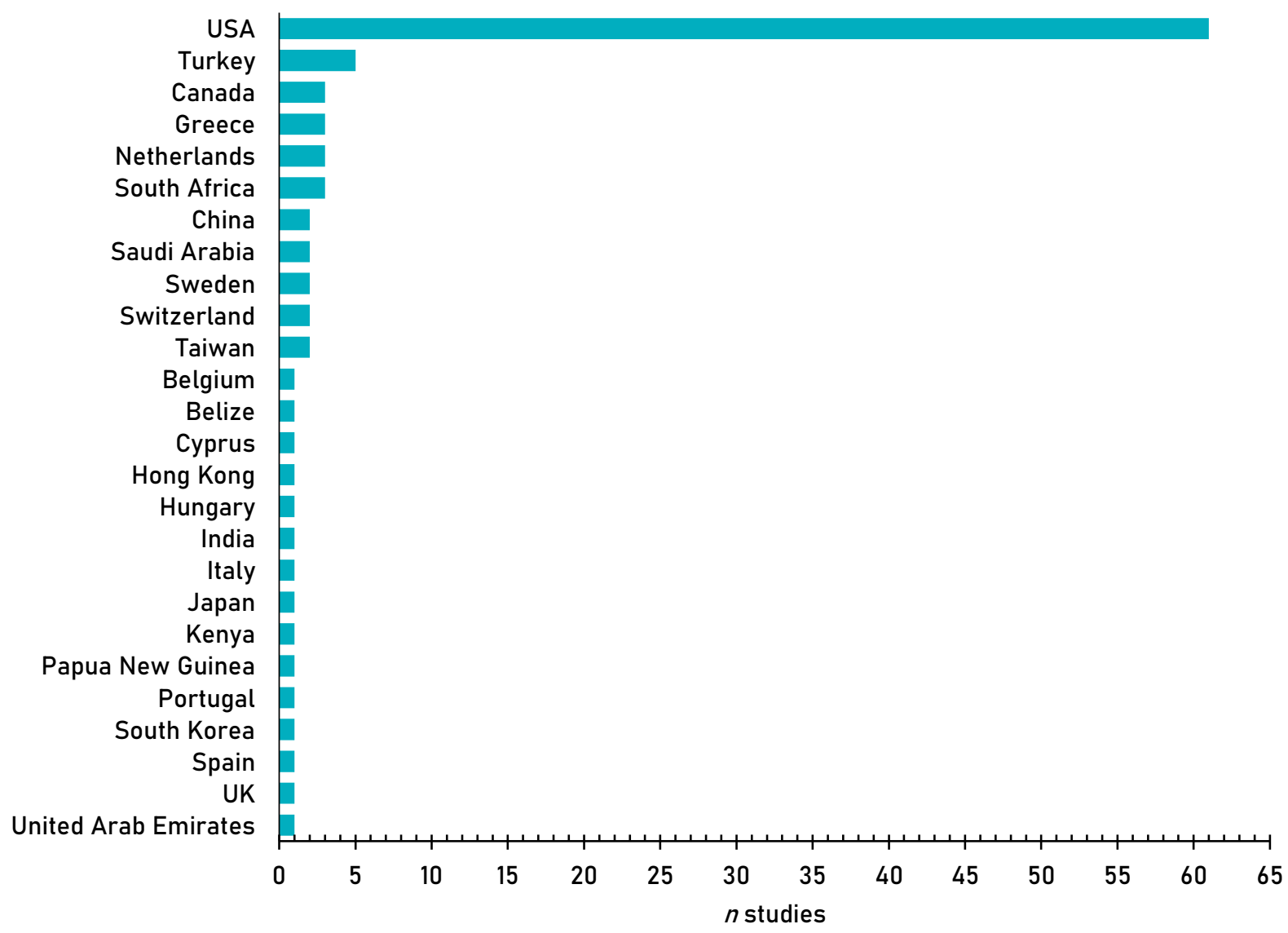

Figure 5. Geographical distribution of studies by country. Note. One study was conducted in two countries.

Figure 6 shows the proportion (\%) of studies by the gross national income (GNI; provided by The World Bank, 2021) of the country they were conducted in. Studies were largely conducted in highand upper-middle-income contexts ( $\sim 86 \%$ and $\sim 11 \%$, respectively) - this was primarily due to most studies taking place in the USA. Notably, only $\sim 3 \%$ were conducted in lower-middle-income countries and none took place in low-income contexts.

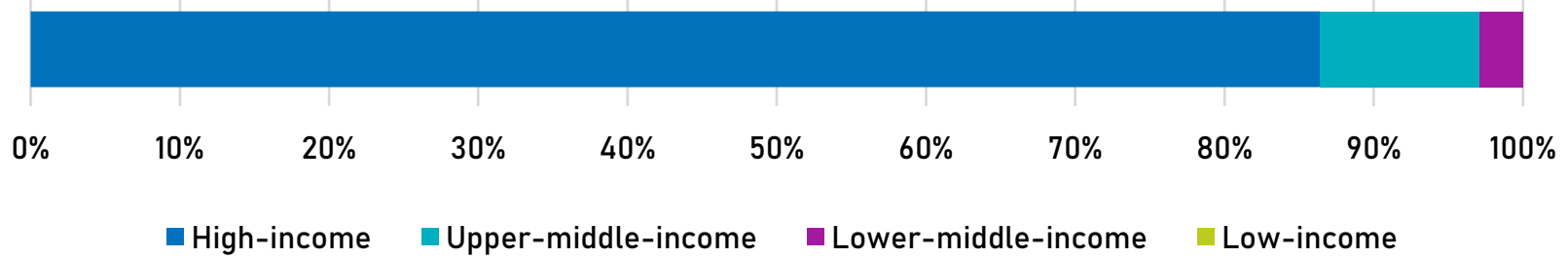

Figure 6. Proportional distribution of included studies by gross national income (GNI).

Figure 7 displays the distribution of studies according to several characteristics (including the setting, adult involved, research design, and test time points). As shown in panel A most interventions were conducted in a school setting $(\sim 78 \%)$. Despite this, the adult delivering the interventions was typically a researcher/experimenter $(\sim 46 \%)$, rather than a teacher at the school $(\sim 37 \%$; see Figure 
7, panel B). This may reflect differences in the purpose of the studies. While some implemented educational programmes and provided teacher training on how to deliver the interventions (e.g., Hull; Sophian), others - despite being conducted in an educational setting (i.e., a school) - were more aligned with lab-based experimental studies (e.g., children were taken from their classroom to a separate room for an individual or small-group session with an experimenter). For the latter cases, the school setting was somewhat incidental and enabled researchers to gather data from primaryschool-age children [e.g., Fisher 2013; Martin].

Most studies utilised a randomised controlled trial (RCT) design ( $\sim 60 \%$; see panel C, Figure 7$)$ debatably the 'gold standard' methodology (Hariton \& Locascio, 2018), or at least a good experimental design (Grossman \& Mackenzie, 2005; Lilienfeld et al., 2018), for evaluating if intervention programmes are effective. However, narrative synthesis of the included studies revealed substantial variation in methodological rigour across interventions, and various methodological inconsistencies and shortcomings are highlighted in the report. Finally, three studies did not include a comparison condition, and while some studies did not gather baseline data, most assessed children at both pre- and post-test time points ( $\sim 83 \%$; see panel C, Figure 7 ).
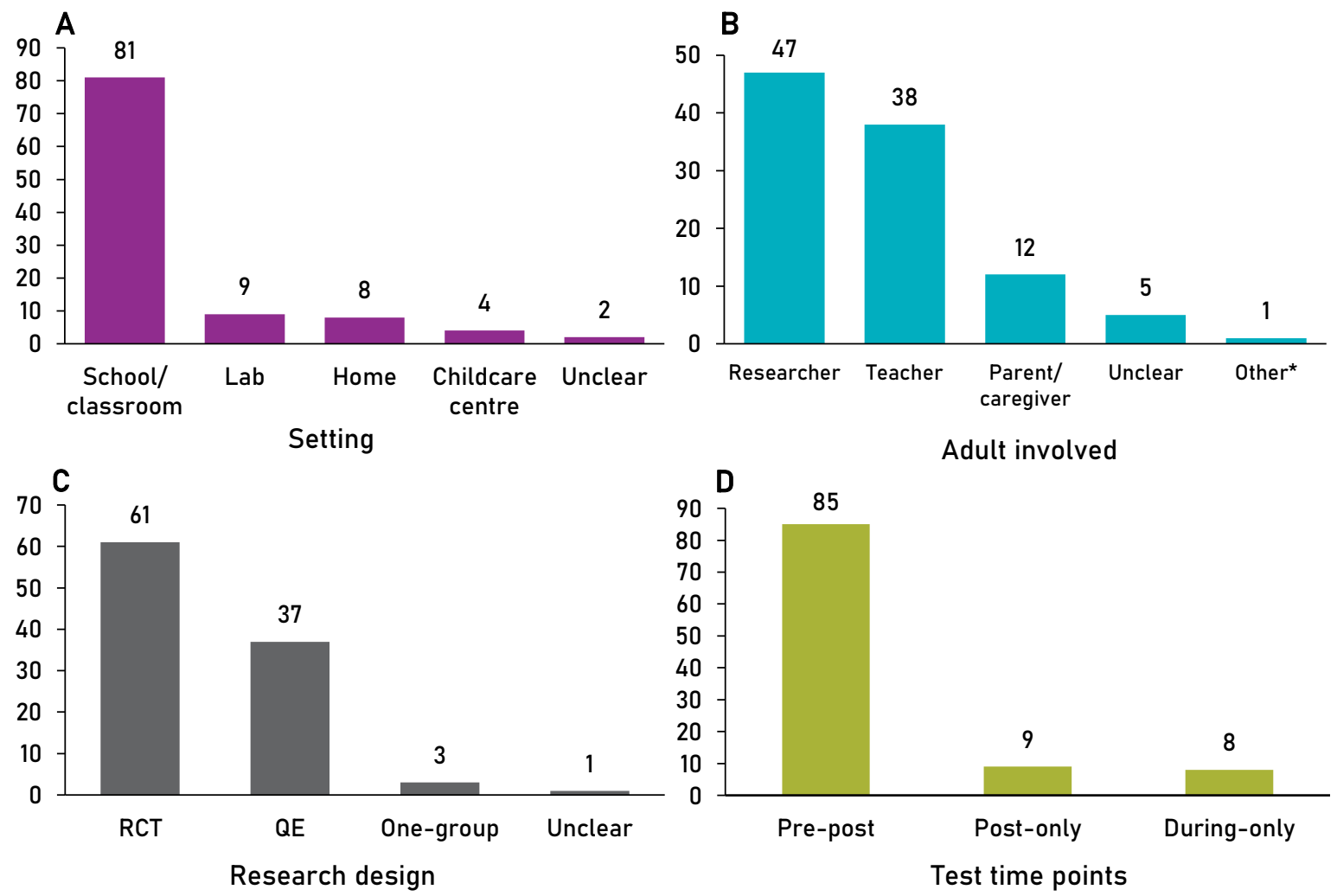

Figure 7. Distribution of included studies by (A) setting, (B) adult involved, (C) research design, and (D) test time points. The total $N$ varies due to overlap in some categories (e.g., an intervention conducted in a school and home setting with a teacher and caregiver). Other $=$ school psychologist. RCT $=$ randomised control trial. $\mathrm{QE}=$ quasi-experimental. 


\subsubsection{Level of instructional guidance \& play}

The current review considered similarities and differences in how PM-based interventions were delivered, according to four categories along the spectrum of learning through play (free play, guided play, games, and direct instruction; see Figure 2) ${ }^{9}$. For a broad overview of the types of instructional guidance used across the included studies, see Figure 8. Most studies involved guided play, games with rules, and direct instruction. Free play was sometimes used as a comparator, but not the main intervention condition. Each type of guidance is considered in turn below.

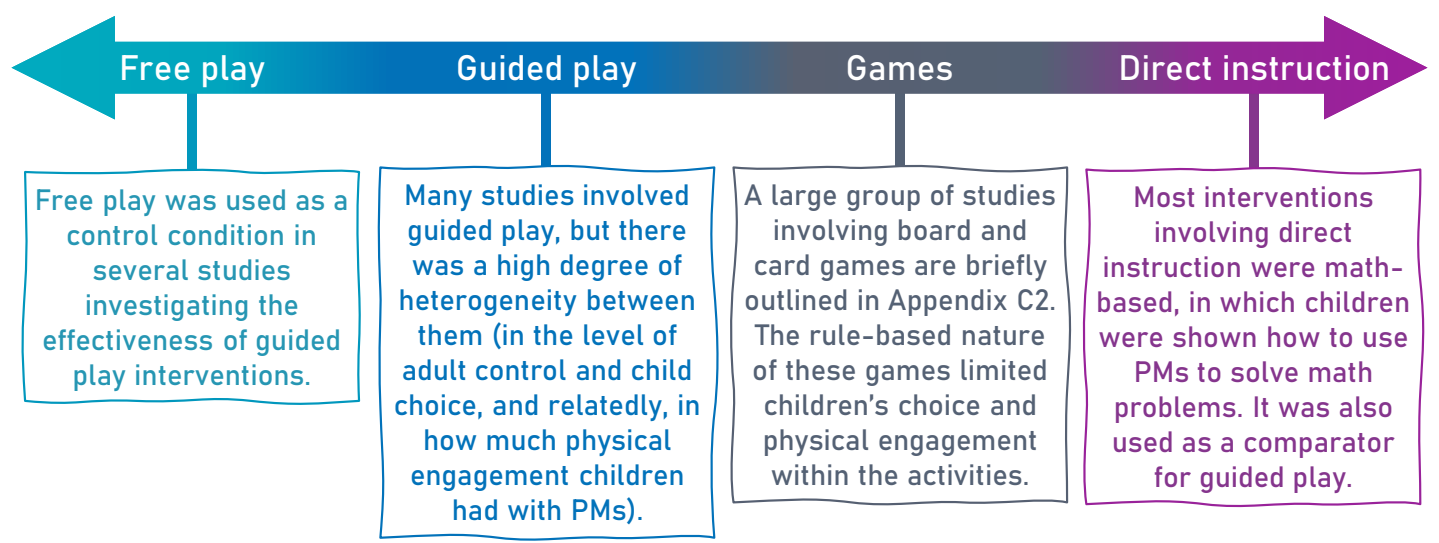

Figure 8. An overview of the level of instructional guidance across the included review studies according to the spectrum of learning through play.

\subsubsection{Free play}

None of the included studies implemented free play with PMs as the main intervention of interest, though some had limited opportunities for free play among other structured activities [e.g., Borriello]. Free play was used as a control condition for studies evaluating the impact of guided play interventions involving block building [e.g., Ferrara], math PMs [e.g., Eason], reading/literacy-based PMs [Toub], science-based PMs [van Schijndel], and toys [Clearfield]. For example, one study compared the impact of guided play in a sandpit - during which a teacher encouraged young children's exploration and experimentation with various materials - to free play with the same materials [van Schijndel]. Overall, most studies reported that guided play with PMs was more effective than free play with the same or similar materials for children's visual-spatial, language, and exploratory play outcomes [Borriello; Casey 2008a; Eason; Ferrara; Fisher 2011b, 2013; Toub; van Schijndel].

\subsubsection{Guided play}

Overall, a large proportion of studies involved PM-based guided play interventions - that is, interventions in which children engaged in play-based activities, were afforded some degree of freedom/choice, and were guided or supported by an adult. Some studies were explicitly described as being guided play [e.g., Cavanaugh; Han; Jemutai] or used some variation of the term such as

\footnotetext{
9 Though considered across the review studies, the type of instruction was not systematically coded during data extraction due to the subjective nature of doing so (only a single researcher conducted the data extraction).
} 
scaffold, assist, or support in relation to adult involvement in playful tasks [e.g., Bennett]. In other studies, the term guided play was not ascribed to interventions, but activities encompassed core features of guided play [e.g., Burte; Li; Pirrone; Taylor].

Notably, most studies in the block building category involved guided play ( $\sim 80 \%)$. Over the past decade, block play interventions have garnered attention due to associations between children's spatial play and their spatial and math ability (see Section 4.3.1 for an overview). Consequently, there has been a proliferation of structured block play during which children build a target structure from a model or illustration. Though structured versus free play with blocks limits children's choice and creativity within building activities (and therefore potentially being deemed less playful), it is theorised that building predefined structures specifically encourages children to mentally visualize and transform objects (e.g., segmenting objects into parts and combining into a whole, transforming a 2D image into a 3D structure) (Newman et al., 2021).

Across the interventions involving guided play, the amount of child choice and adult guidance - two core features of guided play - varied substantially (for an overview of the key features of guided play, see Section 2.2.2). In some studies, authors acknowledged the value of learning through play, and delivered interventions that encouraged child-led playful activities and sensitive adult guidance (using techniques such as modelling, setting a challenge, and adjusting to the needs of individual children; [e.g., Cavanaugh; Toub; van Schijndel]). For example, in a reading/literacy intervention, children's play with story-relevant PMs was scaffolded by an adult who followed their lead, engaged in co-play, asked open-ended questions, and incorporated, defined, and modelled the use of target words at naturally-occurring moments [Toub]. In contrast, while other authors may have acknowledged the value of play and adult guidance in their theoretical frameworks, the intervention activities were more constrained and afforded children relatively less freedom (e.g., provided fewer opportunities for children to take the lead and/or involved adult guidance that was not tailored to individual children). For example, in some studies children constructed predefined structures using images that illustrated each major step of the build [e.g., Boyle; Ferarra; Sawyer], and in others the adult followed a script with set questions [e.g., Eason; Fisher 2001a, b]. Therefore, when considering the spectrum of learning through play (see Figure 8), some guided play interventions were more like free play than direct instruction, or vice versa.

\subsubsection{Games}

A substantial number of interventions identified in the review involved games with rules. Studies involving board and cards games $(n=25)$ are summarised briefly in Table E2 (see Appendix E, section 7.5) and are not discussed in detail10. Most involved linear number boardgames that targeted children's counting skills [e.g., Siegler; Whyte], or other math/numeracy-related games for practicing skills such as magnitude comparison [Ramani 2020]. Due to the rule-based nature of these games, there were limited opportunities for child choice within the activities. Furthermore, the amount of

\footnotetext{
10 There are also several studies involving games with rules in other study groupings, such as block building [Newman] and puzzles [Lu]. The distinction between highly structured guided play activities (that permit relatively low levels of child choice) and games with rules can be somewhat arbitrary and subjective. Note that studies involving boardgames and card games are not discussed in detail due to the low levels of physical manipulation that children engaged in with the intervention materials.
} 
physical engagement was restricted as children could typically only perform simple actions such as moving a counter along a number line or turning over a card.

\subsubsection{Direct instruction}

Didactic teaching methods were used as the main instructional technique in numerous PM-based interventions, especially those involving math-based PMs [e.g., Ermakova; Fujimura; Martin; MoyerPackenham; Watchorn]. In these studies, children were typically shown how to use math PMs such as fraction bars, counters, cubes, or coins by an adult, before using them to solve math problems. PMs are well-established teaching aids in math classrooms as they facilitate children's learning of abstract math concepts through developmentally appropriate hands-on experiences. Therefore, it is unsurprising that many of the math-based PM interventions involved traditional didactic teaching approaches ${ }^{11}$ (for existing reviews regarding the efficacy of teaching math with PMs, see Carbonneau et al., 2013; Sowell, 1989). Didactic instruction was also used as the main instructional method in other study categories: most interventions involving paper folding involved direct instruction - with each folding step being modelled by an adult [e.g., Cakmak; Yuzawa], and some reading/literacy-, puzzle-, and science-based interventions involved children performing more prescribed actions with materials [e.g., Biazak; Cobb; Dang; Lane; Olkun; Lazonder; Zacharia]. For example, in two sciencebased studies, children simply dropped objects from different heights [Lazonder] or placed items on a balance beam [Zacharia]. Finally, as with free play, direct instruction (e.g., business-as-usual, traditional teaching) was often used as a control condition for play-based approaches [e.g., Bennett; Bulunuz; Casey 2008b; Cavanaugh; Eason; Fisher 2011a, 2011b, 2013; Han; Pirrone; Toub; WillsonQuayle].

${ }^{11}$ But not exclusively; there were also play-based interventions involving math PMs [e.g., Bennett; Eason; Fisher 2011a, 2011b, 2013; Hawes; Sophian]. 


\subsection{Review of interventions involving physical manipulatives (PMs)}

The $102^{12}$ studies included in the review were diverse. A categorisation scheme was developed to broadly group similar interventions by the types of materials and activities involved ${ }^{13}$. Seven categories relevant to the current review aims were identified, and are ordered as follows:

1. Building blocks $(n=19)$

2. Math-based PMs $(n=26)$

3. Reading/literacy PMs $(n=8)$

4. Science-based PMs $(n=9)$

5. Paper folding $(n=7)$

6. Puzzles and Tangrams $(n=6)$

7. Other: basic toy play/exploration $(n=2)$ and boardgames and card games $(n=25)$

In the following sub-sections, categories 1-6 are reviewed in turn using a consistent framework. The interventions are discussed, with similarities and differences relating to numerous factors being considered (e.g., the type of instruction, the level of play, materials used, activities, level of physical engagement with PMs). Next, evidence related to effectiveness is summarised with consideration of study methodologies (further details regarding the results of individual studies are provided in Appendix D (section 7.4). Gaps in the research are also considered and tables summarising study characteristics are provided.

Studies included in the final category - Other - are briefly summarised in Appendix E (section 7.5) but are not discussed in detail in the main report ${ }^{14}$. An additional seven studies were identified in the search but were excluded due to limited information in the reports despite being potentially relevant for inclusion; citations for these studies are provided in Appendix G (section 7.7).

\footnotetext{
${ }^{12}$ For duplicate studies (for example, a PhD thesis and a published journal article reporting on the same data), only the later source is included (though, the other document may have been consulted for additional information during data extraction).

${ }^{13}$ Studies that could fit into more than one category (for example, a study compared a block building intervention to a board ga me control group), the intervention that was considered the main intervention in the study was used for categorisation. For example, [Newman] was included in the block building category, as block building was the main intervention, and the board game activity was used as the control. 14 These studies were deemed less relevant for the current review due to the limited amount of physical manipulation that boardgames and card games afford.
} 


\subsubsection{Block building $(n=19)$}

\section{KEY FINDINGS}

- Children typically built structures based on pre-designed models/illustrations using bricks or blocks.

- Most interventions used a form of guided play.

- Benefits were reported to children's spatial talk, spatial ability, \& math skills, though some studies reported null effects in these domains.

\section{Overview}

All the studies discussed in this section involved children engaging in some degree of building/constructing with blocks or bricks (henceforth simply referred to as blocks) during the intervention activities. Across and within studies both the degree of physical engagement with the manipulative materials and the level of freedom afforded to children during the intervention activities differed. The interventions were overwhelmingly conducted in high-income contexts $(n=$ 15), with none taking place in low-income countries.

\begin{tabular}{|ll|}
\hline & KEY INTERVENTION FEATURES \\
Location & USA (11), South Africa* (2), China (1), Italy (1), Kenya ${ }^{*}(1)$, \\
& Netherlands (1), Papua New Guinea (1), Taiwan (1), Sweden (1) \\
Setting & School/classroom (13), research lab (3), home (2), unclear (1) \\
Adult involved & Researcher (9), teacher/TA (5), parent/caregiver (4), unclear (1) \\
Main outcome domains & Visual-spatial (7), executive function (4), spatial language (3), \\
& social-emotional (4), visual perception (3), math/numeracy (3), \\
& fluid intelligence (2), play-based (1), and other ${ }^{\star \star}(4)$
\end{tabular}

Note. $n$ s in parentheses corresponds to $n$ studies. ${ }^{*}$ One study included two samples for different countries: one from South Africa and one from Kenya, which were analysed separately. ${ }^{* *}$ Including brain imaging, logical thinking, creative personality, and drawing skills.

\section{Interventions}

See Table 1 for a summary of study characteristics. Overall, children engaged in high levels of physical manipulation with materials ${ }^{15}$ - all the interventions involved a construction/building activity. In many cases, construction play was a core focus of the intervention. In some, children engaged in semistructured block play in which they built a target structure using step-by-step instructional images or a pre-built model as a guide [e.g., Ferrara; Goldstein; Newman; Sawyer; Schmitt]. One study afforded children more creativity in terms of what they built: the teacher asked pupils to imagine something (e.g., their favourite number), build it from LEGO bricks, and then draw it [Pirrone]. In another paper, the block building activity was embedded in a storytelling context, whereby children were helping 'King' and 'Queen' characters (represented by puppets) to rebuild their castle [Casey 2008a]. In several interventions, while children had some opportunities for construction play, it was

\footnotetext{
${ }^{15}$ Relative to the other study groupings.
} 
part of various other activities like sorting, stacking, making patterns, putting blocks in a box, and manually exploring blocks [Bugos; Christakis; Schröder; Vander Heyden]. In two studies, while children did engage in construction block play, they also used the materials for learning math operations such as addition [Pirrone; Simoncini] ${ }^{16}$.

Two studies involved the Six Bricks programme - an intervention involving various guided play activities that afforded children opportunities to direct their own learning - in Kenya and South Africa [Brey; Jemutai]. Children were each provided with their own set of six DUPLO bricks (each one a different colour), which were readily available to them during various classroom activities throughout the school day. Teachers attended training on how to use the materials in lessons and were provided with a file describing numerous Six Bricks activities. For example, the teacher might demonstrate an activity, like building a shape, and then ask children to replicate what they had done before continuing to play (e.g., building their own shapes). Teachers were free to choose how and when to incorporate the materials and suggested activities into their classroom teaching, meaning the specific ways in which children engaged with the physical materials in these interventions were unclear. In both studies, the activities selected by the researchers focused on developing visual perception or visual-spatial ability but were not explicitly described. A third paper, which examined the impact of Six Bricks in Taiwan, reported limited information about the implementation and delivery of the program: the setting (e.g., classroom or home), adult involved (e.g., teacher or experimenter), and model of delivery (e.g., one-on-one or classroom) remain unclear [Harn]. Children engaged in various collaborative activities like building a tower or a bridge together, but unlike the other Six Bricks papers in this review, the intervention focused on fostering positive emotions rather than visual and/or spatial abilities.

In some studies, the specific activities that children engaged in were prescriptive and remained constant for all children in the intervention group [e.g., Borriello; Sawyer]. For example, children played a game (Blocks Rock!) in which they had to build a structure illustrated on a card as fast as they could [Newman], or constructed target structures using images that illustrated each major step of the build, with guidance from an adult [Sawyer]. In other studies, the specific activities and the ways in which children engaged with the PMs were less defined, as they focused on providing teachers or parents with training and/or activity suggestions. In some, parents and caregivers were provided with blocks and recommended activities to engage their child in at home [Christakis; Schröder], and in two of the Six Bricks studies, teachers attended training on how to implement guided block play activities into their classrooms [Brey; Jemutai]. Similarly, teachers in [Simoncini] attended workshops and then chose how to incorporate PMs for play-based learning. Therefore, children's playful learning experiences and the types of PM activities they engaged in likely varied within these studies.

The level of adult guidance and child autonomy also differed within and across studies. Many could be described as guided play interventions: those in which a playful learning activity was scaffolded by an adult, and afforded children some autonomy/freedom to explore within the activity. Some were explicitly described as guided play interventions or included some variation of the term, such as scaffold, support, or assistance, in relation to adult involvement in play-based tasks [Borriello; Brey;

\footnotetext{
${ }^{16}$ Therefore, overlapping with studies in the math PMs section (4.3.2).
} 
Casey, 2008a; Ferrara; Goldstein; Jemutai; Sawyer; Tian; Vander Heyden; Willson-Quayle]. For example, one study compared adult-scaffolded play in which children built a LEGO house with an experimenter who facilitated the activity (by helping, asking questions, and verbalising some of their actions), to other conditions that were entirely adult-directed or child-centred (i.e., free play) [Willson-Quayle]. In other studies, the term guided play was not ascribed to the playful learning experiences in the interventions, yet they could still meet the criteria for guided play. For example, children in two studies took part in adult-initiated block building activities in which they had some choice in how or what they made and were guided by prompts from an adult [Pirrone; Schmitt]. In other studies, parent-child dyads engaged in joint block play; the caregiver encouraged their child to actively participate and explore blocks [Schröder] or encouraged their child's spatial thinking and language by talking about spatial relations during play [Borriello].

\section{Evidence relating to effectiveness}

Findings are discussed in relation to specific outcome domains, including spatial language, visualspatial, visual perception, executive function, fluid intelligence, math/numeracy, and socialemotional learning, and are discussed in the context of methodological variations across studies. See section Appendix D (section 7.4.1) for an overview of main outcome domains and findings organised by study.

There is evidence that parent-guided block play increases children's spatial language: children in guided block play groups produced more spatial talk than controls who engaged in free play with blocks [Borriello; Ferrara]. In one of these studies, the intervention also resulted in lower levels of pretend play [Borriello]. Although the level of adult guidance and child autonomy differed between groups in these studies, block play interventions were not contrasted with non-PM conditions. Another study found that while overall, there were no group differences on language acquisition scores between children who received building blocks at home and those who did not, there were benefits specifically for children from low- and middle-income homes who received blocks [Christakis].

Evidence for benefits to visual-spatial measures is inconclusive due to methodological inconsistencies across studies. One study found that structured block building activities improved spatial reasoning relative to regular teaching plus some free play with blocks [Casey, 2008a] or a control drawing intervention [Tian], and while another also found that guided block building improved children's spatial reasoning, the study lacked a comparison group [Boyle]. Scaffolded and adult-directed block play increased children's block building skills, whereas child-centred free-play did not [Willson-Quayle]. Gains in mental imagery were observed for a block building versus regular teaching control group, however the authors did not examine baseline variations between the two groups on this measure [Pirrone]. The impact of block play interventions on visual-spatial transformation ability is mixed: there were no group differences in performance gains on mental folding [Vander Heyden] or mental rotation [Casey, 2008a; Newman; Vander Heyden]. Despite the lack of group differences in gain scores, [Newman] conducted follow-up within-group analyses revealing gains following a block play but not control boardgame (Scrabble) intervention. Another study reported benefits to spatial perspective taking after a spatial training intervention (involving various activities such as block building and making a marble run) relative to controls [Vander Heyden]. However, the analysis did not control for the potential impact of baseline variations at 
outcome. Therefore, caution must be taken when interpreting these findings. Interestingly, [Newman] also found that block play was associated with increased functional brain activity in areas implicated in spatial processing.

There is some limited evidence that block play interventions benefit children's visual perception. Two studies reported benefits of the Six Bricks programme to visual perception relative to passive controls [Brey; Jemutai]. However, in one study the statistical models used were unclear [Brey] and in the other, positive effects were only found when the authors used an adjusted $\alpha(p<.10$; the effects were not sustained when using $\alpha \quad p<.05$ ). Another study found that block play, relative to book reading, benefited children's visual form sensitivity, but not numerosity discrimination [Schröder].

There was no clear evidence that block-building interventions improved executive function measures of inhibition, attention, cognitive flexibility, or global executive functioning [Bugos; Christakis; Schmitt; Schröder]. In fact, performance on an inhibition task worsened following LEGO training but not music training [Bugos]. Two studies examined children's fluid intelligence and found contrasting results: in one, the Six Bricks intervention was no better than a passive control condition for improving non-verbal reasoning [Brey], while in another, children who engaged in teacherguided block building showed larger gains than those who only attended regular math classes.

Mixed results were also reported for math and numeracy outcomes. One study reported that guided block play improved math scores relative to regular teaching [Pirrone]. Another found selective benefits: overall performance on a math assessment was better for children who received various blocks and bricks, compared to those who did not; but intervention children outperformed controls on only two out of five subtests (backward number sequence and early arithmetic strategies, but not subitising, number identification, or forward number sequence; [Simoncini]). This study is also limited by lacking baseline data for the outcomes. Although children in these interventions engaged in some degree of construction play with blocks, they also used the PMs during math instruction (e.g., to experience math operations like addition). Therefore, observed benefits to math outcomes could be mediated by the math content in the interventions rather than block building play ${ }^{17}$. Finally, another study failed to observe benefits to several math outcomes, including numeracy, shape recognition, or math language, though some positive findings were mediated by parental education level [Schmitt].

Four studies considered the effects of block play on social-emotional outcomes. One reported no impact on various measures (e.g., theory of mind, social interaction; [Goldstein]), another demonstrated that none of three block play conditions (each with varying degrees of adult control and child autonomy) affected happiness scores but did increase negative emotions overall [WillsonQuayle], and no clear conclusions could be drawn from a third study involving the Six Bricks intervention (see Appendix D, section 7.4.1, for more details; [Harn]). A final study reported that block play, relative to a drawing control condition, enhanced children's theory of mind scores; an effect that was potentially mediated by improvements in their visual perspective taking [Tian].

\footnotetext{
${ }^{17}$ Note that other reports included in the wider review have been categorised as interventions involving math PMs. There is some overlap between these two studies that involved math learning with blocks (Pirrone et al., 2018; Simoncini et al., 2020) and studies included in the math PMs category.
} 
It is difficult to disentangle whether any observed positive effects are due to the physical nature of children engaging in block play. This is due to differences in the comparison groups across the studies. Positive effects in studies employing passive (no-intervention) control groups may be driven by expectancy, motivational, and/or Hawthorne effects. Though, some studies compared block building to control conditions that did not involve PMs (i.e., story time, book reading, pretend play; [Sawyer; Goldstein; Schröder]). In another study, although children in all groups engaged in block play, the degree of physical engagement differed between conditions [Willson-Quayle]. Children in the adult-scaffolded block play and free-play groups had more freedom to create and build with the materials than those in an adult-directed group in which the researcher gave explicit/direct building instructions and commands. The degree of physical engagement appears to be associated with the level of adult guidance: less adult instruction or guidance means children had more freedom to physically engage with the materials.

\section{Summary}

Key findings

To summarise, there was some evidence that block-building activities benefit children's learning outcomes across several areas of functioning, including spatial language, spatial reasoning, and math. However, findings were mixed and inconclusive for all domains. Overall, there was no clear evidence that block-building interventions consistently benefit children's outcomes, at least in part due to methodological inconsistencies across studies.

Key gaps \& recommendations for future research

- Overall, the field is lacking high-quality studies with robust research designs. There was also a clear dearth of research in low-income contexts - with over half of the block building interventions taking place in the USA, thus limiting the generalisability of study findings. Two studies were conducted in lower-middle-income countries (Kenya and Papua New Guinea) but despite both reporting positive effects, the studies are limited by methodological shortcomings. There is a pressing need for further research in low-income contexts especially research that is methodologically rigorous. As will be seen in subsequent sections of this report - this general theme emerged across the review literature.

- Further systematic investigations designed to isolate the critical intervention components that mediate improvements are needed. Currently, it is difficult to disentangle the intervention features that are most beneficial, due to numerous factors being manipulated across and within studies (e.g., instructional guidance, physical engagement, materials, etc.).

- Many (but not all) studies focused on semi-structured block building activities in which children replicated a predefined structure. Future research could focus on developing interventions that provide children with more freedom to express their creativity and inventiveness when engaging in block building activities - allowing them to imagine, design, and build their own creations - whilst being scaffolded by an adult.

- Finally, of note is that all three studies that examined the impact of parent-child block play on language outcomes found positive effects, and further research could establish if these benefits can be replicated in other contexts (as all were conducted in the USA) and/or learning domains. 
PEDAL Report: PM scoping review

Table 1. Characteristics of studies in the block building category $(n=17)$.

\begin{tabular}{|c|c|c|c|c|c|c|c|c|c|c|c|c|}
\hline ID & Location & $\begin{array}{c}\text { Sample } \\
N\end{array}$ & Age & $\begin{array}{l}\text { Research } \\
\text { design }\end{array}$ & Setting & $\begin{array}{l}\text { Model of } \\
\text { delivery }\end{array}$ & $\begin{array}{c}\text { Adult } \\
\text { involved }\end{array}$ & $N$ sessions & Materials & Intervention & Comparison(s) & $\begin{array}{c}\text { Main outcome } \\
\text { domains }\end{array}$ \\
\hline $\begin{array}{l}\text { Borriello } \\
2018\end{array}$ & USA & 41 & $4-6$ & $\begin{array}{l}\text { QE, } \\
\text { during- } \\
\text { only }\end{array}$ & $\mathrm{Lab}$ & Individual & $\begin{array}{l}\text { Parent/ } \\
\text { caregiver }\end{array}$ & 1 & LEGO, puzzle & $\begin{array}{l}\text { Puzzle activity, free \& structured } \\
\text { play with LEGO, mothers informed } \\
\text { on value of promoting spatial skills }\end{array}$ & $\begin{array}{l}\text { Same, but mothers } \\
\text { not told about } \\
\text { spatial skills }\end{array}$ & $\begin{array}{l}\text { Literacy/ } \\
\text { language, play- } \\
\text { based }\end{array}$ \\
\hline $\begin{array}{l}\text { Boyle } \\
2017\end{array}$ & USA & 29 & $4-6$ & $\begin{array}{l}\text { One } \\
\text { group, } \\
\text { pre-post }\end{array}$ & $\begin{array}{l}\text { School/ } \\
\text { classroom }\end{array}$ & Group & Researcher & $>20$ & Building blocks & $\begin{array}{l}\text { Block building simple \& complex } \\
\text { predefined structures (e.g., tower, } \\
\text { castle, giraffe); guided by images }\end{array}$ & $\begin{array}{l}\text { N/A (no control } \\
\text { group) }\end{array}$ & Visual-spatial \\
\hline $\begin{array}{l}\text { Brey } \\
2017\end{array}$ & South Africa & 443 & $7-9$ & $\begin{array}{l}\text { QE, pre- } \\
\text { post }\end{array}$ & $\begin{array}{l}\text { School/ } \\
\text { classroom }\end{array}$ & Class & Teacher & $>20$ & DUPLO & Six Bricks (various activities) & No-intervention & $\begin{array}{l}\text { Visual } \\
\text { (perception), } \\
\text { fluid intelligence }\end{array}$ \\
\hline $\begin{array}{l}\text { Bugos } \\
2017\end{array}$ & USA & $34^{\mathrm{a}}$ & $4-6$ & $\begin{array}{l}\text { RCT, pre- } \\
\text { post }\end{array}$ & $\begin{array}{l}\text { School/ } \\
\text { classroom }\end{array}$ & Group & Researcher & $11-20$ & LEGO & $\begin{array}{l}\text { Structured block play, sorting, } \\
\text { creative building }\end{array}$ & Music training & $\begin{array}{l}\text { Executive } \\
\text { function }\end{array}$ \\
\hline $\begin{array}{l}\text { Casey } \\
2008 a\end{array}$ & USA & 100 & $4-6$ & $\begin{array}{l}\text { QE, pre- } \\
\text { post }\end{array}$ & $\begin{array}{l}\text { School/ } \\
\text { Classroom }\end{array}$ & $\begin{array}{l}\text { Class/ } \\
\text { group }\end{array}$ & Teacher & $6-10$ & Building blocks & $\begin{array}{l}\text { Structured block building with } \\
\text { story/puppets. Children helped } \\
\text { characters in the story to re-build } \\
\text { a 'castle' with pre-defined features }\end{array}$ & $\begin{array}{l}\text { 1. Structured } \\
\text { building, no story } \\
\text { 2. Regular math } \\
\text { class \& free play } \\
\text { with blocks }\end{array}$ & Visual-spatial \\
\hline $\begin{array}{l}\text { Christakis } \\
2007\end{array}$ & USA & 140 & $0-3$ & $\begin{array}{c}\text { RCT, } \\
\text { post-only }\end{array}$ & Home & Individual & $\begin{array}{l}\text { Parent/ } \\
\text { caregiver }\end{array}$ & Unclear b & Building blocks & $\begin{array}{c}\text { Parents given blocks \& activities to } \\
\text { use at home (specific activities } \\
\text { unclear) }\end{array}$ & $\begin{array}{l}\text { Waitlist control } \\
\text { (distribution of } \\
\text { bricks at end of } \\
\text { study) }\end{array}$ & $\begin{array}{l}\text { Spatial } \\
\text { language, } \\
\text { executive } \\
\text { function }\end{array}$ \\
\hline $\begin{array}{c}\text { Ferrara } \\
2011\end{array}$ & USA & 72 & $4-6$ & $\begin{array}{l}\text { RCT, } \\
\text { during- } \\
\text { only }\end{array}$ & Lab & Individual & $\begin{array}{l}\text { Parent/ } \\
\text { caregiver }\end{array}$ & 1 & $\begin{array}{l}\text { Building blocks, } \\
\text { figurines }\end{array}$ & $\begin{array}{l}\text { Parent-guided block play: dyads } \\
\text { used photos that guided building } \\
\text { (e.g., garage, helipad) \& placement } \\
\text { of figurines }\end{array}$ & $\begin{array}{l}\text { 1. Free play with } \\
\text { blocks } \\
\text { 2. Play with pre- } \\
\text { made structures }\end{array}$ & Spatial language \\
\hline $\begin{array}{l}\text { Goldstein } \\
2018\end{array}$ & USA & $79 \mathrm{c}$ & $4-6$ & $\begin{array}{l}\text { RCT, pre- } \\
\text { post }\end{array}$ & $\begin{array}{l}\text { School/ } \\
\text { Classroom }\end{array}$ & Group & Researcher & $>20$ & Building blocks & $\begin{array}{l}\text { Block building simple \& complex } \\
\text { pre-defined structures (e.g., tower, } \\
\text { castle, giraffe); guided by images }\end{array}$ & $\begin{array}{l}\text { 1. Dramatic } \\
\text { pretend play } \\
\text { 2. Story time }\end{array}$ & Social-emotional \\
\hline $\begin{array}{l}\text { Harn } \\
2019\end{array}$ & Taiwan & 24 & $\begin{array}{l}10- \\
12\end{array}$ & $\begin{array}{l}\text { RCT, pre- } \\
\text { post }\end{array}$ & Unclear & Unclear & Unclear & Unclear & DUPLO & $\begin{array}{l}\text { Six Bricks: various activities, } \\
\text { cooperative building, self- } \\
\text { reflection, thinking about } \\
\text { 'happiness' }\end{array}$ & No-intervention & Social-emotional \\
\hline $\begin{array}{l}\text { Jemutai } \\
2019\end{array}$ & $\begin{array}{l}\text { South Africa } \\
\text { \& Kenya }\end{array}$ & 77 & $4-6$ & $\begin{array}{l}\text { QE, pre- } \\
\text { post }\end{array}$ & $\begin{array}{l}\text { School/ } \\
\text { classroom }\end{array}$ & $\begin{array}{l}\text { Class/ } \\
\text { group }\end{array}$ & Teacher & $>20$ & DUPLO & $\begin{array}{c}\text { Six Bricks: activities targeting } \\
\text { visual skills }\end{array}$ & No-intervention & $\begin{array}{c}\text { Visual } \\
\text { (perception) }\end{array}$ \\
\hline $\begin{array}{l}\text { Newman } \\
2016\end{array}$ & USA & 28 & $7-9$ & $\begin{array}{l}\text { QE, pre- } \\
\text { post }\end{array}$ & Lab & $\begin{array}{l}\text { Individual/ } \\
\text { pairs }\end{array}$ & Researcher & $2-5$ & $\begin{array}{l}\text { Building blocks, } \\
\text { card game }\end{array}$ & $\begin{array}{l}\text { Commercial block play game } \\
\text { (Blocks Rock!). Children built } \\
\text { target structures as fast as they } \\
\text { could to win the game }\end{array}$ & $\begin{array}{l}\text { Commercial word } \\
\text { boardgame } \\
\text { (Scrabble) }\end{array}$ & Visual-spatial \\
\hline $\begin{array}{l}\text { Pirrone } \\
2018\end{array}$ & Italy & 33 & $4-6$ & $\begin{array}{l}\text { RCT, pre- } \\
\text { post }\end{array}$ & $\begin{array}{l}\text { School/ } \\
\text { classroom }\end{array}$ & Class & Teacher & $>20$ & LEGO & $\begin{array}{l}\text { Imagining \& building LEGO } \\
\text { creation, using LEGO to learn math } \\
\text { operations }{ }^{\mathrm{d}}\end{array}$ & $\begin{array}{l}\text { Business-as-usual } \\
\text { (regular math } \\
\text { classes) }\end{array}$ & $\begin{array}{l}\text { Math/numeracy, } \\
\text { visual-spatial, } \\
\text { fluid intelligence }\end{array}$ \\
\hline
\end{tabular}




\section{PEDAL Report: PM scoping review}

\begin{tabular}{|c|c|c|c|c|c|c|c|c|c|c|c|c|}
\hline $\begin{array}{l}\text { Sawyer } \\
2019\end{array}$ & USA & 77 & $4-6$ & $\begin{array}{l}\text { RCT, } \\
\text { during- } \\
\text { only }\end{array}$ & $\begin{array}{l}\text { School/ } \\
\text { classroom }\end{array}$ & Group & Researcher & $>20$ & $\begin{array}{l}\text { DUPLO or } \\
\text { MegaBlocks }\end{array}$ & $\begin{array}{l}\text { Structured block play with adult } \\
\text { guidance }\end{array}$ & $\begin{array}{l}\text { 1. Storytime } \\
\text { 2. Pretend play }\end{array}$ & $\begin{array}{l}\text { Other (drawing } \\
\text { skills) }\end{array}$ \\
\hline $\begin{array}{l}\text { Schmitt } \\
2018\end{array}$ & USA & 59 & $4-6$ & $\begin{array}{l}\text { RCT, pre- } \\
\text { post }\end{array}$ & $\begin{array}{l}\text { School/ } \\
\text { classroom }\end{array}$ & Group & Researcher & $11-20$ & Building blocks & $\begin{array}{l}\text { Semi-structured block play, } \\
\text { children guided by prompts \& } \\
\text { shown pictures of structures }\end{array}$ & Business-as-usual & $\begin{array}{l}\text { Math/numeracy, } \\
\text { executive } \\
\text { function }\end{array}$ \\
\hline $\begin{array}{l}\text { Schröder } \\
2019\end{array}$ & Sweden & 58 & $0-3$ & $\begin{array}{l}\text { RCT, pre- } \\
\text { post }\end{array}$ & Home & Individual & $\begin{array}{l}\text { Parent/ } \\
\text { caregiver }\end{array}$ & $>20$ & Building blocks & $\begin{array}{l}\text { Caregivers given blocks \& } \\
\text { activities to encourage manual } \\
\text { exploration (e.g., put blocks in box, } \\
\text { build tower, knock tower down) }\end{array}$ & Book reading & $\begin{array}{l}\text { Executive } \\
\text { function, visual } \\
\text { (perception) }\end{array}$ \\
\hline $\begin{array}{l}\text { Simoncini } \\
2020\end{array}$ & $\begin{array}{c}\text { Papua New } \\
\text { Guinea }\end{array}$ & 45 & $4-6$ & $\begin{array}{l}\text { QE, post- } \\
\text { only }\end{array}$ & $\begin{array}{l}\text { School/ } \\
\text { classroom }\end{array}$ & Class & Teacher & $>20$ & $\begin{array}{c}\text { DUPLO, } \\
\text { MegaBlocks, } \\
\text { wooden blocks }\end{array}$ & $\begin{array}{c}\text { Teachers received blocks (for } \\
\text { math/numeracy learning, also } \\
\text { some building/free play) e }\end{array}$ & $\begin{array}{l}\text { Business-as-usual } \\
\text { (no blocks) }\end{array}$ & $\begin{array}{l}\text { Math/ } \\
\text { numeracy }\end{array}$ \\
\hline $\begin{array}{l}\text { Tian } \\
2021\end{array}$ & China & $46^{\mathrm{f}}$ & $0-3$ & $\begin{array}{l}\text { QE, pre- } \\
\text { post }\end{array}$ & $\begin{array}{l}\text { School/ } \\
\text { classroom }\end{array}$ & Group & Researcher & $6-10$ & Wooden blocks & $\begin{array}{l}\text { Structured block building: } \\
\text { recreating preassembled models }\end{array}$ & $\begin{array}{l}\text { Drawing } \\
\text { intervention }\end{array}$ & $\begin{array}{l}\text { Visual-spatial, } \\
\text { social-emotional }\end{array}$ \\
\hline $\begin{array}{l}\text { Vander } \\
\text { Heyden } \\
2017\end{array}$ & Netherlands & 140 & $7-9$ & $\begin{array}{l}\text { QE, pre- } \\
\text { post }\end{array}$ & $\begin{array}{l}\text { School/ } \\
\text { classroom }\end{array}$ & Group & Researcher & $2-5$ & $\begin{array}{l}\text { Wooden blocks, } \\
\text { other spatial toys } \\
\text { \& games (e.g., } \\
\text { marble runs) }\end{array}$ & $\begin{array}{l}\text { Spatial training: playful activities \& } \\
\text { commercial games (block building, } \\
\text { construction, object } \\
\text { transformation) }\end{array}$ & Business-as-usual & Visual-spatial \\
\hline $\begin{array}{l}\text { Willson- } \\
\text { Quayle } \\
2001\end{array}$ & USA & 61 & $4-6$ & $\begin{array}{l}\text { RCT, pre- } \\
\text { post }\end{array}$ & $\begin{array}{l}\text { School/ } \\
\text { classroom }\end{array}$ & Individual & Researcher & 1 & DUPLO & $\begin{array}{l}\text { Scaffolded block play: structured } \\
\text { LEGO building with adult guidance }\end{array}$ & $\begin{array}{l}\text { 1. Adult-directed } \\
\text { block play } \\
\text { 2. Free play }\end{array}$ & $\begin{array}{l}\text { Visual-spatial, } \\
\text { social-emotional }\end{array}$ \\
\hline
\end{tabular}

Note. For inclusion in the review, a threshold of $\leq 20$ was used for the child sample $N$ (based on $N$ included in analys

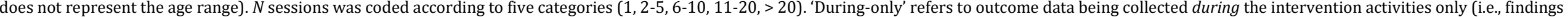

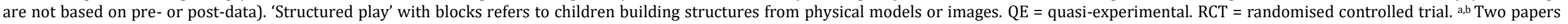

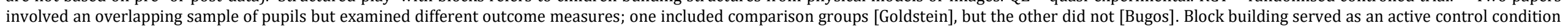

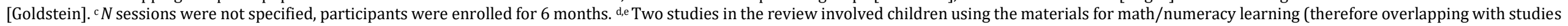

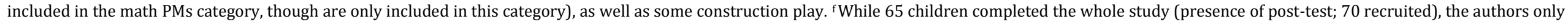
included 46 in the analyses (a sub-sample matched on several variables such as age, gender, and baseline block building ability. 


\subsubsection{Math-based PMs ( $\mathbf{n}=26)$}

\section{KEY FINDINGS}

- Numerous math concepts were targeted (e.g., fractions, measurement, counting, etc.) using a range of materials.

- Gains to children's math outcomes were reported following both didactic and playful approaches involving math PMs, though null or inconclusive results were also reported.

- Particularly promising positive effects were found in a lower-middleincome country following a robust \& large-scale effectiveness trial of a curriculum-based intervention.

- There was evidence that virtual manipulatives are just as effective, or better than, PMs for math learning.

\section{Overview}

Interventions involving PMs for math learning formed the largest category of studies in the review ${ }^{18}$. Consequently, there was an array of activities and math concepts being targeted across the interventions, including, but not limited to, tasks for learning geometry (e.g., shape sorting/matching, making shapes, exploring shapes via touch; [Fisher 2011a, 2011b, 2013; Gecu-Parmaksiz; Hull; Sophian; Thompson 2012]), fractions (e.g., manipulating fraction circles, pies, tiles, blocks [Aleid; Alshehri; Cramer; Marin; Moyer-Packenham]), measurement concepts such as area, volume, length, and weight (e.g., filling containers; [Dennis; Sophian]), probability (rolling dice, flipping coins [Taylor]), and proportional reasoning (with magnetic strips; [Fujimura]). Children also used counters, toys, sticks, and other small objects for counting, addition, sharing/dividing, and ordering/sorting by size [Bennett, Horan; Mattoon; Alghazo; Sophian; Starkey; Watchhorn]. Some interventions involved numerous activities (e.g., [Bennett; Hull; Sophian; Starkey]). Overall, children engaged in moderate levels of physical engagement - they did not use PMs in construction activities, but for the most part they physically interacted with PMs beyond simply holding/touching them

\begin{tabular}{|ll|}
\hline \multirow{3}{*}{ Location } & KEY INTERVENTION FEATURES \\
Setting & USA (19), Saudi Arabia (2), Belize (1), Canada (1), \\
& Japan (1), Turkey (1), United Arab Emirates (1) \\
& School/classroom (22), research lab (4), childcare \\
Adult involved & centre (1), home (2) * \\
Main outcome domains & Teacher (14), researcher (10), parent/caregiver (4)* \\
& Math/numeracy (23), spatial language (2), math- \\
& spatial (2), visual-spatial (2), receptive language (1)
\end{tabular}

*Two studies were conducted in both a school/classroom with a teacher and at home with a parent/caregiver [Sophian; Starkey], and another was conducted in a school/classroom and lab-based setting [Fisher 2013].

\footnotetext{
${ }^{18}$ Furthermore, studies in some of the other categories also involved using PMs for math learning (e.g., block building studies that also use PMs for math instruction or puzzle-based interventions for learning geometry).
} 
(with some exceptions). The degree of child choice and adult guidance also differed within and across studies. Geographically, studies were distributed across seven countries. Most took place in the USA, and only two were not conducted in a high-income country; namely Belize [Hull] and Turkey [GecuParmaksiz], which are lower-middle- and upper-middle-income countries, respectively.

\section{Interventions}

For a summary of the study characteristics, see Table 2. Children engaged in relatively more or less physical manipulation with materials across the studies, depending on the intervention activities. For example, children taking part in a geometry intervention participated in creative activities by combining/arranging PMs such as magnetic shapes, pentominoes, tiles, and cubes into more complex configurations and shapes (e.g., arranging shapes to make symmetrical patterns; [Hawes]). In other interventions, children simply touched or moved counters/small objects in counting activities (e.g., [Horan; Martin]). However, in some studies the level of physicality was difficult to determine. For example, in mixed-modality interventions the extent to which children engaged with PMs versus computer- or picture-based materials was unclear [Alghazo]. In another study with parent-child dyads, the authors describe a 'manual play' activity with tangible shapes, however it was unclear if or how children were encouraged to touch or manipulate the shapes themselves [Verdine]. The level of physical engagement also varied between conditions within some studies: several compared PMs and virtual or picture-based materials [Alshehri; Gecu-Parmaksiz; Martin; Mattoon; Mendiburo; Moyer-Packenham; Taylor; Verdine], or guided play to free play or direct instruction with PMs (in the latter, children could see but not touch the materials) [e.g., Eason, Fisher 2011a, 2011b, 2013].

While all the interventions involved math instruction to some extent, the particular math concept(s) being targeted varied. Children used PMs to help solve math problems about fractions [Alshehri; Aleid; Cramer; Eason; Martin; Mendiburo; Moyer-Packenham], probability [Taylor], measurement (e.g., volume, capacity, length) [Dennis; Sophian], counting [Alghazo; Bennett; Horan], geometry and spatial skills [Fisher 2011a, 2011b, 2013; Gecu-Parmaksiz 2018, 2019; Hawes; Thompson 2012; Verdine], proportional reasoning [Fujimura], and computation (e.g., addition, equivalent equations) [Ermakova; Mattoon; Watchhorn]. Some interventions targeted multiple concepts with the aim of enhancing general math achievement [Bennett; Hull; Sophian; Starkey]. Some math programmes were curriculum-based, meaning they included a range of materials and activities, took place over a several weeks or months during regular classroom instruction, and were integrated into, or implemented in lieu of, an existing math curriculum [Bennett; Cramer; Hawes; Hull; Sophian; Starkey; Thompson 2012].

Numerous interventions involving math-based PMs employed a traditional didactic teaching approach [e.g., Ermakova; Horan; Fujimura; Hull; Martin; Mattoon; Moyer-Packenham; Taylor; Watchorn] ${ }^{19}$. Though hands-on learning with PMs can arguably be more fun and engaging for young children, the math-based tasks offered children limited freedom and primarily required children to solve specific math problems after a demonstration from an adult. Furthermore, the traditional math

\footnotetext{
${ }^{19}$ However, note that in some cases it was difficult to determine the type of instruction (e.g., guided play versus direct instruction) due to papers reporting limited information regarding what children did in the intervention activities (how they used PMs) and what role the adult played (how they taught or supported children). Studies in the math-based PM category were often focused on the effectiveness of using concrete materials (e.g., as physical representations of abstract concepts) for math instruction rather than of the benefits of learning through play.
} 
PMs used in some studies were not playful and afforded limited opportunities for physical engagement; for example, children learned about fractions using fraction bars, tiles, and pies/circles [e.g., Aleid; Alshehri; Cramer; Martin; Moyer-Packenham]. While in some papers, the authors used terms such as scaffolded learning and adult guidance in their theoretical framework [e.g., Ermakova] or manipulated the level of adult guidance/child freedom across conditions [Horan], they were not play-based.

Some interventions employed a guided play approach to learning [Eason; Fisher 2011a, 2011b, 2013]. For example, several studies compared guided play with PMs to free play or didactic instruction [Eason, Fisher 2011b, 2013]. In these playful interventions, children learned about 'whole' and 'parts' by sharing segmented toy food between characters in a picnic story [Eason], or pretended to be 'detectives' discovering the 'secrets' of shapes [Fisher 2011b, 2013]. In some studies, while the term guided play was not ascribed to the interventions, children engaged in play-based activities with adult guidance/scaffolding [Aleid; Bennett; Hawes; Starkey]. For example, teachers explained to children how PMs are like toys/games, and the authors acknowledged that teachers play a role in facilitating and encouraging children's active participation in learning [Aleid]. In another study, children engaged in a playful math activity with storytelling (e.g., using mice PMs after reading a story about mice), which teachers verbally scaffolded [Bennett]. Finally, in some interventions children engaged in hands-on discovery-based learning and experimentation (filling containers with various materials to learn about volume and capacity; [Dennis; Sophian]).

PMs are commonplace in math classrooms, as they provide children with opportunities to learn about abstract (i.e., symbolic) math concepts with concrete/tangible materials (representations). This is known as the concrete-to-abstract or concrete-pictorial-abstract approach. It is influenced by the Piagetian theory that concrete-operational (literal) thinking is a prerequisite to formaloperational (abstract) thinking. Many papers in this category refer to the concrete-to-abstract approach to math learning and/or Piaget's theory of development in their theoretical framework/literature review [Aleid; Alghazo; Alshehri; Bennett; Cramer; Dennis; Ermakova; Fisher 2011a, 2011b, 2013; Gecu-Parmaksiz; Horan; Hull; Martin; Mattoon; Moyer-Packenham; Sophian; Starkey; Taylor; Thompson 2012]. For example, children may first learn about fractions using PMs by placing five $1 / 5^{\text {th }}$ fraction tiles side-by-side to see that they are equal to a single larger fraction tile representing one whole. However, PMs may not support learning by themselves, and the accompanying activity and/or guidance on how to use them may be critical for children gaining a conceptual understanding (i.e., making connections between concrete objects and the abstract math concepts that they represent, e.g., [Dennis; Martin]).

\section{Evidence relating to effectiveness}

Most studies assessed performance on math/numeracy outcomes (e.g., fractions, volume/capacity, symbolic and non-symbolic magnitude comparison, counting, probability, and general math attainment/ability). Other outcome measures were related to math: three studies included language measures assessing math, spatial, and shape talk, and three administered visual-spatial tasks (mental rotation, spatial perception, and visual-spatial geometry). For an overview of main outcome domains and findings organised by study, see Appendix D (section 7.4.2). 
Seven papers compared physical and virtual (computerised) manipulatives, typically focusing on specific math concepts and materials (e.g., learning about fractions with fraction circles). Though the modality of the materials differed across conditions, the learning content and types of materials remained constant. For example, children classified concrete or digital geometric shapes [GecuParmaksiz], solved fraction problems with physical or virtual fraction circles/pies and tiles [MoyerPackenham], or learned about probability with real or computer-simulated coins and dice [Taylor]. These comparisons may provide mechanistic insight into whether the physical, hands-on nature of PMs is important in math interventions. Most found that PMs were not better than virtual manipulatives for improving math learning (e.g., fractions, probability) [Alshehri; Gecu-Parmaksiz; Mattoon; Mendiburo; Moyer-Packenham; Taylor] or visual-spatial skills [Gecu-Parmaksiz]. In fact, two studies found that children who engaged with virtual manipulatives outperformed those who used PMs [Gecu-Parmaksiz; Mendiburo]. Another study found that children who engaged with both standard and alternative physical shapes produced more spatial talk than children who engaged with just standard physical shapes or virtual shapes only [Verdine].

Greater gains for virtual than physical materials could be mediated by the automatised nature of computerised activities. While teachers must monitor and guide multiple pupils in a classroom environment on how to use PMs and provide feedback, app-based activities can provide instructions and instant feedback to individual pupils. The self-paced nature of computerised activities also enables children to progress at their own speed; in one study children who used virtual versus physical materials had more practice with math problems as they were able to complete more activities [Mendiburo].

The most promising findings were identified in reports investigating curriculum-based math interventions involving PMs. A large-scale effectiveness trial in Belize compared the impact of a math curriculum with PMs to regular math instruction: teachers in the intervention group received training on how to incorporate PMs and inquiry-based hands-on activities into math lessons, whereas control teachers received training about character development and positive discipline [Hull]. Math instruction in developing countries such as Belize is typically teacher-directed, with math concepts being taught using abstract (symbolic) representations, which can be difficult for children to learn and apply to novel problems [Hull]. Instead, the authors implemented a relatively simple and lowcost intervention 20 that departed from traditional approaches - one that was child-centred and involved PMs coupled with guidance from a teacher. Of note, is the robust methodology of this study: the authors employed an RCT design, measured outcomes at pre- and post-test, and included a large sample $(N \sim 6,600)$ of primary-age pupils spanning eight school grades. Furthermore, hierarchical linear (multi-level) modelling accounted for clustering at the pupil-, teacher-, and school-level, and controlled for baseline scores. Results from an intent-to-treat model found that children in the intervention group outperformed controls on eight age-appropriate math achievement tests. Positive effects were also associated with higher levels of teacher implementation (i.e., intervention fidelity) ${ }^{21}$. The benefits may have been mediated by the novelty of the child-centred curriculum being implemented in a developing country: there was a marked contrast between the control group

\footnotetext{
${ }^{20}$ Using PMs that could be fabricated by teachers.

${ }^{21}$ The positive effects also prompted a nationwide rollout of the intervention in Belize by the Ministry of Education.
} 
(traditional teaching, which is typically didactic) and the intervention group (a hands-on approach that encouraged children's active involvement in learning using PMs) [Hull]. The same approach may not be as effective relative to regular teaching practices in a more developed country such as the USA, in which child-centred approaches are largely accepted.

In another study, pre-school children participated in a math curriculum involving a wide range of hands-on activities and materials (e.g., sorting objects by size, filling containers, filling shapes with other shapes; [Sophian]). Intervention children outperformed passive and active (an alternative literacy curriculum) controls on math measures after controlling for baseline scores. Similarly, children who participated in a pre-kindergarten classroom- and home-based math curriculum involving playful activities with PMs (e.g., equally sharing toy bananas between two monkeys, making shapes, arranging animal cards by set size) outperformed passive controls on a measure of math skills [Starkey] ${ }^{22}$. While the benefits of math curriculums involving PMs on children's math ability are promising, it is unclear whether the hands-on activities are crucial for positive effects. Improvements in these studies could simply reflect exposure to math content in the interventions, as they are based on comparisons to passive controls or an alternative non-math (literacy) curriculum.

On the other hand, some curriculum-based studies compared learning of the same (or similar) math content with or without PMs. They demonstrated that learning with hands-on materials benefits volume and capacity learning [Dennis], fraction learning and retention [Cramer] ${ }^{23}$, and counting skills [Alghazo] ${ }^{24}$. Three additional single- and two-session studies (non-curriculum-based) investigated the impact of solving the same math problems (related to proportional reasoning, fractions, or equivalence) with or without PMs (magnetic strips, fraction tiles, blocks versus paperand-pencil activities/worksheets), but none found differences between these groups [Fujimura; Martin; Watchorn].

Other studies investigating the impact of curriculum-based math interventions with PMs have produced null or inconclusive results. There were no group differences in the math ability scores of a group who engaged in teacher-scaffolded math tasks, involving storytelling and PMs, and controls who received traditional teaching [Bennett]. In another study, while children in two groups who received math instruction with $\mathrm{PMs}$ or multimedia/videos improved more than controls (traditional teaching) on a math measure of geometry and visualisation skills, there were no other group differences [Thompson 2012]. Finally, a curriculum-based spatial geometry intervention resulted in selective benefits to a visual-spatial geometry measure when compared to an inquiry-based control approach involving scientific topics [Hawes]. However, while time by group interactions (ANOVA) indicated greater gains for the intervention group on another spatial measure (mental rotation), spatial language, and symbolic (number) magnitude comparison, post hoc pairwise comparisons were either not conducted or found no effects for these outcomes. There were also no group

\footnotetext{
${ }^{22}$ However, no baseline data was collected for the comparison group. [Starkey] also reported that middle-income children receiving the intervention outperformed low-income children at pre- and post-test, but the lower-income children showed greater gains both groups showed gains, and an interaction revealed that the low-income sample had greater gains and caught-up to middle-income children who did not receive the intervention.

${ }^{23}$ However, no pre-test data was collected in this study.

${ }_{24}$ The intervention in this study involved PMs and computerised media (e.g., cartoon animations), and so it is unclear whether one or both approaches were critical for the observed benefits to counting ability.
} 
differences for other math (non-symbolic [dot] magnitude comparison and number knowledge) or language (receptive vocabulary) measures.

Several studies compared interventions with varying levels of adult guidance, child autonomy, and physical engagement, and produced mixed results. Two studies compared guided play with concrete shapes to free play or didactic learning (in which children could see but not touch the shapes). Guided play was found to benefit shape sorting performance more than the other two groups [Fisher, 2011b; Fisher, 2013], and improved performance on an embedded shapes task relative to free play [Fisher, 2011b]. In another study by the same authors, guided play was compared to both didactic learning and reading control conditions. However, there were no group differences on an embedded shapes task, and both guided play and didactic learning benefited shape sorting performance more than reading. In a similar study, children learned about whole and parts during guided play: after parentchild dyads read a story about a picnic, they shared segments of toy food among characters in the story [Eason]. This condition was compared to free play with the same materials or formal learning (no touching). Children in the formal learning group produced more math talk than the other two groups, and those in the guided play condition engaged in more math talk than free play controls. Overall, findings from guided play studies were mixed and inconclusive, and potentially limited by methodological shortcomings: none employed a pre-post design (i.e., they collected data at post-test or during the intervention activity only) and all involved just a single intervention session ${ }^{25}$.

An additional study compared four conditions that all involved coin-based PMs with varying degrees of adult guidance but did not identify any group differences in counting scores [Horan]. Finally, PMs (fraction bars, base-10 blocks) plus peer tutoring benefited math attainment more than PMs alone or regular teaching [Aleid], and there were no differences in the likelihood of children employing a base10 addition strategy following an intervention with single versus multiple PM representations (e.g., base-10 frames or base-10 frames plus tiles) [Ermakova].

\section{Summary}

\section{Key findings}

Interventions involving math-based PMs comprised the largest grouping of studies in the review, likely owing to the well-established use of concrete materials during traditional math instruction. Most studies acknowledged the concrete-to-abstract (or concrete-pictorial-abstract) approach for enhancing children's understanding of math concepts - in which children are first introduced to an abstract math concept, such as addition or subtraction, using concrete resources (PMs), then pictorial representations, and finally abstract symbols.

There is evidence that math interventions involving PMs benefit children's learning, as measured by math or math-related outcomes, with positive effects reported for both didactic and play-based approaches. Promising findings were reported for curriculum-based programmes involving math PMs: intervention children outperformed controls on measures of math achievement [e.g., Dennis; Hull; Sophian; Starkey], with additional evidence in one study that gains were mediated by intervention fidelity [Hull]. Of note, is the robust methodology of the large-scale trial that took place

\footnotetext{
${ }^{25}$ Note that two of the curriculum-based studies were not explicitly conceptualised as guided play interventions but could nonetheless be classified as such [Hawes; Bennett].
} 
in schools in Belize - a lower-middle-income country [Hull]. However, numerous studies have also reported null or inconclusive results.

Overall, findings relating to effectiveness were mixed, in part due to methodological differences across studies. These differences also limit the conclusions that can be drawn about the intervention components that are critical for mediating any observed learning gains (e.g., whether the physical nature of activities is crucial). Most studies that compared physical and computerised activities found that PMs were no better, or worse, than virtual materials, meaning that the physical nature of the task may not be critical. Notwithstanding, PMs are useful tools for introducing and practicing math concepts; they can be used to encourage children's active engagement in math learning through hands-on activities and to foster a more playful learning environment.

Key gaps \& recommendations for future research

- Studies were overwhelmingly conducted in the USA. There is a pressing need for more geographically diverse research, especially in low- and middle-income countries.

- Overall, studies were limited by methodological inconsistencies and shortcomings. There is a need for studies with more robust research designs that can tease out the selective benefits of PMs (e.g., to understand whether virtual materials are always better than physical materials for math learning). 
Table 2. Characteristics of studies in the math PMs category $(n=26)$.

\begin{tabular}{|c|c|c|c|c|c|c|c|c|c|c|c|c|}
\hline ID & Location & $\underset{N}{\text { Sample }}$ & Age & $\begin{array}{l}\text { Research } \\
\text { design }\end{array}$ & Setting & $\begin{array}{l}\text { Model of } \\
\text { delivery }\end{array}$ & $\begin{array}{c}\text { Adult } \\
\text { involved }\end{array}$ & $\begin{array}{c}N \\
\text { sessions }\end{array}$ & Materials & Intervention & Comparison(s) & $\begin{array}{c}\text { Main } \\
\text { outcome } \\
\text { domains }\end{array}$ \\
\hline $\begin{array}{l}\text { Aleid } \\
2015\end{array}$ & $\begin{array}{l}\text { Saudi } \\
\text { Arabia }\end{array}$ & 622 a & $\begin{array}{l}10- \\
12\end{array}$ & RCT, pre-post & $\begin{array}{l}\text { School/ } \\
\text { classroom }\end{array}$ & $\begin{array}{l}\text { Class/ } \\
\text { pairs }\end{array}$ & Teacher & $11-20$ & $\begin{array}{l}\text { Fraction bars, } \\
\text { base-10 blocks }\end{array}$ & $\begin{array}{l}\text { Teachers explained the value of PMs \& } \\
\text { how they are like toys. Children } \\
\text { physically explored \& used them in } \\
\text { fraction/decimal tasks. Pairs of children } \\
\text { alternated a peer tutor role }\end{array}$ & $\begin{array}{l}\text { 1. PMs alone } \\
\text { 2. Peer tutoring alone } \\
\text { 3. No-intervention }\end{array}$ & $\begin{array}{c}\text { Math/ } \\
\text { numeracy }\end{array}$ \\
\hline $\begin{array}{c}\text { Alghazo } \\
2010\end{array}$ & $\begin{array}{l}\text { United } \\
\text { Arab } \\
\text { Emirates }\end{array}$ & 48 & $4-6$ & QE, pre-post & $\begin{array}{l}\text { School/ } \\
\text { classroom }\end{array}$ & Class & Teacher & $>20$ & $\begin{array}{l}\text { Blocks \& other } \\
\text { objects }\end{array}$ & $\begin{array}{l}\text { Counting tasks with concrete (e.g., } \\
\text { ordering, counting), pictorial, \& } \\
\text { computer-based (text, audio, visuals) } \\
\text { materials. Unclear how much involved } \\
\text { PMs vs. computer-based }\end{array}$ & No-intervention & $\begin{array}{c}\text { Math/ } \\
\text { numeracy }\end{array}$ \\
\hline $\begin{array}{l}\text { Alshehri } \\
2018\end{array}$ & $\begin{array}{c}\text { Saudi } \\
\text { Arabia }\end{array}$ & $163 \mathrm{~b}$ & $\begin{array}{c}10- \\
12\end{array}$ & QE, pre-post & $\begin{array}{l}\text { School/ } \\
\text { classroom }\end{array}$ & $\begin{array}{l}\text { Class/ } \\
\text { group }\end{array}$ & Teacher & $2-5$ & Fraction bars & $\begin{array}{l}\text { Children used PMs to help solve math } \\
\text { fraction problems }\end{array}$ & $\begin{array}{l}\text { 1. Virtual } \\
\text { manipulatives } \\
\text { 2. No-intervention }\end{array}$ & $\begin{array}{c}\text { Math/ } \\
\text { numeracy }\end{array}$ \\
\hline $\begin{array}{l}\text { Bennett } \\
2000\end{array}$ & USA & 57 & $4-6$ & QE, pre-post & $\begin{array}{l}\text { Childcare } \\
\text { centre }\end{array}$ & Group & Teacher & $>20$ & $\begin{array}{l}\text { Shapes, } \\
\text { counters, buttons, } \\
\text { dominoes, etc }\end{array}$ & $\begin{array}{l}\text { Storytime \& PM-based activities (e.g., } \\
\text { counting, sorting, length estimation) that } \\
\text { teachers verbally scaffolded }\end{array}$ & Traditional teaching & $\begin{array}{c}\text { Math/ } \\
\text { numeracy }\end{array}$ \\
\hline $\begin{array}{l}\text { Cramer } \\
2002\end{array}$ & USA & 1,666 & $\begin{array}{c}10- \\
12\end{array}$ & RCT, post-only & $\begin{array}{l}\text { School/ } \\
\text { classroom }\end{array}$ & $\begin{array}{l}\text { Class/ } \\
\text { group }\end{array}$ & Teacher & $>20$ & $\begin{array}{l}\text { Fraction circles, } \\
\text { chips, paper for } \\
\text { folding }\end{array}$ & $\begin{array}{l}\text { The Rational Number Project (RNP): a } \\
\text { curriculum-based intervention involving } \\
\text { PMs for learning fractions }\end{array}$ & $\begin{array}{l}\text { Traditional teaching } \\
\text { (textbook \& PMs to } \\
\text { lesser extent) }\end{array}$ & $\begin{array}{c}\text { Math/ } \\
\text { numeracy }\end{array}$ \\
\hline $\begin{array}{l}\text { Dennis } \\
2012\end{array}$ & USA & 20 & $\begin{array}{c}10- \\
12\end{array}$ & QE, pre-post & $\begin{array}{l}\text { School/ } \\
\text { classroom }\end{array}$ & Class & Teacher & $>20$ & $\begin{array}{l}\text { Containers (e.g., } \\
\text { bottles, cups) \& } \\
\text { various materials } \\
\text { (e.g., rice, water) }\end{array}$ & $\begin{array}{l}\text { Hands-on activities with PMs (e.g., filling } \\
\text { containers with materials) to learn } \\
\text { concepts like volume, area, length, etc }\end{array}$ & $\begin{array}{l}\text { Same as intervention } \\
\text { without PMs }\end{array}$ & $\begin{array}{c}\text { Math/ } \\
\text { numeracy }\end{array}$ \\
\hline $\begin{array}{l}\text { Eason } \\
2020\end{array}$ & USA & 72 & $4-6$ & $\begin{array}{l}\text { RCT, during- } \\
\text { only }\end{array}$ & $\mathrm{Lab}$ & Individual & $\begin{array}{l}\text { Parent/ } \\
\text { caregiver }\end{array}$ & 1 & $\begin{array}{l}\text { Segmented toy } \\
\text { food }\end{array}$ & $\begin{array}{l}\text { Parent-child dyads played with } \\
\text { segmented toy food to explore concepts } \\
\text { like whole \& parts. They read a story } \\
\text { about a picnic \& divided food between } \\
\text { characters to encourage physical } \\
\text { engagement with PMs }\end{array}$ & $\begin{array}{l}\text { 1. Free play with } \\
\text { same materials } \\
\text { 2. Formal learning } \\
\text { (could see but not } \\
\text { touch PMs) }\end{array}$ & $\begin{array}{l}\text { Literacy/ } \\
\text { language }\end{array}$ \\
\hline $\begin{array}{l}\text { Ermakova } \\
\quad 2017\end{array}$ & USA & 61 & $4-6$ & RCT, pre-post & $\begin{array}{l}\text { School/ } \\
\text { classroom }\end{array}$ & Group & Researcher & $2-5$ & $\begin{array}{l}\text { Base-10 frames \& } \\
\text { tiles }\end{array}$ & $\begin{array}{c}\text { Children shown how to use the math } \\
\text { PMs by a researcher during training } \\
\text { sessions }\end{array}$ & $\begin{array}{l}\text { Same as intervention } \\
\text { with only frames or } \\
\text { tiles }\end{array}$ & $\begin{array}{c}\text { Math/ } \\
\text { numeracy }\end{array}$ \\
\hline $\begin{array}{l}\text { Fisher } \\
2011 \mathrm{a}^{\mathrm{c}}\end{array}$ & USA & 42 & $4-6$ & RCT, post-only & Lab & Individual & Researcher & 1 & Geometric shapes & $\begin{array}{l}\text { Children shown shape cards and } \\
\text { encouraged to 'discover' their 'secrets' } \\
\text { (properties) by touching/tracing them }\end{array}$ & $\begin{array}{l}\text { 1. Didactic instruction } \\
\text { (adult showed shape } \\
\text { features, no touch) } \\
\text { 2. Reading }\end{array}$ & $\begin{array}{c}\text { Math/ } \\
\text { numeracy }\end{array}$ \\
\hline $\begin{array}{l}\text { Fisher } \\
2011 b \mathrm{~d}\end{array}$ & USA & 38 & $4-6$ & RCT, post-only & $\begin{array}{l}\text { School/ } \\
\text { classroom }\end{array}$ & Individual & Researcher & 1 & $\begin{array}{l}\text { Geometric shapes } \\
\text { \& construction } \\
\text { sticks }\end{array}$ & $\begin{array}{l}\text { As above, but with an additional activity } \\
\text { (creating new shapes with sticks) }\end{array}$ & $\begin{array}{l}\text { 1. Didactic instruction } \\
\text { (adult identified } \\
\text { shape features, no } \\
\text { touch) }\end{array}$ & $\begin{array}{c}\text { Math/ } \\
\text { numeracy }\end{array}$ \\
\hline
\end{tabular}




\begin{tabular}{|c|c|c|c|c|c|c|c|c|}
\hline $\begin{array}{l}\text { Fisher } \\
2013^{\text {e }}\end{array}$ & USA & 60 & $4-6$ & $\begin{array}{l}\text { Unclear, post- } \\
\text { only }\end{array}$ & $\begin{array}{l}\text { School/ } \\
\text { classroom } \\
\text { \& lab }\end{array}$ & Individual & Researcher & 1 \\
\hline $\begin{array}{c}\text { Fujimura } \\
2001\end{array}$ & Japan & 76 & $7-9$ & RCT, pre-post & $\begin{array}{c}\text { School/ } \\
\text { classroom }\end{array}$ & Individual & Researcher & 1 \\
\hline $\begin{array}{c}\text { Gecu- } \\
\text { Parmaksiz } \\
2018, \\
2019 \mathrm{f}\end{array}$ & Turkey & 72 & $4-6$ & RCT, pre-post & $\begin{array}{l}\text { School/ } \\
\text { classroom }\end{array}$ & Group & Teacher & $2-5$ \\
\hline $\begin{array}{c}\text { Hawes } \\
2017\end{array}$ & USA & 65 & $4-6$ & QE, pre-post & $\begin{array}{c}\text { School/ } \\
\text { classroom }\end{array}$ & Class & Teacher & $>20$ \\
\hline
\end{tabular}

Horan

2018

USA

165 4-6 RCT, pre-post School/ classroom

Group Researcher

$2-5$

Coins and paper- Counting numbers 1-10 with PMs \& printed coin strips

placing them on a board (with level of adult-guidance varying by group)

Geoboards, Curriculum-based programme, teachers geometric solids, trained on using PMs \& inquiry-based flash cards, hands-on math activities (some involved counters collaboration in small groups)

$$
\text { collaboration in small groups) }
$$

Tasks involving PMs to solve fraction Same as intervention problems (e.g., to represent $1 / 3$ of 6 ), the but using pictures adult provided feedback $\&$ hints instead of PMs

Virtual manipulatives

Cubes, dice, toy counters

Children complete math tasks with PMs
\& the teacher provided feedback

math problems via tablet computer, automatic feedback)

$\begin{array}{ccc}\text { Children engaged in fraction } & \text { Virtual fraction } \\ \text { Paper fraction } & \text { games/activities with fraction PMs that } & \text { activities with digital }\end{array}$ strips they constructed using paper manipulatives Fraction pies \& $\begin{gathered}\text { Teachers demonstrated how to use PMs } \\ \text { \& children used them to complete }\end{gathered}$ wildren used them to complete (via tablet computer) numeracy
worksheet fraction problems tiles

Counters, tiles, Curriculum-based intervention: new 1. Literacy curriculum Math/ scales, containers, theme each week (e.g., volume/capacity, 2. No-intervention numeracy

Math/ numeracy

\section{Math/}

numeracy

Visualspatial, math/ numeracy

Math/

numeracy, math-spatial receptive \&

spatial language

Math/ numeracy

Math/ numeracy

Math/ numeracy

Math/ numeracy Math/ numeracy

Moyer2013
$>20$ problems, no PMs)

learning about

science (e.g., gravity,

weather, lifecycles)

degrees of adult

Traditional math

2004 $\begin{array}{cccc}\text { QE, } & \begin{array}{c}\text { School/ } \\ \text { classroom }\end{array} & \begin{array}{c}\text { Class/ } \\ \text { group/ }\end{array} & \begin{array}{c}\text { Teacher \& } \\ \text { parent/ }\end{array}\end{array}$ 
\& home individual caregiver

\section{Taylor}

2001
QE, pre-post

\& post-only

(control)

School/ Class/ Teacher \& classroom group/ parent/
USA

$66 \begin{array}{cc}10- & \text { QE, pre-post } \\ 12\end{array}$

School/ Class/

classroom group

$\begin{gathered}\begin{array}{c}\text { Thompson } \\ 2012\end{array} \\ \text { USA }\end{gathered} \quad 157 \quad 4-6 \quad$ QE, pre-post $\begin{gathered}\text { School/ } \\ \text { classroom }\end{gathered}$ Class $\quad$ Teacher $\quad>20 \quad \begin{gathered}\text { 3D geometric } \\ \text { shapes }\end{gathered}$

Verdine 2019

USA
$60 \quad 0-3 \quad$ RCT, during- only

Lab Individual Parent/

caregiver

Watchorn
2011

$7-9$

2011

Canada

board/card games, shapes/geometry, weight) \& related PM puzzles, geometric activities (e.g., sorting by size, filling

shapes, \& others containers, using tiles to calculate area)

Pre-K math curriculum with PMs:

various topics (e.g., number, pattern, spatial, arithmetic, measurement),

Shapes, counters, teacher-guided group activities (e.g. others

lengths, arranging cards by set size) computer-based activities \& parentguided tasks at home

Activities with PMs about probability \& Coins, dice, cubes, chance (e.g., tossing coins, rolling dice, \& spinners, marbles making predictions)

Activities with 3D shapes (e.g., combining them to make complex objects, working out which could stack/slide/roll or how many faces/edges they had, comparing plane/solid figures)

Parent-child dyads played with standard

(e.g., triangle, circle) \& alternate shapes 1 Geometric shapes (e.g., long/thin triangle). Parents were (\& told about some suggested activities)

Adult-directed instruction with PMs:

2-5 Cylindric wooden children placed blocks in trays to match a math (equivalence) problem as

\section{blocks \& trays}

$$
\text { directed }
$$
sticks, cards, \& making 2D \& 3D shapes, comparing

No-intervention

(successive cohort design)

Math/ numeracy

1. Computerised

manipulatives (e.g.,

dice, coins)

2. Physical \&

computerised

manipulatives

3. Traditional

teaching

\section{Math/}

numeracy

timedia (videos

about shapes)

2. PMs \& multimedia Math-spatial

3. No-intervention

1. PMs (standard shapes)

2. Virtual task

Spatial

(standard shapes,

tablet computer)

1. No PMs (same math

problems)

2. Instruction on unrelated math topic

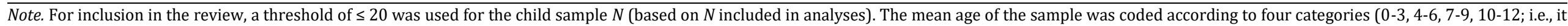

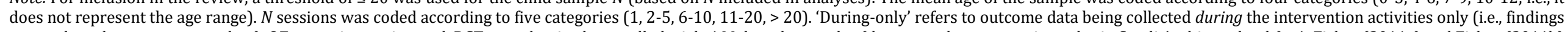

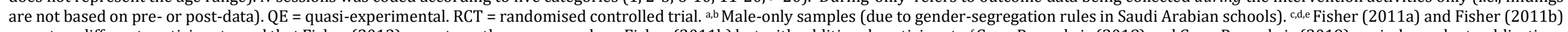

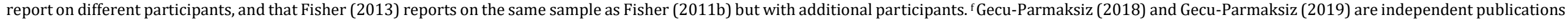

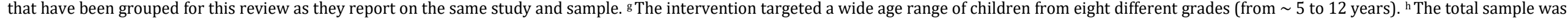

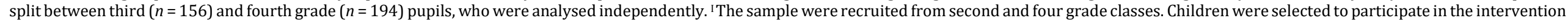
based on their pre-test scores (children who failed equivalence problems in the first study session). 


\subsubsection{Reading/literacy-based PMs ( $n=8)$}

\section{KEY FINDINGS}

- PMs typically comprised small toys \& figurines linked to target vocabulary or phonics.

- There was some evidence of improvements to children's vocabulary, literacy skills, \& memory for story content, but overall, effects were mixed \& null results were also reported.

- Most studies involved guided play; positive effects were reported following both guided \& direct instruction with PMs.

\section{Overview}

This section summarises literacy/reading interventions involving PMs, which were all conducted in the USA. See Table 3 for a summary of study characteristics. The level of physical engagement across studies ranged from moderate to low. Children did not use PMs for construction or otherwise creating something, rather they typically engaged with small toys and figurines related to a story. In some they had a high degree of choice in what they could do with the materials (e.g., enacting a picnic scenario; [Dickinson]), though the nature of the props likely limited the amount of physical manipulation that was possible. In others, the actions children performed with PMs were more prescribed [e.g., Biazak]. Like in the previous sections, the degree of freedom children had to physically interact with PMs was associated with the level of adult guidance (i.e., more adult direction = less opportunity for children to physically explore or manipulate the materials).

\begin{tabular}{|ll|}
\hline \multicolumn{2}{|c|}{ KEY INTERVENTION FEATURES } \\
Location & USA (8) \\
Setting & School/classroom (7), childcare centre (1) \\
Adult involved & Researcher (6), teacher (2) \\
Main outcome domains & Literacy/language (7), memory (1), social- \\
& emotional (1)
\end{tabular}

\section{Interventions}

In most interventions, children engaged in a joint reading activity with an adult (researcher or teacher) and played with figurines and small toy props related to story content [Biazak; Dickinson; Han; Toub; Weisberg]. For example, children might re-enact what happened in a story by 'baking a cake' with the materials [Biaszak; Dickinson; Han]. During play sessions with PMs, the adult involved would facilitate language learning or comprehension by incorporating and focusing on target vocabulary, elaborating on children's talk, asking closed- and open-ended questions, and/or using definitions [Dickinson; Toub; Weisberg]. The remaining three studies involved different types of PMs and activities: small toys/objects were used as memory aids to prompt comprehension strategies 
during reading [Cobb] or as phonics-related items to use in child-invented literacy games [Cavanaugh], and PM letters were used in phoneme segmenting tasks [Lane].

Five studies were conceptualised as guided play interventions, with varying degrees of adult guidance and child autonomy. The implementation of guided play was most evident in two studies. In one, children's play with story-relevant PMs was scaffolded by an adult who followed their lead, engaged in co-play, asked questions, and incorporated, defined, and modelled the use of target words at naturally occurring moments [Toub]. In the other, children could express their creativity and collaborate with peers by inventing their own PM-based phonics games [Cavanaugh]. The others also involved adult-guided play-based learning, but afforded children relatively less freedom within their play. For example, an adult modelled actions for target words from the story and children would use toy props to act out the target words during play sessions [Dickinson; Han; Weisberg]. Other interventions involved more prescribed, adult-directed activities, with limited child choice [Biazak; Cobb; Lane].

Across the studies, there were differences in the theoretical rationale for including PMs in literacy/reading interventions. One provided a detailed overview supporting the potential benefits of physical interaction with PMs for children's language comprehension, drawing on developmental and cognitive theories such as Bruner's enactive-iconic-symbolic learning modes, Piaget's theory of concrete-to-abstract thinking, and embodied and indexical learning [Biazak]. Based on these accounts, young children learn through physical interaction with their environment, which facilitates their construction of meaning (i.e., comprehension). Young children may struggle to understand symbolic representations (words), but providing them with concrete materials and enactive representations (though hands-on activities with PMs in the presence of words), may improve their comprehension [Biazak]. Another study also alluded to using objects as meaningful concrete referents of target vocabulary [Han].

Two studies used PMs for more specific reasons - to help children learn about 'word decoding' (i.e., building words with PM letters to see connections between letters, sounds, syllables, and chunks in new words and in words they already know; [Lane]), or as prompts for reading comprehension strategies [Cobb]. In the remaining four studies, PMs were somewhat incidental materials in playbased activities [Cavanaugh; Dickinson; Toub; Weisberg]. In these interventions, PMs were related to story content, target vocabulary, or phonemes, and when integrated with play provided a meaningful context for children to socially interact and to practice their literacy/language skills.

\section{Evidence relating to effectiveness}

All the studies assessed children's literacy/language at outcome (using measures of early literacy skills, reading, and receptive and expressive vocabulary), except one that measured children's memory retention for story content [Biazak]. One study also tested children's self-regulation skills [Dickinson]. The research aims varied across studies, meaning that different comparison groups were used: some manipulated the level of instruction (e.g., guided play versus direct instruction; [Cavanaugh; Han]) and/or the inclusion of PMs (e.g., reading with versus without PMs; [Biazak]), while others manipulated neither of these task features [e.g., Weisberg]. This limits the conclusions that can be drawn regarding the relative importance of PMs and/or the level of adult guidance/child autonomy. Although all the studies employed an experimental pre- and post-test design, their 
analytic methods differed: some used more rigorous techniques (i.e., that controlled for pre-test scores and/or nesting in classrooms; [Dickinson; Biazak; Lane; Toub]), while others used less robust methods (i.e., $t$-tests that do not account for the potential impact of baseline differences between groups, e.g., [Cobb; Han]). Further details regarding the findings of each study can be found in Appendix D (section 7.4.3).

There was some evidence that literacy-rich guided play interventions benefited children's literacy and vocabulary learning, however findings were mixed and inconclusive. Children showed greater improvements in early literacy skills immediately after receiving a guided play versus adult-directed intervention (both with PMs) [Cavanaugh]. In another study, both guided- and directed-play with PMs resulted in benefits to receptive and expressive vocabulary scores relative to a free play condition with PMs [Toub]. However, in contrast with the findings of [Cavanaugh], there were no differences between the guided and directed play groups [Toub]. Finally, benefits were reported to children's receptive but not expressive vocabulary following guided play versus direct instruction with PMs [Han]. In the three studies, all comparison conditions involved PMs and so it remains unclear whether they are critical for any of the reported intervention effects.

The research design of [Cavanaugh] also provided some unique insights from teachers of children who completed guided play with PMs and direct instruction with PMs consecutively (two groups engaged in guided play for the first half of the study, and then direct instruction for the second half, or vice versa). One teacher reported that children who switched from the play-based to prescribed activity were disappointed, and those who moved to the play-based group enjoyed the increased opportunities for exploration and making their own games. The teacher reflected on giving children more control over their learning: while they were initially hesitant, they were ultimately surprised at how little adult interaction they needed. Another teacher observed children's positive attitudes, eagerness, and enjoyment during the play-based learning activities.

There was limited evidence for the benefit of reading interventions involving PMs when compared to reading interventions without PMs. Children who moved toys to enact what characters in a story were doing remembered more story content than those who simply listened [Biazak]. However, a another reading intervention with PMs was no better than a reading programme without PMs for enhancing children's receptive or expressive vocabulary, or their self-regulatory skills [Dickinson].

In one study, two groups of children engaged in a guided play reading intervention that involved enactment of either fantasy or realistic story content with PMs [Weisberg]. While both groups demonstrated gains in expressive and receptive vocabulary (with no differences found between the fantasy and realistic groups), there were no other comparison conditions meaning that observed benefits cannot be attributed to guided play or PMs (e.g., they could simply reflect practice effects).

In the remaining two studies, literacy interventions involving PMs were compared to passive control groups (business-as-usual), meaning that findings must be interpreted with caution. While positive findings are promising - suggesting that a hands-on intervention with PMs might be better, or at least just as good as, regular teaching methods - they could be explained by other extraneous factors (e.g., expectancy effects). A study that examined the impact of a literacy tutoring programme with or 
without certain features relative to a no-intervention condition ${ }^{26}$ found that all four intervention groups (both with and without PMs) outperformed passive controls on a measure of phonological awareness [Lane]. Furthermore, children who received the whole intervention (but not the other intervention groups) scored higher than controls on a sight word task. However, critically, no pairwise comparisons between the intervention groups themselves were reported (e.g., the tutoring programme with PMs versus the same programme without PMs). In another study, first graders' reading scores were better than those of controls following a comprehension strategy intervention involving PMs [Cobb]. However, this benefit was not found for second or third grade children.

\section{Summary}

\section{Key findings}

There is a growing body of literature examining the impact of reading interventions involving PMs on children's literacy skills, though PMs are not as commonly implemented in this domain as they are for math learning (see section 2.5.2). There was some evidence for positive effects on children's vocabulary, literacy skills, and memory for story content, however findings were inconsistent, with studies also reporting null effects. Furthermore, methodological variations across studies make it difficult to draw conclusions regarding the relative importance of certain intervention features such as play-based instruction or PMs. Interestingly, there was some overlap between the theoretical rational for the use of PMs in literacy and math instruction (see section 2.5.2), whereby it is proposed that PMs can be used as concrete referents of abstract concepts/symbols (e.g., words and letters). Most studies involved guided play, suggesting that PMs may lend themselves to playful approaches for literacy education. During literacy-rich, hands-on play with PMs, children experience opportunities for active participation, social interaction, collaboration, and naturally occurring moments for language learning.

A limitation of this review in general is that there was no systematic extraction of qualitative data due to limited resources, meaning that potential information pertaining to participants' perceptions of PMs and play-based practices have not been captured. Nevertheless, a promising finding suggests that teachers who might initially be hesitant in relinquishing control to children in the classroom, ultimately recognise the value of play-based methods and acknowledge children's enjoyment and excitement in these learning activities with PMs.

Key gaps \& recommendations for future research

- There was a notable absence of literature outside of the USA, highlighting the pressing need to diversify the geographical scope of literacy interventions involving PMs, especially in lower-income contexts. Future research could examine if PMs are effective in promoting more playful literacy/reading teaching practices in these contexts.

- Further research is could also explore the additive value of PMs in reading interventions independent of other programme features.

\footnotetext{
${ }^{26}$ This study examined a one-on-one literacy tutoring intervention with different components, including book reading plus: (1) PM letter activities, (2) writing, and (3) reading additional sources (e.g., newspapers) [Lane]. Children either received the whole programme (with all three elements), or a version of the intervention without one of the components, yielding four intervention groups (one of which did not involve PMs), which were each compared to no-intervention condition but not to each other.
} 
PEDAL Report: PM scoping review

Table 3. Characteristics of studies involving reading with PMs $(n=8)$.

\begin{tabular}{|c|c|c|c|c|c|c|c|c|c|c|c|c|}
\hline ID & Location & $\begin{array}{c}\text { Sample } \\
\quad N\end{array}$ & Age & $\begin{array}{l}\text { Research } \\
\text { design }\end{array}$ & Setting & $\begin{array}{l}\text { Model of } \\
\text { delivery }\end{array}$ & $\begin{array}{c}\text { Adult } \\
\text { involved }\end{array}$ & $\begin{array}{c}N \\
\text { sessions }\end{array}$ & Materials & Intervention & Comparison(s) & $\begin{array}{c}\text { Main } \\
\text { outcome } \\
\text { domains }\end{array}$ \\
\hline $\begin{array}{l}\text { Biazak } \\
2010\end{array}$ & USA & 56 & $4-6$ & RCT, pre-post & $\begin{array}{l}\text { Childcare } \\
\text { centre }\end{array}$ & Individual & Researcher & 1 & $\begin{array}{l}\text { Figurines/ } \\
\text { toy props }\end{array}$ & $\begin{array}{l}\text { Joint reading (story about a zoo) \& play } \\
\text { with related toys (e.g., people, chairs, } \\
\text { monkey, tree, bicycle, elephant). Children } \\
\text { moved toys to enact what characters in } \\
\text { the story were doing. }\end{array}$ & $\begin{array}{l}\text { Listening-only condition } \\
\text { (no PMs) }\end{array}$ & $\begin{array}{l}\text { Memory } \\
\text { (verbal) }\end{array}$ \\
\hline $\begin{array}{l}\text { Cavanaugh } \\
\quad 2017\end{array}$ & USA & 41 & $4-6$ & QE, pre-post & $\begin{array}{l}\text { School/ } \\
\text { classroom }\end{array}$ & Group & Teacher & $11-20$ & $\begin{array}{l}\text { Small } \\
\text { toys/items }\end{array}$ & $\begin{array}{l}\text { Following a teachers' example, children } \\
\text { were provided with phonics-related } \\
\text { items (e.g., a toy tiger for 't') \& } \\
\text { encouraged to create their own game } \\
\text { (e.g., sorting game, storytelling). }\end{array}$ & $\begin{array}{c}\text { Teacher-directed } \\
\text { instruction with the same } \\
\text { materials. }\end{array}$ & $\begin{array}{l}\text { Literacy/ } \\
\text { language }\end{array}$ \\
\hline $\begin{array}{l}\text { Cobb } \\
2001\end{array}$ & USA & 59 a & $\begin{array}{l}4-6 \\
7-9 b\end{array}$ & RCT, pre-post & $\begin{array}{l}\text { School/ } \\
\text { classroom }\end{array}$ & Individual & Researcher & $11-20^{c}$ & Toys/props & $\begin{array}{l}\text { Joint reading with PMs representing } \\
\text { 'strategies' such as 'read on' (toy car). The } \\
\text { adult could show an item to hint a } \\
\text { comprehension strategy (e.g., 'read on' to } \\
\text { see if the rest of the sentence provides a } \\
\text { clue to word meaning). Children had a } \\
\text { chance to play with the PMs. }\end{array}$ & $\begin{array}{l}\text { Business-as-usual (regular } \\
\text { literacy classes) }\end{array}$ & $\begin{array}{l}\text { Literacy/ } \\
\text { language }\end{array}$ \\
\hline $\begin{array}{l}\text { Dickinson } \\
2019\end{array}$ & USA & 227 & $4-6$ & RCT, pre-post & $\begin{array}{l}\text { School/ } \\
\text { classroom }\end{array}$ & Group & Teacher & $11-20 \mathrm{~d}$ & $\begin{array}{l}\text { Figurines/ } \\
\text { toy props }\end{array}$ & $\begin{array}{l}\text { Read-play-learn: after book reading, } \\
\text { teachers re-enacted the story with PMs. } \\
\text { After, children played with the PMs \& } \\
\text { teachers facilitated their language } \\
\text { focusing on target words (e.g., open- } \\
\text { ended questions) }\end{array}$ & $\begin{array}{l}\text { Reading without additional } \\
\text { play sessions }\end{array}$ & $\begin{array}{l}\text { Literacy/ } \\
\text { language, } \\
\text { social- } \\
\text { emotional }\end{array}$ \\
\hline $\begin{array}{l}\text { Han } \\
2010\end{array}$ & USA & 49 e & $4-6$ & RCT, pre-post & $\begin{array}{c}\text { School/ } \\
\text { classroom }\end{array}$ & Pairs & Researcher & $>20$ & Toy props & $\begin{array}{l}\text { EIVP \& play: joint book reading activity } \\
\text { in which the adult modelled actions } \\
\text { relating to target words using PMs, } \\
\text { followed by an adult-guided (modelling, } \\
\text { co-play, following child's lead) play } \\
\text { episode in which children acted out } \\
\text { target words with props (e.g., baking) }\end{array}$ & $\begin{array}{l}\text { Reading with PMs but no } \\
\text { additional play episodes }\end{array}$ & $\begin{array}{l}\text { Literacy/ } \\
\text { language }\end{array}$ \\
\hline $\begin{array}{l}\text { Lane } \\
2009\end{array}$ & USA & 100 & $4-6$ & RCT, pre-post & $\begin{array}{l}\text { School/ } \\
\text { classroom }\end{array}$ & Individual & Researcher & $>20$ & PM letters & $\begin{array}{l}\text { Early literacy intervention (full } \\
\text { programme) with adult-scaffolding: book } \\
\text { reading plus activity with PM letters, } \\
\text { writing, \& reading other sources (e.g., } \\
\text { newspapers) }\end{array}$ & $\begin{array}{l}\text { 1. No writing } \\
\text { 2. No PMs } \\
\text { 3. No other sources } \\
\text { 4. No-intervention }\end{array}$ & $\begin{array}{l}\text { Literacy/ } \\
\text { language }\end{array}$ \\
\hline $\begin{array}{l}\text { Toub } \\
2018\end{array}$ & USA & 249 & $4-6$ & RCT, pre-post & $\begin{array}{l}\text { School/ } \\
\text { classroom }\end{array}$ & Group & Researcher & $6-10$ & $\begin{array}{l}\text { Figurines/ } \\
\text { toy props }\end{array}$ & $\begin{array}{l}\text { After book reading children freely played } \\
\text { with PMs related to the story. The adult } \\
\text { guided the play episodes (followed the } \\
\text { child's lead, co-play, modelling) \& } \\
\text { incorporated target words at naturally }\end{array}$ & $\begin{array}{l}\text { 1. Reading \& free play (no } \\
\text { adult guidance) } \\
\text { 2. Reading \& adult- } \\
\text { directed play }\end{array}$ & $\begin{array}{l}\text { Literacy/ } \\
\text { language }\end{array}$ \\
\hline
\end{tabular}




\section{PEDAL Report: PM scoping review}

occurring moments (using definitions,

closed- \& open-ended questions)

Joint reading \& play with PMs related to a Children in two groups

story. The adult guided play (encouraged engaged in reading \& play

Weisberg
2015 USA $154 \quad$ 4-6 RCT, pre-post $\begin{gathered}\text { School/ } \\ \text { classroom }\end{gathered}$ Group Researcher 6-10 $\quad \begin{gathered}\text { Figurines/ } \\ \text { toy props }\end{gathered}$

den-ended questions,

sessions, but with either

realistic (e.g., farm) or

Literacy/

used/defined target words). See

fantasy (e.g., dragon) story

language

comparison to right for more information

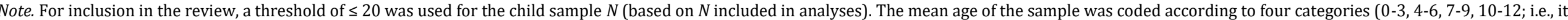

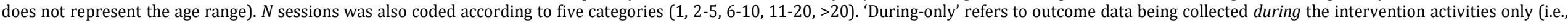

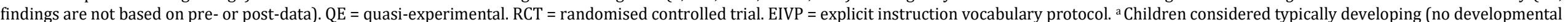

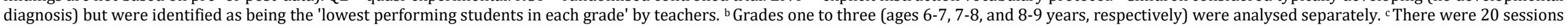

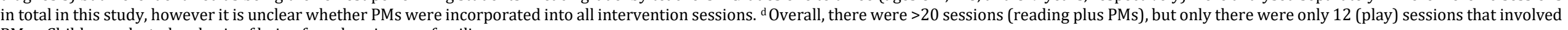
PMs. ${ }^{e}$ Children selected on basis of being from low-income families. 


\subsubsection{Science-based PMs (n = 9)}

\section{KEY FINDINGS}

- Intervention activities, materials, \& knowledge being learned varied substantially, from experimenting with items to learn about gravity or magnetism, to building simple \& complex machines to learn early engineering \& physics-related skills.

- The development of interventions designed to introduce young children to complex scientific concepts, through playful activities that promote active participation, is encouraging, but more research is needed.

\section{Overview}

Children taking part in the science-based interventions engaged in hands-on experimentation with different materials. Activities related to numerous scientific concepts such as speed and slope [van Schijndel], falling objects [Lazonder]), balance beam and mass [Zacharia], natural science (dinosaurs and fossils [Lu]), simple machines (related to engineering principles and physics; [Li; Marulcu; Portsmore]), and gravity, buoyancy, magnets, and air [Bulunuz; Dejonckheere]. The level of playfulness and the degree of physical manipulation that children engaged in within these interventions ranged from low to high. In some children could freely experiment with materials or create their own solutions in engineering tasks, while being supported by an adult. Others involved more constrained activities with specific materials and prescribed actions. See Table 4 for a summary of study characteristics.

\begin{tabular}{|c|c|}
\hline \multicolumn{2}{|c|}{ KEY INTERVENTION FEATURES } \\
\hline Location & $\begin{array}{l}\text { USA (2), Netherlands (2), Belgium (1), China (1), } \\
\text { Cyprus (1), Taiwan (1), Turkey (1) }\end{array}$ \\
\hline Setting & $\begin{array}{l}\text { School/classroom (7), childcare centre (1), } \\
\text { unclear (1) }\end{array}$ \\
\hline Adult involved & Teacher (5), researcher (3), unclear (1) \\
\hline Main outcome domains & Science-based (7), exploratory play (2) \\
\hline
\end{tabular}

\section{Interventions}

Most interventions involved high levels of physical engagement. In three studies children used complex LEGO engineering materials to create simple machines (i.e., a mechanical device that changes the direction or magnitude of force, like a lever or pulley; [Li; Marulcu; Portsmore]). In other studies children played in a sandpit and interacted with slope- and speed-related items (e.g., tubes and small objects; [van Schijndel]), or actively engaged in hands-on activities related to scientific concepts (e.g., testing whether objects float or sink, playing with magnets, learning about gravity by dropping items [Bulunuz; Dejonckheere]). The level of physical manipulation was relatively limited in the three remaining studies in which children simply dropped balls from different heights (to gain an 
understanding of how factors such as mass, size, and height may or may not impact how fast items fall; [Lazonder]), or held/placed items on a balance beam [Zacharia]. In another study children used hand-outs to work out how to place triangle pieces (printed with fossils and dinosaur names) next to corresponding hexagons (with dinosaur outlines; [Lu]). In all three of these studies, physical materials were compared to computerised tasks.

Three studies were conceptualised as being play-based - affording children freedom within the hands-on activities, with guidance and scaffolding from a teacher. Two of these were curriculumbased or integrated into an existing curriculum [Bulunuz; van Schijndel]. In one, children could interact with different learning stations related to science concepts such as magnets, gravity, and water [Bulunuz]. Children could freely explore and experiment with various materials and engaged in problem-solving activities set by the teacher (e.g., removing paperclips from a glass of water without touching them at the magnet station). In the other study, play objects were provided in sandpits (e.g., plastic tubes and small objects to learn about slope and speed; [van Schijndel]). Children were encouraged to participate in the sandpit at least once a week but were free to join in as often as they liked. In the third study, teachers provided science-based materials, demonstrated their use (e.g., magnets, objects that float/sink), and then encouraged and scaffolded children's exploratory play with the materials [Dejonckheere]. However, the number of intervention sessions in this study was unclear. Studies involving LEGO engineering (i.e., making simple and/or complex machines) were not explicitly described as play-based, but the interventions could be characterised as guided play as children were set a challenge, worked iteratively in collaboration with peers to design and make a solution to the problem, and were assisted/guided by an adult [Li; Marulcu; Portsmore]. In another study children played a game with rules [Lu]. Two studies were not playbased and employed a didactic approach in which children had limited choice in the intervention activities [Lazonder; Zacharia].

The rationale for incorporating PMs in science-based interventions varied across studies. For younger pre-primary children, hands-on learning was used to teach science through play, and to encourage their scientific reasoning by tapping into their intrinsic motivation to explore the world [Bulunuz; van Schijndel]. For older primary-age children, LEGO-based simple machine problems were used to increase their exposure to engineering principles [Li; Marulcu; Portsmore]. The concrete-toabstract theory is also mentioned in several papers [Bulunuz; Lazonder; Zacharia] (see also sections 2.5.2 and 2.5.3), some of which examined whether physical manipulation of tangible objects was important [Lazonder; Zacharia].

\section{Evidence relating to effectiveness}

Some positive effects were reported in studies using a didactic approach for teaching specific knowledge (within a constrained activity). In one, children's false beliefs about mass and size were assessed before and after taking part in a dropping experiment [Lazonder]. Those who engaged in a physical dropping task were more likely to revise their misconceptions about mass than those who engaged in a virtual dropping task or simply watched an adult drop objects. However, there were no group differences in children's likelihood of correcting false beliefs about size. In another study, children's understanding of balance beams was assessed before and after taking part in a physical or computerised balance beam task (children with higher and lower levels of 'correct prior knowledge' about balance beams were analysed independently) [Zacharia]. There was no differential impact of 
intervention type on children who had correct prior knowledge. Conversely, children with incorrect prior knowledge in the PM group improved (and caught up to those with correct prior knowledge), while those in the virtual condition did not. In contrast, children learned more dinosaur-related knowledge by playing a virtual (augmented reality) game than a physical paper-based version [Lu]. However, these studies are limited: children only engaged in a single intervention session, the tasks were highly constrained, and the authors did not explore generalisation of effects beyond specific target knowledge.

Positive effects were reported by two studies involving playful hands-on experimentation [Bulunuz; van Schijndel]. Children who took part in a guided play curriculum involving science-based learning stations outperformed those taught using a didactic approach on measure of science knowledge [Bulunuz]. However, outcome data were derived from interview questions that mostly relied on children recalling their intervention experiences and the potential generalisation of learning to novel scenarios was not assessed. Therefore, the effects may not reflect improvements in children's conceptual understanding. In the other study, children engaged in more exploratory play following a guided play sandpit intervention than free play controls [van Schijndel]. Both studies were limited by small samples (both $\mathrm{Ns}<30$ ).

Finally, the effects for interventions involving LEGO-based engineering, in which children followed a design process to make simple machines, were mixed. In two of these studies, children took part in the LEGO-engineering programme with or without a design planning stage prior to building. Overall (independent of group), children showed gains on a physics measure [Li] and in their ability to solve a novel design problem with familiar (LEGO), but not novel (non-LEGO), materials [Portsmore]. However, a lack of group differences indicated that there was no additive benefit of the design planning stage. In another study, the programme was compared to an alternative (non-LEGO-based), but still hands-on, inquiry-based approach for learning about levers and pulleys [Marulcu]. The LEGO-based group outperformed controls on an interview-based learning measure of simple machines, but not on a written task. These studies are limited by small samples (all $N s<32$ ), and lacked additional comparison conditions (e.g., without PMs), thus limiting the conclusions that can be drawn (e.g., to determine whether the physical nature of the tasks is important). Further details regarding the findings of each study can be found in Appendix D (section 7.4.4).

\section{Summary}

\section{Key findings}

Overall, there were some positive findings reported for the effects of science-based studies involving hands-on learning and experimentation. The interventions were implemented across a wide age range: from younger (0-3 years) to older (10-12 years) children. An encouraging observation is that interventions targeting complex scientific concepts such as engineering and physics, and promote playfulness, creativity, collaboration, and children's active engagement in learning, can be delivered with primary school-age children [Li; Marulcu; Portsmore]. This body of literature also demonstrates that pre-primary children can be introduced to, and start learning about, scientific concepts from as young as two and three years old through playful exploration and experimentation with hands-on materials [Bulunuz]. 
Key gaps \& recommendations for future research

- All the studies were conducted in upper-middle- or high-income contexts, with none taking place in lower-income countries. The field also lacks large-scale intervention studies, with those identified all having small sample sizes (all $N s<100$ ). There is a need for more geographically diverse research and larger-scale effectiveness trials.

- Outcome measures were typically developed by the authors and involved content that overlapped substantially with the intervention tasks. Research is needed to establish whether intervention-related benefits are transferable; that is, to examine if children can generalise their learning experiences to novel settings, and if not, to consider how adults can scaffold hands-on science activities so that children gain a broader conceptual understanding beyond the parameters of the intervention tasks.

- A relatively small number of science-based studies were identified ( $n=9)$, despite there being a potentially vast array of materials and objects that could be used for hands-on science learning 27 . A limitation of this review is that the search terms do not exhaustively capture the wide range of physical materials used in educational interventions. While manipulative is frequently used to describe tangible objects used in math activities, the term is not ascribed to physical materials as often in other learning domains such as science. A diverse range of materials were identified, such as magnets, parachutes, balls, a sandpit, plastic tubes, water and ice, balance beams, and so on. A more targeted search (i.e., with search terms relating to specific science-based materials) may yield additional relevant reports involving sciencebased PMs.

${ }^{27}$ Note that numerous other studies, that tested whether interventions involving LEGO-based simple machines and other complex LEGO kits (e.g., robotics programmes) promote primary-age children's STEM-learning, were also identified in the search, but were excluded due to them involving electronic materials. 
PEDAL Report: PM scoping review

Table 4. Characteristics of studies involving science-based PMs $(n=9)$.

\begin{tabular}{|c|c|c|c|c|c|c|c|c|c|c|c|c|}
\hline ID & Location & $\begin{array}{c}\text { Sample } \\
\qquad N\end{array}$ & Age & $\begin{array}{l}\text { Research } \\
\text { design }\end{array}$ & Setting & $\begin{array}{l}\text { Model of } \\
\text { delivery }\end{array}$ & $\begin{array}{l}\text { Adult } \\
\text { involved }\end{array}$ & $\begin{array}{c}N \\
\text { sessions }\end{array}$ & Materials & Intervention & Comparison(s) & $\begin{array}{c}\text { Main } \\
\text { outcome } \\
\text { domains }\end{array}$ \\
\hline $\begin{array}{l}\text { Bulunuz } \\
2013\end{array}$ & Turkey & 26 & $4-6$ & $\begin{array}{l}\text { QE, pre- } \\
\text { post }\end{array}$ & $\begin{array}{l}\text { School/ } \\
\text { classroom }\end{array}$ & $\begin{array}{l}\text { Class/ } \\
\text { group }\end{array}$ & Teacher & $>20$ & $\begin{array}{l}\text { Science-based } \\
\text { materials (e.g., } \\
\text { magnets, toy } \\
\text { parachute, } \\
\text { water/ice) }\end{array}$ & $\begin{array}{l}\text { Curriculum approach (for learning science } \\
\text { concepts like gravity) involving playful } \\
\text { hands-on activities/experiments (e.g., } \\
\text { mixing colours, using magnets; some were } \\
\text { not hands-on, e.g., watching seeds grow) } \\
\text { with adult support (questions, modelling) }\end{array}$ & $\begin{array}{l}\text { Didactic approach } \\
\text { (without hands-on } \\
\text { activities) }\end{array}$ & $\begin{array}{c}\text { Science- } \\
\text { based }\end{array}$ \\
\hline $\begin{array}{l}\text { Dejonckh- } \\
\text { eere } \\
2016\end{array}$ & Belgium & 57 & $4-6$ & $\begin{array}{l}\text { RCT, pre- } \\
\text { post }\end{array}$ & $\begin{array}{l}\text { School/ } \\
\text { classroom }\end{array}$ & $\begin{array}{l}\text { Class/ } \\
\text { group }\end{array}$ & Teacher & Unclear a & $\begin{array}{l}\text { Science-based } \\
\text { materials (e.g., } \\
\text { magnets, objects } \\
\text { that float/sink) }\end{array}$ & $\begin{array}{l}\text { Exploratory play with science-based } \\
\text { materials (e.g., magnets, objects that float } \\
\text { or sink, balance scale) and adult guidance }\end{array}$ & Business-as-usual & $\begin{array}{l}\text { Play- } \\
\text { based }\end{array}$ \\
\hline $\begin{array}{l}\text { Lazonder } \\
2014\end{array}$ & Netherlands & 60 & $\begin{array}{l}10- \\
12\end{array}$ & $\begin{array}{l}\text { RCT, pre- } \\
\text { post }\end{array}$ & Unclear & Individual & Researcher & 1 & $\begin{array}{l}\text { Light \& heavy } \\
\text { tennis, soccer, \& } \\
\text { ping-pong balls }\end{array}$ & $\begin{array}{l}\text { Children dropped different balls from } 1 \mathrm{~m} \\
\text { or } 2 \mathrm{~m} \text { \& made predictions about which } \\
\text { would hit the ground first }\end{array}$ & $\begin{array}{l}\text { 1. Computerised } \\
\text { drop task } \\
\text { 2. Adult dropped } \\
\text { (child observed) }\end{array}$ & $\begin{array}{l}\text { Science- } \\
\text { based }\end{array}$ \\
\hline $\begin{array}{c}\mathrm{Li} \\
2016\end{array}$ & China & 30 & $\begin{array}{l}10- \\
12\end{array}$ & $\begin{array}{l}\text { RCT, pre- } \\
\text { post }\end{array}$ & $\begin{array}{l}\text { School/ } \\
\text { classroom }\end{array}$ & Group & Teacher & $2-5$ & $\begin{array}{l}\text { LEGO engineering } \\
\text { materials }\end{array}$ & $\begin{array}{l}\text { Children collaborated to design \& make } \\
\text { 'simple machines' (e.g., crane, fan). } \\
\text { Teachers guided a step-by-step design } \\
\text { process: identify problem, develop \& } \\
\text { choose solution, build \& test }\end{array}$ & $\begin{array}{l}\text { Same as } \\
\text { intervention } \\
\text { without the design } \\
\text { process }\end{array}$ & $\begin{array}{l}\text { Science- } \\
\text { based }\end{array}$ \\
\hline $\begin{array}{c}\mathrm{Lu} \\
2018\end{array}$ & Taiwan & 53 & $\begin{array}{c}10- \\
12\end{array}$ & $\begin{array}{l}\text { QE, pre- } \\
\text { post }\end{array}$ & $\begin{array}{l}\text { School/ } \\
\text { classroom }\end{array}$ & Group & Unclear & 1 & Card puzzle & $\begin{array}{l}\text { Physical puzzle \& hand-outs: children } \\
\text { used paper-based hand-outs to help them } \\
\text { match hexagonal dinosaur pieces with } \\
\text { associated triangular pieces (fossils \& } \\
\text { dinosaur names) }\end{array}$ & $\begin{array}{l}\text { Physical puzzle \& } \\
\text { virtual guidance } \\
\text { (multimedia } \\
\text { content) }\end{array}$ & $\begin{array}{l}\text { Science- } \\
\text { based }\end{array}$ \\
\hline $\begin{array}{l}\text { Marulcu } \\
2016\end{array}$ & USA & 31 & $\begin{array}{l}10- \\
12\end{array}$ & $\begin{array}{l}\text { QE, pre- } \\
\text { post }\end{array}$ & $\begin{array}{l}\text { School/ } \\
\text { classroom }\end{array}$ & $\begin{array}{l}\text { Class/ } \\
\text { pairs }\end{array}$ & Teacher & $11-12$ & $\begin{array}{l}\text { LEGO engineering } \\
\text { materials }\end{array}$ & $\begin{array}{l}\text { Children collaborated to plan \& make a } \\
\text { 'simple machine' ('people mover') using a } \\
\text { design process: identify problem, develop } \\
\text { \& choose solution, build \& test }\end{array}$ & $\begin{array}{l}\text { Hands-on inquiry- } \\
\text { based learning } \\
\text { about levers \& } \\
\text { pulleys }\end{array}$ & $\begin{array}{l}\text { Science- } \\
\text { based }\end{array}$ \\
\hline $\begin{array}{l}\text { Portsmore } \\
2010\end{array}$ & USA & 24 & $7-9$ & $\begin{array}{l}\text { QE, pre- } \\
\text { post }\end{array}$ & $\begin{array}{l}\text { School/ } \\
\text { classroom }\end{array}$ & Class & Researcher & $>20$ & $\begin{array}{l}\text { LEGO engineering } \\
\text { materials }\end{array}$ & $\begin{array}{l}\text { LEGO-engineering curriculum \& story } \\
\text { (Goldilocks): children planned, drew, \& } \\
\text { built a solution to a design problem, with } \\
\text { support from a teacher who set the } \\
\text { challenge \& asked questions }\end{array}$ & $\begin{array}{l}\text { Spontaneous LEGO- } \\
\text { engineering (same } \\
\text { as intervention } \\
\text { without planning } \\
\text { stage) }\end{array}$ & $\begin{array}{l}\text { Science- } \\
\text { based }\end{array}$ \\
\hline $\begin{array}{l}\text { van } \\
\text { Schijndel } \\
2010\end{array}$ & Netherlands & 28 & $0-3$ & $\begin{array}{l}\text { QE, pre- } \\
\text { post }\end{array}$ & $\begin{array}{l}\text { Childcare } \\
\text { centre }\end{array}$ & Group & Teacher & $6-10 \mathrm{~b}$ & $\begin{array}{l}\text { Sandpit \& various } \\
\text { items (e.g., bucket, } \\
\text { ball, ball, sieve, } \\
\text { tubes) }\end{array}$ & $\begin{array}{l}\text { Guided-play in sandpit with science-based } \\
\text { materials to learn about slope \& speed, \& } \\
\text { sorting \& sets. The teacher supported } \\
\text { children's exploration/experimentation } \\
\text { (questioning, modelling use of items) }\end{array}$ & $\begin{array}{l}\text { Free play in sandpit } \\
\text { with same materials }\end{array}$ & $\begin{array}{l}\text { Play- } \\
\text { based }\end{array}$ \\
\hline
\end{tabular}


PEDAL Report: PM scoping review

2012

Cyprus

80

4-6 QE, pre-

School/

classroom

Individual Researcher

various objects

(e.g., toys, cubes)

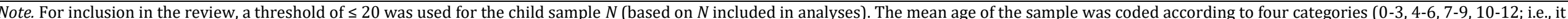

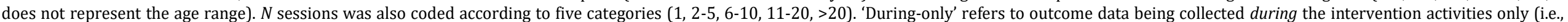

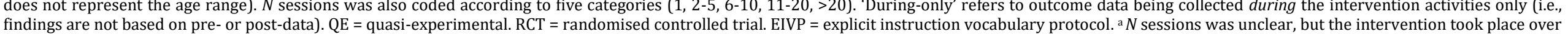
seven weeks. ${ }^{\mathrm{b}}$ The programme lasted six weeks during regular playtime; children were encouraged to participate at least once a week, but were free to join as often as they liked.
Computerised

Sciencebeam 


\subsubsection{Paper folding $(n=7)$}

\section{KEY FINDINGS}

- Children engaged in paper folding activities - that were typically highly prescribed \& adult directed - to create 3D objects.

- There were some limited findings that paper folding activities benefit children's math ability \& spatial/visualisation skills, but overall, the evidence was unconvincing.

\section{Overview}

This section summarises interventions involving paper folding (origami) and paper engineering activities (i.e., transforming paper into 3D objects). The degree of physical engagement for this group of studies is deemed high, as they all involved children creating something out of the materials (e.g., 3D geometric shapes or animals). None of the studies were explicitly described as being play-based and mostly involved didactic teaching methods. Still, the degree of freedom that children had within the intervention activities varied between studies, with some being more aligned with guided play approaches. All were conducted in high-income contexts except one that took place in a middleincome country [Cakmak]. None were delivered in lower-income countries.

\begin{tabular}{|c|c|}
\hline \multicolumn{2}{|c|}{ KEY INTERVENTION FEATURES } \\
\hline Location & USA (4), Hungary (1), Portugal (1), Turkey (1) \\
\hline Setting & School/classroom (7) \\
\hline Adult involved & $\begin{array}{l}\text { Researcher (4), teacher/TA (2), } \\
\text { other [school psychologist] (1) }\end{array}$ \\
\hline Main outcome domains & $\begin{array}{l}\text { Visual-spatial (5), math-spatial (2), } \\
\text { math/numeracy (2), social-emotional (1) }\end{array}$ \\
\hline
\end{tabular}

\section{Interventions}

For a summary of intervention characteristics, see Table 5. Didactic instruction was typically used during the paper folding activities. For example, an adult would provide step-by-step instructions and model each folding action [Boakes; Cakmak; Yuzawa] or children would follow pre-printed patterns [Krisztián] ${ }^{28}$. In one study, the adult even interrupted a child if they folded the paper improperly and helped them to refold it [Yuzawa]. In some studies, the adult would also intersperse relevant geometric terms [Boakes] or encourage the class to discuss shape properties and math terms encountered during paper folding [Cakmak]. Despite the limited degree of child choice within the folding activity, children in one study had relatively more freedom for creative expression when

${ }^{28}$ These structured paper-folding activities resemble the structured block building activities described earlier in the report (see section 2.5.1). 
they later decorated their 3D model (e.g., drawing on an octahedron to create a mouse or dog; [Krisztián]).

Two studies investigated the Think3d! origami and pop-up paper engineering programme [Burte; Taylor]. Children completed challenges that required them to visualise, plan, and construct 3D paper models. The intervention activities were not prescriptive and provided children with opportunities for exploration, iteration, and collaboration with their peers, with guidance from an adult. Although the authors did not ascribe the term guided play to the Think3d! programme, it is more aligned with a play-based than didactic approach and was described as playful and engaging by the authors [Taylor]. One remaining study did not provide enough information about the origami programme to determine the level of adult guidance and child autonomy [Raimundo] ${ }^{29}$.

\section{Evidence relating to effectiveness}

Most of the paper folding studies had one or more methodological shortcomings, including small sample sizes ( $n$ s < 100; [Boakes; Burte; Cakmak; Krisztián; Taylor; Yuzawa]), lack of a transfer measures (i.e., outcome data was based on performance during the intervention; [Yuzawa]), passive control groups [Boakes; Krisztián], or lack of comparison conditions altogether [Burte; Cakmak]. One study had a more rigorous research design, including a larger sample size $(n=318)$ and the inclusion of an active comparison condition [Raimundo]. However, the findings of this paper do not hold much weight in the context of the review, as it examined the impact of a social-emotional intervention for enhancing children's social-emotional competencies, with an origami curriculum acting as the control condition.

Key findings have been grouped according to four outcome domains that emerged: math/numeracy, math-spatial, visual-spatial, and social-emotional. See Appendix D (section 7.4.5) for an overview of the main findings of each study. There was weak evidence that paper folding interventions benefited children's math ability: an origami programme resulted in larger gains than a passive control condition, however this effect was based on an interaction effect that was not followed-up with pairwise comparisons [Krisztián], and there were marginal gains for older (grades five and six) but not younger (grades three and four) children who took part in the Think3d! paper engineering programme, however the study lacked a comparison condition [Burte]. There was also limited evidence for benefits to math-spatial skills. Children did better on size comparison tasks when they also completed origami tasks, as compared to children who only completed size comparison tasks [Yuzawa]. However, origami-math lessons had no impact on children's geometry knowledge [Boakes].

Children's spatial ability was typically measured using 2D and 3D spatial visualisation and transformation tasks (e.g., mental rotation, mental knotting, mental paper folding, surface development, make-a-die). Effects in this domain were mixed and inconclusive: overall, three studies reported null effects to spatial visualisation tasks following paper folding interventions [Boakes; Burte; Taylor], two reported gains in spatial ability relative to passive controls (though these effects were only found for $t$-test comparisons; [Krisztián; Taylor]), and although another two demonstrated improvements in spatial visualisation skills, they both lacked comparison conditions, meaning the

\footnotetext{
${ }^{29}$ In this study, the origami curriculum acted as an active control condition for a social-emotional programme that was being delivered.
} 
gains could simply reflect practice or Hawthorne effects [Burte; Cakmak]. Finally, an origami curriculum did not benefit children's social-emotional competencies relative to a social-emotional learning programme [Raimundo].

\section{Summary}

\section{Key findings}

To summarise, findings were mixed and there was no persuasive evidence that paper folding interventions benefit children's math or visual-spatial skills. This is in part due to methodological shortcomings across the studies, limiting the robustness of reported positive effects.

Key gaps \& recommendations for future research

- All the interventions were implemented in middle- or high-income countries, with none taking place in lower-income contexts. There was also a lack of methodologically rigorous research. Future investigations need to be of higher methodological quality and more geographically diverse.

- Interventions typically involved didactic instruction, with little freedom or creativity afforded to children within the paper folding activities. While there were a couple of studies that implemented a more playful approach (the Think3d! programme), both lacked an active control group and neither provided convincing evidence for the effectiveness of the programme. Future research could further explore the potential benefits of play-based approaches involving paper folding, perhaps when integrated with other types of hands-on PM-based activities. 
PEDAL Report: PM scoping review

Table 5. Characteristics of studies in involving paper folding $(n=7)$.

\begin{tabular}{|c|c|c|c|c|c|c|c|c|c|c|c|c|}
\hline ID & Location & $\begin{array}{c}\text { Sample } \\
\quad N\end{array}$ & Age & $\begin{array}{l}\text { Research } \\
\text { design }\end{array}$ & Setting & $\begin{array}{l}\text { Model of } \\
\text { delivery }\end{array}$ & $\begin{array}{c}\text { Adult } \\
\text { involved }\end{array}$ & $\begin{array}{c}N \\
\text { sessions }\end{array}$ & Materials & Intervention & Comparison(s) & $\begin{array}{l}\text { Main outcome } \\
\text { domains }\end{array}$ \\
\hline $\begin{array}{l}\text { Boakes } \\
2009\end{array}$ & USA & 56 & $\begin{array}{l}10- \\
12\end{array}$ & $\begin{array}{l}\text { QE, pre- } \\
\text { post }\end{array}$ & $\begin{array}{c}\text { School/ } \\
\text { classroom }\end{array}$ & Class & Teacher & $11-20$ & Paper & $\begin{array}{l}\text { Origami-math lessons: step-by-step } \\
\text { instructions on folding origami models, } \\
\text { geometric terms used during }\end{array}$ & No-intervention & $\begin{array}{l}\text { Visual-spatial, } \\
\text { math-spatial }\end{array}$ \\
\hline $\begin{array}{l}\text { Burte } \\
2017\end{array}$ & USA & 86 & $\begin{array}{l}7-9 \\
10- \\
12\end{array}$ & $\begin{array}{l}\text { One } \\
\text { group, } \\
\text { pre-post }\end{array}$ & $\begin{array}{c}\text { School/ } \\
\text { classroom }\end{array}$ & $\begin{array}{l}\text { Class/ } \\
\text { group }\end{array}$ & Teacher & $6-10$ & Paper & $\begin{array}{l}\text { Think3d! programme: origami \& pop-up paper } \\
\text { engineering (cutting \& folding). Teachers } \\
\text { guided children by explaining links between } \\
\text { instructions/models \& materials rather than } \\
\text { explicitly directing them }\end{array}$ & $\begin{array}{l}\text { N/A (no control } \\
\text { group) }\end{array}$ & $\begin{array}{c}\text { Math/numeracy, } \\
\text { visual-spatial }\end{array}$ \\
\hline $\begin{array}{l}\text { Cakmak } \\
2014\end{array}$ & Turkey & 38 & $\begin{array}{l}10- \\
12\end{array}$ & $\begin{array}{l}\text { One } \\
\text { group, } \\
\text { pre-post }\end{array}$ & $\begin{array}{l}\text { School/ } \\
\text { classroom }\end{array}$ & Class & Researcher & $6-10$ & Paper & $\begin{array}{l}\text { The adult modelled origami steps that children } \\
\text { followed, gave instructions, \& monitored them } \\
\text { (repeating steps if needed). The whole class } \\
\text { also discussed geometric \& math concepts } \\
\text { encountered in the activity }\end{array}$ & $\begin{array}{l}\text { N/A (no control } \\
\text { group) }\end{array}$ & Visual-spatial \\
\hline $\begin{array}{l}\text { Krisztián } \\
2015\end{array}$ & Hungary & $37 \mathrm{a}$ & $\begin{array}{l}10- \\
12\end{array}$ & $\begin{array}{l}\text { RCT, pre- } \\
\text { post }\end{array}$ & $\begin{array}{l}\text { School/ } \\
\text { classroom }\end{array}$ & Group & Researcher & $6-10$ & Paper & $\begin{array}{l}\text { Origami training: children (with math } \\
\text { difficulties) used pre-printed patterns (also } \\
\text { cutting, pasting, decorating creations), the } \\
\text { tasks increased in difficulty over the sessions }\end{array}$ & $\begin{array}{l}\text { No-intervention } \\
\text { (children with \& } \\
\text { without math } \\
\text { difficulties) }\end{array}$ & $\begin{array}{c}\text { Math/numeracy, } \\
\text { visual-spatial }\end{array}$ \\
\hline $\begin{array}{l}\text { Raimundo } \\
2013\end{array}$ & Portugal & 318 & $7-9$ & $\begin{array}{l}\text { QE, pre- } \\
\text { post }\end{array}$ & $\begin{array}{c}\text { School/ } \\
\text { classroom }\end{array}$ & Unclear & $\begin{array}{l}\text { Other } \\
\text { (school } \\
\text { psychologist) }\end{array}$ & $>20$ & Paper & Origami/paper folding curriculum & $\begin{array}{c}\text { Social-emotional } \\
\text { learning programme }\end{array}$ & $\begin{array}{l}\text { Social- } \\
\text { emotional }\end{array}$ \\
\hline $\begin{array}{l}\text { Taylor } \\
2013\end{array}$ & USA & 39 & $\begin{array}{l}10- \\
12\end{array}$ & $\begin{array}{l}\text { QE, pre- } \\
\text { post }\end{array}$ & $\begin{array}{l}\text { School/ } \\
\text { classroom }\end{array}$ & $\begin{array}{l}\text { Class/ } \\
\text { group }\end{array}$ & Researcher & $6-10$ & Paper & $\begin{array}{l}\text { The Think3d! programme: origami \& pop-up } \\
\text { paper engineering. Lessons included diagrams } \\
\text { \& instructions, \& challenged children to figure } \\
\text { out how to complete models }\end{array}$ & No-intervention & Visual-spatial \\
\hline $\begin{array}{c}\text { Yuzawa } \\
2002\end{array}$ & USA & 24 & $4-6$ & $\begin{array}{l}\text { RCT, } \\
\text { during- } \\
\text { only }\end{array}$ & $\begin{array}{c}\text { School/ } \\
\text { classroom }\end{array}$ & Individual & Researcher & $2-5$ & Paper & $\begin{array}{l}\text { Origami \& size comparison tasks. The adult } \\
\text { modelled paper folding steps. If a child made a } \\
\text { mistake the adult stopped \& corrected them }\end{array}$ & $\begin{array}{l}\text { Size comparison } \\
\text { tasks only }\end{array}$ & Math-spatial \\
\hline
\end{tabular}




\subsubsection{Puzzles \& Tangrams $(n=6)$}

\section{KEY FINDINGS}

- There was some limited evidence that Tangram (shape-based) puzzle tasks benefited children's geometry skills.

- Evidence suggests that virtual materials were just as good, or better than, PMs.

\section{Overview}

This section summarises puzzle-based interventions in which children arranged different shapes to produce target configurations. The types of puzzle activities varied across studies ${ }^{30}$, as did the level of physical engagement and child choice afforded within the activities. Most interventions involved geometric shapes ${ }^{31}$, with children completing multiple tasks (e.g., solving shape-based puzzles, combining shapes into collages) or Tangram ${ }^{32}$ activities to learn geometry [Casey 2008b, 2008c; Olkun; Thompson 2016]. In the others, children used puzzle-based maps to learn about geographical locations [Dang; Eisen]. See Table 6 for an overview of study characteristics.

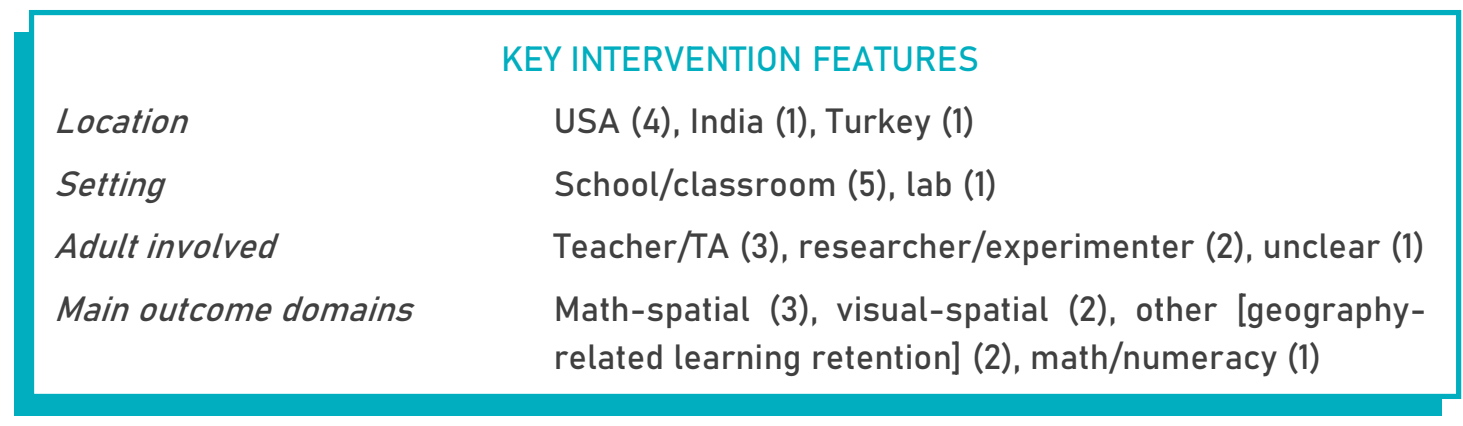

\section{Interventions}

Children engaged in low to moderate levels of physical manipulation with the materials. In some studies, the puzzle activities provided limited opportunities for physical engagement due to the restricted way that puzzle pieces could be arranged; in two studies children placed outlines of geographical locations on puzzle maps [Dang; Eisen]. In other studies, there were many ways that geometric shapes could be arranged, and children could flip or rotate them when creating target patterns or shapes [Casey 2008b \& 2008c; Olkun; Thompson 2016].

Three studies compared physical and virtual puzzle tasks [Eisen; Olkun; Thompson 2016]. For example, children solved Tangram puzzles with wooden pieces - arranging the shapes to match model images (e.g., the outline of a rabbit) - or completed the same puzzles using a tablet computer

\footnotetext{
${ }^{30}$ Note that none of the studies involved traditional jigsaw puzzles.

${ }^{31}$ Studies involving geometry-based puzzle activities are included here but overlap substantially with math manipulative interventions (see section 2.5.2) as they are typically used in math (geometry) instruction.

32 Tangrams are geometric puzzles made up of seven pieces (including a parallelogram, a square, and five triangles of different sizes), which can be arranged in different configurations to create many other shapes with varying degrees of complexity.
} 
[Thompson 2016]. The concrete-to-abstract theory is referred to in one study that examined whether active touch is important when learning with PMs [Olkun]. In another study, children studied world and Asia maps with puzzle-based or non-puzzle maps [Dang].

Two separate experiments investigated the impact of a geometry intervention involving storytelling combined with multiple puzzle-based tasks, compared to business-as-usual or geometry-only (puzzle tasks without storytelling) comparison conditions [Casey 2008b, 2008c]. Although the intervention was not explicitly described as guided play, it could be characterised as such. Children worked collaboratively with their peers to solve challenges set by their teacher and engaged in activities that afforded more freedom and creativity (e.g., exploring different ways of combining triangles to make dragon collages or creating novel configurations). Furthermore, the activities were embedded in a playful and interactive storytelling context - involving puppets, chants, movements, and poems - to motivate and encourage children by providing a meaningful narrative context in which they were helping characters solve problems [Casey, 2008b,2008c].

The other studies were generally not play-based: children were set specific tasks with limited materials and freedom within the activities [e.g., Dang; Olkun]. Teacher guidance was mentioned in one study as a way of helping children discover solutions [Thompson 2016], and another involved a mix of both direct instruction and free play with a puzzle map [Eisen].

\section{Evidence relating to effectiveness}

There was no evidence that physical puzzles were better than computer-based (virtual) activities for improving children's math achievement [Thompson 2016], geometry [Olkun], spatial ability [Thompson 2016], or learning retention (of Australian states) [Eisen]. Furthermore, children who used a puzzle map did worse, or no better, than controls who used a non-puzzle map on a learning retention task of countries and continents [Dang]. Note that most of these studies, with one exception [Thompson 2016], delivered only a single intervention session, and they were further limited by small sample sizes, lack of appropriate pre-test measures, and/or lack of transfer tasks (i.e., a measure of outcome beyond learning retention of intervention task content).

Selective benefits were reported following a storytelling geometry intervention to a triangle task (familiar shapes ${ }^{33}$ ) and a Tangram task (novel shapes) [Casey 2008b; 2008c]. Intervention children outperformed geometry-only [Casey 2008c] and passive controls [Casey 2008b] on the triangle task and had greater gains on the Tangrams task compared geometry-only [Casey 2008b] but not passive controls [Casey 2008c]. Furthermore, girls benefited more than boys from the two geometry interventions, regardless of whether they also received the storytelling element. Further details regarding the main findings of each study can be found in Appendix D (section 7.4.6).

\section{Summary}

\section{Key findings}

Most of the puzzle- and Tangram-based intervention activities aimed to enhance children's geometry and visuospatial skills (therefore overlapping with the math-based PMs category). A couple of studies involved learning geography knowledge (e.g., locations of countries or continents) using puzzle maps

\footnotetext{
${ }^{33}$ I.e., The same type of triangle shapes that children used in the intervention tasks.
} 
- therefore arguably also tapping children's visuospatial ability. Overall, most studies found that physical puzzle-based tasks were no better, or worse, than virtual activities (or a non-puzzle-based map exercise) for improving children's learning. Selective benefits were reported following a playful intervention involving multiple puzzle-based tasks with geometric shapes, providing some evidence that the programme was better than a passive control condition for improving geometry skills [Casey 2008b; 2008c], but further research would be needed to establish whether it is more effective than a geometry condition without PMs.

Key gaps \& recommendations for future research

- Almost all the studies were conducted in high-income contexts, except one that took place in India, a lower-middle-income country. As with all the previous study categories, this highlights the need for future work that is more geographically diverse.

- Also of note is the lack of curriculum-based interventions: all comprised $\leq$ eight sessions, half of which were limited to only a single exposure; more intensive multi-session programmes may yield more promising findings.

- Furthermore, in line with the pattern that has emerged across all interventions in the review, there is a pressing need for more methodologically rigorous research. 
PEDAL Report: PM scoping review

Table 6. Characteristics of studies in involving puzzles $(n=6)$.

\begin{tabular}{|c|c|c|c|c|c|c|c|c|c|c|c|c|}
\hline ID & Location & $\begin{array}{c}\text { Sample } \\
\quad N\end{array}$ & Age & $\begin{array}{c}\text { Research } \\
\text { design }\end{array}$ & Setting & $\begin{array}{l}\text { Model of } \\
\text { delivery }\end{array}$ & $\begin{array}{c}\text { Adult } \\
\text { involved }\end{array}$ & $\begin{array}{c}N \\
\text { sessions }\end{array}$ & Materials & Intervention & Comparison(s) & $\begin{array}{l}\text { Main outcome } \\
\text { domains }\end{array}$ \\
\hline $\begin{array}{l}\text { Casey } \\
2008 b \text { a }\end{array}$ & USA & 155 & $4-6$ & $\begin{array}{l}\text { RCT, pre- } \\
\text { post }\end{array}$ & $\begin{array}{l}\text { School/ } \\
\text { classroom }\end{array}$ & $\begin{array}{l}\text { Class/ } \\
\text { group }\end{array}$ & Teacher & $6-10$ & $\begin{array}{l}\text { 2D \& 3D } \\
\text { geometric } \\
\text { puzzles }\end{array}$ & $\begin{array}{l}\text { Story \& geometry: interactive story (chants, } \\
\text { poems, puppets, movements) \& puzzles (e.g., } \\
\text { combining geometric shapes like triangles) }\end{array}$ & $\begin{array}{l}\text { Business-as- } \\
\text { usual (regular } \\
\text { math classes) }\end{array}$ & Math-spatial \\
\hline $\begin{array}{l}\text { Casey } \\
2008 c^{b}\end{array}$ & USA & 63 & $4-6$ & $\begin{array}{l}\text { RCT, pre- } \\
\text { post }\end{array}$ & $\begin{array}{c}\text { School/ } \\
\text { classroom }\end{array}$ & $\begin{array}{l}\text { Class/ } \\
\text { group }\end{array}$ & Teacher & $6-10$ & As above & As above & $\begin{array}{l}\text { Geometry alone } \\
\text { (no story) }\end{array}$ & Math-spatial \\
\hline $\begin{array}{l}\text { Dang } \\
2018\end{array}$ & India & 40 & $7-9$ & $\begin{array}{l}\text { RCT, post- } \\
\text { only c }\end{array}$ & $\begin{array}{l}\text { School/ } \\
\text { classroom }\end{array}$ & $\begin{array}{l}\text { Individual/ } \\
\text { group }\end{array}$ & Researcher & 1 & $\begin{array}{l}\text { Paper puzzle } \\
\text { maps }\end{array}$ & $\begin{array}{l}\text { Children (alone or in pairs) joined puzzle } \\
\text { pieces to make world \& Asia maps. They also } \\
\text { studied the maps to memorise spatial } \\
\text { locations of countries \& continents }\end{array}$ & $\begin{array}{l}\text { No puzzle, maps } \\
\text { only (alone or in } \\
\text { pairs) }\end{array}$ & $\begin{array}{l}\text { Other (learning } \\
\text { retention - } \\
\text { geography) }\end{array}$ \\
\hline $\begin{array}{l}\text { Eisen } \\
2020\end{array}$ & USA & 96 & $4-6$ & $\begin{array}{l}\text { QE, post- } \\
\text { only }\end{array}$ & Lab & Individual & Researcher & 1 & $\begin{array}{l}\text { Wooden } \\
\text { puzzle } \\
\text { map }\end{array}$ & $\begin{array}{l}\text { Physical puzzle map with adult direction } \\
\text { (pieces of Australian states): the adult pointed } \\
\text { to/labelled/traced shapes \& asked children to } \\
\text { name/place/pick pieces up. Children then } \\
\text { engaged in free play with the puzzle }\end{array}$ & $\begin{array}{l}\text { Virtual/app- } \\
\text { based puzzle } \\
\text { with or without } \\
\text { adult direction }\end{array}$ & $\begin{array}{l}\text { Other (learning } \\
\text { retention - } \\
\text { geography) }\end{array}$ \\
\hline $\begin{array}{l}\text { Olkun } \\
2003\end{array}$ & Turkey & 93 & $\begin{array}{l}10- \\
12\end{array}$ & $\begin{array}{l}\text { QE, pre- } \\
\text { post }\end{array}$ & $\begin{array}{l}\text { School/ } \\
\text { classroom }\end{array}$ & Unclear & Unclear & 1 & $\begin{array}{l}\text { Wooden } \\
\text { Tangram } \\
\text { puzzles }\end{array}$ & $\begin{array}{l}\text { Children solved increasingly complex } \\
\text { Tangram puzzles with wooden shapes } \\
\text { (arranging the shapes to fit in an outline) }\end{array}$ & $\begin{array}{l}\text { 1. Virtual puzzle } \\
\text { 2. Business-as- } \\
\text { usual }\end{array}$ & $\begin{array}{l}\text { Math-spatial, } \\
\text { visual-spatial }\end{array}$ \\
\hline $\begin{array}{l}\text { Thompson } \\
2016\end{array}$ & USA & 61 & $7-9$ & $\begin{array}{l}\text { QE, pre- } \\
\text { post }\end{array}$ & $\begin{array}{l}\text { School/ } \\
\text { classroom }\end{array}$ & $\begin{array}{l}\text { Class/ } \\
\text { group }\end{array}$ & Teacher & $2-5$ & $\begin{array}{l}\text { Wooden } \\
\text { Tangram } \\
\text { puzzles }\end{array}$ & $\begin{array}{c}\text { Children solved physical Tangram puzzles (\& } \\
\text { used worksheets \& engaged in group } \\
\text { discussions) }\end{array}$ & $\begin{array}{l}\text { 1. Virtual puzzle } \\
\text { 2. Multimodal } \\
\text { (physical \& } \\
\text { virtual) }\end{array}$ & $\begin{array}{c}\text { Math/numeracy, } \\
\text { visual-spatial }\end{array}$ \\
\hline
\end{tabular}

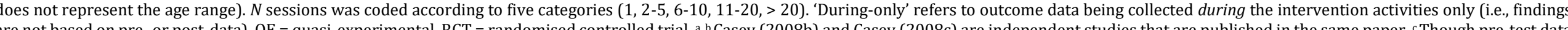

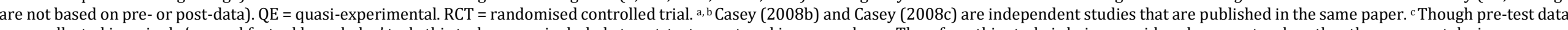

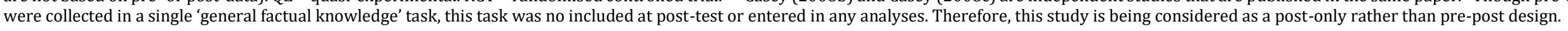




\section{Section 5. Discussion}

\subsection{Overview}

This scoping review sought to identify and map the available evidence on interventions involving PMs for pre-primary- and primary-age children, an important step in identifying gaps for future research. In total, 102 studies were included and were broadly categorised according to the type of intervention materials and/or the main learning domain targeted. This enabled synthesis of a large amount of information. These interventions aimed to improve children's outcomes in a variety of domains, primarily math/numeracy, visual-spatial, language/literacy, and science. Similarities and differences were identified across the included studies that related to the instructional approach used (e.g., didactic or play-based), the types of PMs (e.g., building blocks, figurines, shapes), and the activities that children engaged in (including tasks that required relatively higher or lower degrees of physical engagement, such as construction and paper folding [high], arranging shapes [moderate], or moving a counter [low]). Overall, findings relating to effectiveness of the interventions were mixed, and often confounded by methodological inconsistencies and shortcomings. Within each grouping of studies, there was no consistent evidence that PM-based interventions benefit a particular outcome domain. Nevertheless, there are some positive results to highlight.

\subsection{Key findings}

\section{Evidence related to effectiveness}

Evidence related to effectiveness was mixed. First, there were some reports that block building interventions benefit children's spatial talk, spatial reasoning, and math outcomes. This is consistent with previous research demonstrating associations between early block play, spatial skills, and math (Bower et al., 2020; Mix et al., 2016; Mix \& Cheng, 2012; Verdine, Golinkoff, Hirsh-Pasek, Newcombe, et al., 2014). There were also reports that math-based interventions benefit children's math outcomes, consistent with findings from previous reviews and meta-analytic data (Carbonneau et al., 2013; Holmes, 2013; Lafay et al., 2019). Math-based interventions involving PMs comprised the largest group of studies in the review, likely owing to the well-established use of concrete materials during traditional math instruction. Across the other intervention categories, there were selective benefits to children's language/literacy outcomes following interventions that incorporated PMs in reading/literacy-based activities, to spatial/math skills following puzzle-based activities, and to scientific knowledge through hands-on learning and experimentation with science-based materials.

However, the studies demonstrating positive effects were largely outweighed by additional reports of null, negative, or inconclusive findings. Furthermore, methodological shortcomings and inconsistencies across the studies limited the conclusions that could be drawn regarding the efficacy of PM-based interventions. To assess the strength and quality of the evidence within different learning domains, independent systematic literature reviews are recommended. 
Particularly promising findings were identified for playful curriculum-based interventions, in which children took part in extensive programmes involving math-based PMs (involving $>20$ sessions and numerous activities). In these studies, interventions were compared to passive control groups, meaning that the core components of the interventions could not be determined (e.g., it is unclear if PMs were critical for any observed benefits). Nevertheless, the studies provide evidence for the efficacy of playful, hands-on math-activities that encourage children's active engagement in math learning.

\section{Context}

There was a clear lack of research in lower-income contexts, in fact, over half the studies took place in just one country, namely the USA. Findings reported by these studies are likely not generalisable to different countries and educational systems.

Despite the overwhelming lack of research in lower-income contexts, there were some particularly encouraging findings reported in a large-scale and methodologically rigorous study conducted in Belize - a lower-middle-income country (Hull et al., 2018). In this RCT, the authors found that the math intervention, which involved PMs (plus guidance on how to use them), benefited children's math ability to such a degree that it impacted policy and led to nationwide implementation of the programme. Math instruction in Belize is typically didactic and teacher-centred, with emphasis on memorisation and drill of abstract/symbolic concepts (Hull et al., 2018). The study findings demonstrate how a complex, multifaceted intervention that promotes child-centred learning through hands-on exploration of PMs, can be successfully implemented in a developing context when thoughtful innovations are made to aid implementation and acceptability (e.g., low-cost materials, routine support for teachers). However, it is unclear how individual intervention components contributed to the positive effects.

\section{Level of instructional guidance}

The type of instructional guidance used by the adults delivering the interventions varied across studies, with most employing a guided play or didactic approach. Within some of the studies, the degree of adult guidance and child autonomy was manipulated across intervention conditions. Overall, most studies reported that guided play with PMs was more effective than free play with the same or similar materials for children's visual-spatial, language, and exploratory play outcomes [Borriello; Casey 2008a; Eason; Ferrara; Fisher 2011b, 2013; Toub; van Schijndel], consistent with recent meta-analytic data showing that guided play interventions benefit children's early math skills and spatial talk more than free play (Skene et al., 2021). In the wider literature, there is ongoing debate regarding the optimal level of adult involvement during PM-based math activities, with some evidence that higher levels of guidance supports better learning retention, but that lower levels mediate generalisation of learning (Carbonneau et al., 2013). Future research may further elucidate the differential benefits of higher versus lower levels of adult guidance and child choice during activities with math-based PMs. Furthermore, different children may need different levels of support.

Due to the broad range of interventions captured in this review, the specific effects of guided play with PMs cannot be disentangled from other variables (e.g., differences in the types of materials and/or the amount of physical engagement with PMs). While Skene and colleagues (2021) investigated the effectiveness of guided play interventions (regardless of whether they included PMs 
or not) compared to other types of instruction, and examined potential moderators via subgroup analyses, the type of intervention materials or presence of PMs was not considered. Therefore, further systematic investigation may be warranted to disentangle the relative impact of children's engagement with physical materials and the type of instruction they received.

\section{Level of physical engagement}

The amount of physical engagement that children had with PMs also varied across the intervention activities. For example, block play activities required higher degrees of physical (and visual) object manipulation to place blocks when constructing, whereas other activities simply required children to hold, touch, or move PMs. Prospective studies may provide more insight into the optimal levels of physical engagement for learning with PMs, independent of other factors such as adult guidance.

\section{Physical versus virtual manipulatives}

Overall, there was no convincing evidence that PMs were better than virtual materials for children's learning, with most studies finding that PMs were no better, or worse than, virtual materials. Most of these studies evaluated math interventions, and while the benefits of PMs for math instruction are well-established (Carbonneau et al., 2013; Holmes, 2013; Lafay et al., 2019), the physical nature of the materials used in these interventions may not be critical. Theories of embodied cognition provide insight into the relationship between physical actions and learning: with evidence in other domains such as reading/literacy that imagined or observed manipulation can also be effective, suggesting that children's physical engagement is not essential for learning (Glenberg, 2008; Glenberg et al., 2004). Furthermore, there is neuroscientific evidence that action systems in the brain are activated during literal and observed actions (e.g., Rizzolatti \& Craighero, 2004).

\subsection{Limitations}

Several limitations of this scoping review are outlined. First, is the broad scale/scope of review. While a wide-ranging body of literature was captured, the large number of studies limited the depth of the review. Included studies could only be considered and summarised at a high-level, with potentially valuable information in the reports being disregarded or overlooked (e.g., information relating to theoretical rationale, the level of instructional guidance, the role of play, the importance of physicality, etc). The review aimed to identify educational intervention and programmes using broad inclusion criteria, and studies were included regardless of research design. Consequently, while some studies implemented intensive educational programmes (e.g., that were incorporated into existing curriculums), others were short-term and more experimental in nature (e.g., taking place under strict lab-based conditions that were considerably removed from children's everyday learning experiences). Furthermore, it is difficult to draw conclusions about the implementation of PM-based interventions due to heterogeneity across studies, with great variation in comparison groups, study designs, materials, and outcomes.

There are also numerous limitations related to resource constraints. A single researcher independently conducted most of the review procedure. Consequently, double screening and data extraction of all studies did not take place, and findings may therefore have been susceptible to bias and lack reliability. There were also deviations from the protocol (e.g., there were no searches of the 
grey literature, except for dissertations/theses, or expert consultation). Finally, only reports written in English were included.

\subsection{Recommendations for future research}

Due to the large number and diverse nature of studies included in this review, more targeted examination of the literature may be warranted. For example, the aggregation of data from the most methodologically robust and large-scale trials, may provide more conclusive results regarding the efficacy of PM-based interventions. Furthermore, there may also be value in consolidating the strength and quality of evidence by restricting future reviews to specific learning domains (e.g., reading/literacy) or PM materials of interest (e.g., building blocks).

Additionally, future work is needed to understand the core components of successful interventions, especially those that are multifaceted. A classroom-based intervention may involve multiple components, for example, PMs, hands-on activities, daily practice, sensitive adult guidance, and teacher training/professional development. As the components are intertwined, the relative benefit or necessity of a single component cannot be determined unless multiple comparison groups - with manipulations of certain intervention features - are employed. However, due to challenges associated with implementing complex interventions in low-income contexts, such as funding, teacher training, policy needs, and stakeholder expectations, intervention studies in these countries must rely on fully conceived programmes that have an existing evidence base of success elsewhere (Hull et al., 2018; UNICEF, 2015).

Finally, there is pressing need for further research in lower-income countries. Several recommendations are made for this work:

- Implement and scale interventions that have an established evidence base, or design interventions with components that have pre-existing evidence for their efficacy.

- Design multifaceted interventions that meet context-specific needs.

- Consider adaptations needed so that interventions are useful, acceptable, and feasible for the context - accounting for factors such as existing teacher training (or lack thereof), typical teaching practice, stakeholder expectations, and cost/funding.

- Ensure that adequate support is provided to teachers and/or other personal delivering interventions.

- Conduct studies that are of the highest methodological standard to ensure that any data related to effectiveness is reliable and robust. Better and stronger evidence will give interventions that are deemed effective the best chance of being scaled and impacting policy.

- Encourage transparency and accountability during the development and implementation of interventions. Prior to delivery, develop a comprehensive protocol outlining pre-existing evidence, research questions and/or how the intervention will address context-specific needs, study methodology, and an analysis plan. Include procedures for monitoring adherence. 


\section{Section 6. References}

Alfieri, L., Brooks, P. J., Aldrich, N. J., \& Tenenbaum, H. R. (2011). Does Discovery-Based Instruction Enhance Learning? Journal of Educational Psychology, 103(1), 1-18. https://doi.org/https://doi.org/10.1037/a0021017

Arksey, H., \& O'Malley, L. (2005). Scoping studies: Towards a methodological framework. International Journal of Social Research Methodology: Theory and Practice, 8(1), 19-32. https://doi.org/10.1080/1364557032000119616

Battista, M. T. (1990). Spatial Visualization and Gender Differences in High School Geometry. Journal for Research in Mathematics Education, 21(1), 47. https://doi.org/10.2307/749456

Botha, M., Maree, J. G., \& de Witt, M. W. (2005). Developing and piloting the planning for facilitating mathematical processes and strategies for preschool learners. Early Child Development and Care, 175(7-8), 697-717. https://doi.org/10.1080/0300443042000302582

Bouck, E. C., \& Park, J. (2018). A Systematic Review of the Literature on Mathematics Manipulatives to Support Students with Disabilities. Education and Treatment of Children, 41(1), 65-106. https://doi.org/10.4324/9780203416761_chapter_8

Bower, C., Odean, R., Verdine, B. N., Medford, J. R., Marzouk, M., Golinkoff, R. M., \& Hirsh-Pasek, K. (2020). Associations of 3-year-olds' Block-building Complexity with Later Spatial and Mathematical Skills. Journal of Cognition and Development, 21(3), 383-405. https://doi.org/10.1080/15248372.2020.1741363

Byrne, E. M., Gilbert, R. A., Kievit, R., \& Holmes, J. (2019). Evidence for separate backward recall and n-back working memory factors: A large-scale latent variable analysis. PsyArXiv Pre-Print. https://doi.org/10.31234/osf.io/bkja7

Caldera, Y. M., Culp, A. M. D., O’Brien, M., Truglio, R. T., Alvarez, M., \& Huston, A. C. (1999). Children's play preferences, construction play with blocks, and visual-spatial skills: Are they related? International Journal of Behavioral Development, 23(4), 855-872. https://doi.org/10.1080/016502599383577

Carbonneau, K. J., Marley, S. C., \& Selig, J. P. (2013). A meta-analysis of the efficacy of teaching mathematics with concrete manipulatives. Journal of Educational Psychology, 105(2), 380-400. https://doi.org/10.1037/a0031084

Casey, B. M., Pezaris, E. E., \& Bassi, J. (2012). Adolescent boys' and girls' block constructions differ in structural balance: A block-building characteristic related to math achievement. Learning and Individual Differences, 22(1), 25-36. https://doi.org/10.1016/j.lindif.2011.11.008

Charlesworth, R., \& Lind, K. K. (2003). Math and science for young children (4th ed.). Delmar.

Cheyne, J. A., \& Rubin, K. H. (1983). Playful precursors of problem solving in preschoolers. Developmental Psychology, 19(4). https://doi.org/10.1037/0012-1649.19.4.577

Clements, D. H., \& Sarama, J. (2008). Experimental evaluation of the effects of a research-based preschool mathematics curriculum. American Educational Research Journal, 45(2), 443-494. https://doi.org/10.3102/0002831207312908

Cohen, H. G. (1984). The effects of two teaching strategies utilizing manipulatives on the development of logical thought. Journal of Research in Science Teaching, 21(8), 769-778. https://doi.org/10.1002/tea.3660210802 
Duncan, G. J., Dowsett, C. J., Claessens, A., Magnuson, K., Huston, A. C., Klebanov, P., Pagani, L. S., Feinstein, L., Engel, M., Brooks-Gunn, J., Sexton, H., Duckworth, K., Japel, C., Ginsburg, H., Grissmer, D., Lipsey, M., Neal, D., Raver, C., Sameroff, A., ... Zill, N. (2007). School Readiness and Later Achievement. Psychological Association, 43(6), 1428-1446. https://doi.org/10.1037/[0012-1649.43.6.1428].supp

Fernández-Méndez, L. M., Contreras, M. J., Mammarella, I. C., Feraco, T., \& Meneghetti, C. (2020). Mathematical achievement: the role of spatial and motor skills in 6-8 year-old children. PeerJ, 8. https://doi.org/10.7717/PEERJ.10095

Fisher, K., Hirsh-Pasek, K., Golinkoff, R. M., Singer, D. G., \& Berk, L. (2012). Playing Around in School: Implications for Learning and Educational Policy. In The Oxford Handbook of the Development of Play. https://doi.org/10.1093/oxfordhb/9780195393002.013.0025

Furner, J. M., \& Worrell, N. L. (2017). The Importance of Using Manipulatives in Teaching Math Today. Transformations - The Journal of Inclusive Scholarship and Pedagogy, 3(1), 2. https://nsuworks.nova.edu/transformations/vol3/iss1/2

Glenberg, A. M. (2008). Embodiment for Education. In Handbook of Cognitive Science (pp. 355-372). Elsevier Ltd. https://doi.org/10.1016/B978-0-08-046616-3.00018-9

Glenberg, A. M., Gutierrez, T., Levin, J. R., Japuntich, S., \& Kaschak, M. P. (2004). Activity and imagined activity can enhance young children's reading comprehension. Journal of Educational Psychology, 96(3), 424-436. https://doi.org/10.1037/0022-0663.96.3.424

Grossman, J., \& Mackenzie, F. J. (2005). The Randomized Controlled Trial: gold standard, or merely standard? Perspectives in Biology and Medicine, 48(4), 516-534. https://doi.org/10.1353/PBM.2005.0092

Gunderson, E. A., Ramirez, G., Beilock, S. L., \& Levine, S. C. (2012). The Relation Between Spatial Skill and Early Number Knowledge: The Role of the Linear Number Line. https://doi.org/10.1037/a0027433

Hariton, E., \& Locascio, J. J. (2018). Randomised controlled trials - the gold standard for effectiveness research. BJOG: An International Journal of Obstetrics and Gynaecology, 125(13), 1716. https://doi.org/10.1111/1471-0528.15199

Hirsh-Pasek, K., Michnick Golinkoff, R., Berk, L. E., \& Singer, D. (2010). A Mandate for Playful Learning in Preschool: Presenting the Evidence. In A Mandate for Playful Learning in Preschool: Presenting the Evidence. https://doi.org/10.1093/acprof:oso/9780195382716.001.0001

Holmes, A. B. (2013). Effects of Manipulative Use on PK-12 Mathematics Achievement: A MetaAnalysis. Society for Research on Educational Effectiveness. https://files.eric.ed.gov/fulltext/ED563072.pdf

Hull, D. M., Hinerman, K. M., Ferguson, S. L., Chen, Q., \& Näslund-Hadley, E. I. (2018). Teacher-Led Math Inquiry: A Cluster Randomized Trial in Belize. Educational Evaluation and Policy Analysis, 40(3), 336-358. https://doi.org/10.3102/0162373718768430

Jarrett, O. S. (1998). Playfulness: A Motivator in Elementary Science Teacher Preparation. School Science and Mathematics, 98(4), 181-187. https://doi.org/10.1111/j.19498594.1998.tb17414.x

Jirout, J. J., \& Newcombe, N. S. (2015). Building Blocks for Developing Spatial Skills: Evidence From a Large, Representative U.S. Sample. Psychological Science, 26(3), 302-310. https://doi.org/10.1177/0956797614563338 
Khine, M. S. (2016). Visual-spatial ability in STEM education: Transforming research into practice. In Visual-spatial Ability in STEM Education: Transforming Research into Practice. Springer International Publishing. https://doi.org/10.1007/978-3-319-44385-0

Kirschner, P. A., Sweller, J., \& Clark, R. E. (2006). Why minimal guidance during instruction does not work: An analysis of the failure of constructivist, discovery, problem-based, experiential, and inquiry-based teaching. Educational Psychologist, 41(2), 75-86. https://doi.org/10.1207/s15326985ep4102_1

Kyttälä, M., Aunio, P., Lehto, J. E., Van Luit, J., \& Hautamäki, J. (2003). Visuospatial working memory and early numeracy. Educational and Child Psychology.

Kyttälä, M., \& Lehto, J. E. (2008). Some factors underlying mathematical performance: The role of visuospatial working memory and non-verbal intelligence. European Journal of Psychology of Education, 23(1), 77-94. https://doi.org/10.1007/BF03173141

Lafay, A., Osana, H. P., \& Valat, M. (2019). Effects of Interventions with Manipulatives on Immediate Learning, Maintenance, and Transfer in Children with Mathematics Learning Disabilities: A Systematic Review. Education Research International, 2019. https://doi.org/10.1155/2019/2142948

Levac, D., Colquhoun, H., \& Brien, K. K. O. (2010). Scoping studies : advancing the methodology. 1-9.

Levine, S. C., Ratliff, K. R., Huttenlocher, J., \& Cannon, J. (2012). Early puzzle play: A predictor of preschoolers' spatial transformation skill. Developmental Psychology, 48(2), 530-542. https://doi.org/10.1037/a0025913

Lilienfeld, S. O., McKay, D., \& Hollon, S. D. (2018). Why randomised controlled trials of psychological treatments are still essential. The Lancet Psychiatry, 5(7), 536-538. https://doi.org/10.1016/S2215-0366(18)30045-2

Mayer, R. E. (2004). Should There Be a Three-Strikes Rule against Pure Discovery Learning? The Case for Guided Methods of Instruction. In American Psychologist (Vol. 59, Issue 1, pp. 14-19). https://doi.org/10.1037/0003-066X.59.1.14

Mix, K. S., \& Cheng, Y. L. (2012). The Relation Between Space and Math. Developmental and Educational Implications. In Advances in Child Development and Behavior (Vol. 42, pp. 197-243). Academic Press Inc. https://doi.org/10.1016/B978-0-12-394388-0.00006-X

Mix, K. S., Levine, S. C., Cheng, Y. L., Young, C., Hambrick, D. Z., Ping, R., \& Konstantopoulos, S. (2016). Separate but correlated: The latent structure of space and mathematics across development. Journal of Experimental Psychology: General, 145(9), 1206-1227. https://doi.org/10.1037/xge0000182

Mix, K. S., Smith, L. B., Stockton, J. D. S., Cheng, Y. L., \& Barterian, J. A. (2017). Grounding the Symbols for Place Value: Evidence From Training and Long-Term Exposure to Base-10 Models. Journal of Cognition and Development, 18(1), 129-151. https://doi.org/10.1080/15248372.2016.1180296

Moch, P. L. (2001). Manipulatives work! Educational Forum, 66(1), 81-87. https://doi.org/10.1080/00131720108984802

Moher, D., Liberati, A., Tetzlaff, J., Altman, D. G., Altman, D., Antes, G., Atkins, D., Barbour, V., Barrowman, N., Berlin, J. A., Clark, J., Clarke, M., Cook, D., D’Amico, R., Deeks, J. J., Devereaux, P. J., Dickersin, K., Egger, M., Ernst, E., ... Tugwell, P. (2009). Preferred reporting items for systematic reviews and meta-analyses: The PRISMA statement. In Annals of Internal Medicine (Vol. 151, Issue 4, pp. 264-269). American College of Physicians. https://doi.org/10.7326/0003-4819- 
151-4-200908180-00135

Moher, D., Shamseer, L., Clarke, M., Ghersi, D., Liberati, A., Petticrew, M., Shekelle, P., \& Stewart, L. A. (2015). Preferred reporting items for systematic review and meta-analysis protocols (PRISMAP) 2015 statement. Systematic Reviews, 4(1), 1. https://doi.org/10.1186/2046-4053-4-1

Möhring, W., \& Frick, A. (2013). Touching up mental rotation: Effects of manual experience on 6month-old infants' mental object rotation. Child Development, 84(5), 1554-1565. https://doi.org/10.1111/cdev.12065

Moyer-Packenham, P. S., \& Westenskow, A. (2013). Effects of virtual manipulatives on student achievement and mathematics learning. International Journal of Virtual and Personal Learning Environments, 4(3), 35-50. https://doi.org/10.4018/jvple.2013070103

Moyer, P. S. (2001). Are we having fun yet? How teachers use manipulatives to teach mathematics. $\begin{array}{llll}\text { Educational Studies in } & \text { Mathematics, } & \text { 47(2), }\end{array}$ https://doi.org/10.1023/A:1014596316942

Moyer, P. S., \& Jones, M. G. (2004). Controlling Choice: Teachers, Students, and Manipulatives in Mathematics Classrooms. School Science and Mathematics, 104(1), 16-31. https://doi.org/10.1111/j.1949-8594.2004.tb17978.x

Newman, S. D., Loughery, E., Ecklund, A., You, C., Von Werder, H., \& Soylu, F. (2021). Structured versus free block play: the impact on arithmetic processing. Trends in Neuroscience and Education, 22(November 2020), 100146. https://doi.org/10.1016/j.tine.2020.100146

Pellegrini, A. D., \& Gustafson, K. (2005). Boys' and Girls' Uses of Objects for Exploration, Play, and Tools in Early Childhood. In A. D. Pellegrini \& P. K. Smith (Eds.), The Nature of Play: Great apes and humans (pp. 113-135). Guilford Press. https://doi.org/10.4324/9781351216869-7

Peters, M. D. J., Godfrey, C. M., Khalil, H., McInerney, P., Parker, D., \& Soares, C. B. (2015). Guidance for conducting systematic scoping reviews. International Journal of Evidence-Based Healthcare, 13(3), 141-146. https://doi.org/10.1097/XEB.0000000000000050

Peters, M. D. J., Godfrey, C. M., Mcinerney, P., Baldini Soares, C., Khalil, H., \& Parker, D. (2017). Chapter 11: Scoping reviews. In E. Aromataris \& Z. Munn (Eds.), Joanna Briggs Institute Reviewer's Manual (pp. 141-146). The Joanna Briggs Institute. https://www.researchgate.net/publication/319713049_2017_Guidance_for_the_Conduct_of_J BI_Scoping_Reviews?enrichId=rgreq-2c63bf47a03bf1c379fed09bf9a175b4XXX\&enrichSource=Y292ZXJQYWdlOzMxOTcxMzA00TtBUzo1NDA5MDcxMjY40DY0MDBAMT UwNTk3MzcxNjg4MA\%3D\%3D\&el=1_x_2\&

Pirrone, C., Tienken, C. H., Pagano, T., \& Di Nuovo, S. (2018). The Influence of Building Block Play on Mathematics Achievement and Logical and Divergent Thinking in Italian Primary School Mathematics Classes. Educational Forum, 82(1), 40-58. https://doi.org/10.1080/00131725.2018.1379581

Pound, L. (2006). Supporting mathematical development in early years (O. U. Press (ed.); 2nd ed.). https://books.google.co.uk/books?redir_esc=y\&id=FCFEBgAAQBAJ\&q=shape\#v=snippet\&q=s hape $\& \mathrm{f}=$ false

Pyle, A., \& Danniels, E. (2017). A Continuum of Play-Based Learning: The Role of the Teacher in PlayBased Pedagogy and the Fear of Hijacking Play. Early Education and Development, 28(3), 274289. https://doi.org/10.1080/10409289.2016.1220771

Pyle, A., DeLuca, C., \& Danniels, E. (2017). A scoping review of research on play-based pedagogies in kindergarten education. Review of Education, 5(3), 311-351. 
https://doi.org/10.1002/rev3.3097

Ritchie, S. J., \& Bates, T. C. (2013). Enduring Links From Childhood Mathematics and Reading Achievement to Adult Socioeconomic Status. Psychological Science, 24(7), 1301-1308. https://doi.org/10.1177/0956797612466268

Rittle-Johnson, B., \& Koedinger, K. R. (2005). Designing knowledge scaffolds to support mathematical problem solving. In Cognition and Instruction (Vol. 23, Issue 3). https://doi.org/10.1207/s1532690xci2303_1

Rizzolatti, G., \& Craighero, L. (2004). The mirror-neuron system. In Annual Review of Neuroscience (Vol. 27, pp. 169-192). Annual Reviews. https://doi.org/10.1146/annurev.neuro.27.070203.144230

Sarama, J., \& Clements, D. H. (2009). "Concrete" computer manipulatives in mathematics education. Child Development Perspectives, 3(3), 145-150. https://doi.org/10.1111/j.17508606.2009.00095.x

Simoncini, K., Forndran, A., Manson, E., Sawi, J., Philip, M., \& Kokinai, C. (2020). The Impact of Block Play on Children's Early Mathematics Skills in Rural Papua New Guinea. International Journal of Early Childhood, 52(1), 77-93. https://doi.org/10.1007/s13158-020-00261-9

Skene, K., O’Farrelly, C., Byrne, E. M., Kirby, N., Stevens, E., \& Ramchandani, P. G. (2021). Can Guidance During Play Enhance Children's Learning and Development in Educational Contexts? A Systematic Review and Meta-Analysis. Child Development, (In press).

Sowell, E. J. (1989). Effects of Manipulative Materials in Mathematics Instruction. Journal for Research in Mathematics Education, 20(5), 498. https://doi.org/10.2307/749423

Taylor, M. E., \& Boyer, W. (2020). Play-Based Learning: Evidence-Based Research to Improve Children's Learning Experiences in the Kindergarten Classroom. Early Childhood Education Journal, 48(2), 127-133. https://doi.org/10.1007/s10643-019-00989-7

Thomas, J., Brunton, J., \& Graziosi, S. (2010). EPPI-Reviewer 4.0: software for research synthesis. EPPICentre Software. London: Social Science Research Unit, Institute of Education, University of London.

Thompson, J. M., Nuerk, H. C., Moeller, K., \& Cohen Kadosh, R. (2013). The link between mental rotation ability and basic numerical representations. Acta Psychologica. https://doi.org/10.1016/j.actpsy.2013.05.009

Tolar, T. D., Lederberg, A. R., \& Fletcher, J. M. (2009). A structural model of algebra achievement: Computational fluency and spatial visualisation as mediators of the effect of working memory on algebra achievement. Educational Psychology, 29(2), 239-266. https://doi.org/10.1080/01443410802708903

Toub, T. S., Hassinger-Das, B., Nesbitt, K. T., Ilgaz, H., Weisberg, D. S., Hirsh-Pasek, K., Golinkoff, R. M., Nicolopoulou, A., \& Dickinson, D. K. (2018). The language of play: Developing preschool vocabulary through play following shared book-reading. Early Childhood Research Quarterly, 45(August), 1-17. https://doi.org/10.1016/j.ecresq.2018.01.010

Tran, C., Smith, B., \& Buschkuehl, M. (2017). Support of mathematical thinking through embodied cognition: Nondigital and digital approaches. Cognitive Research: Principles and Implications, 2(1). https://doi.org/10.1186/s41235-017-0053-8

Tricco, A. C., Lillie, E., Zarin, W., O’Brien, K. K., Colquhoun, H. L., Levac, D., Moher, D., Peters, M. D. J., Horsley, T., Weeks, L., Hempel, S., Akl, E. A., Chang, C., McGowan, J., Stewart, L., Hartling, L., 
Aldcroft, A., Wilson, M. G., Garritty, C., ... Straus, S. E. (2018). PRISMA extension for scoping reviews (PRISMA-ScR): Checklist and explanation. In Annals of Internal Medicine (Vol. 169, Issue 7, pp. 467-473). American College of Physicians. https://doi.org/10.7326/M18-0850

Trivette, C. M., Simkus, A., Dunst, C. J., \& Hamby, D. W. (2012). Repeated Book Reading and Preschoolers' Early Literacy Development. Centre for Early Literacy Learning, 5(5), 1-13.

UNICEF. (2015). The investment case for education and equity. In UNICEF Report. www.unicef.org/publications

UNICEF. (2019). A World to Learn: Prioritizing quality early childhood. Global report. https://www.unicef.org/media/57926/file/Aworld-ready-to-learn-advocacy-brief-2019.pdf

Uttal, D. H., Meadow, N. G., Tipton, E., Hand, L. L., Alden, A. R., Warren, C., \& Newcombe, N. S. (2013). The malleability of spatial skills: A meta-analysis of training studies. Psychological Bulletin. https://doi.org/10.1037/a0028446

van Schijndel, T. J. P., Singer, E., van der Maas, H. L. J., \& Raijmakers, M. E. J. (2010). A sciencing programme and young children's exploratory play in the sandpit. European Journal of Developmental Psychology, 7(5), 603-617. https://doi.org/10.1080/17405620903412344

Vander Heyden, K. M., Huizinga, M., \& Jolles, J. (2017). Effects of a classroom intervention with spatial play materials on children's object and viewer transformation abilities. Developmental Psychology, 53(2), 290-305. https://doi.org/10.1037/dev0000224

Verdine, B. N., Golinkoff, R. M., Hirsh-Pasek, K., \& Newcombe, N. S. (2014). Finding the missing piece: Blocks, puzzles, and shapes fuel school readiness. In Trends in Neuroscience and Education (Vol. 3, Issue 1, pp. 7-13). Elsevier GmbH. https://doi.org/10.1016/j.tine.2014.02.005

Verdine, B. N., Golinkoff, R. M., Hirsh-Pasek, K., Newcombe, N. S., Filipowicz, A. T., \& Chang, A. (2014). Deconstructing Building Blocks: Preschoolers' Spatial Assembly Performance Relates to Early Mathematical Skills. Child Development, 85(3), 1062-1076. https://doi.org/10.1111/cdev.12165

Wai, J., Lubinski, D., \& Benbow, C. P. (2009). Spatial Ability for STEM Domains: Aligning Over 50 Years of Cumulative Psychological Knowledge Solidifies Its Importance. https://doi.org/10.1037/a0016127

Weisberg, D. S., Hirsh-Pasek, K., \& Golinkoff, R. M. (2013). Guided play: Where curricular goals meet a playful pedagogy. Mind, Brain, and Education, 7(2), 104-112. https://doi.org/10.1111/mbe.12015

Weisberg, D. S., Hirsh-Pasek, K., Golinkoff, R. M., Kittredge, A. K., \& Klahr, D. (2016). Guided Play: Principles and Practices. Current Directions in Psychological Science, 25(3), 177-182. https://doi.org/10.1177/0963721416645512

Whitebread, D. (2012). Developmental psychology and early childhood education: a guide for students and practitioners. SAGE Publications Ltd.

Whitebread, D. (2019). Overview: The Contribution of Research in Developmental Psychology To Early Childhood Education. In D. Whitebread, V. Grau, K. Kumpulainen, M. M. McClelland, N. E. Perry, \& D. Pino-Pasternak (Eds.), The SAGE Handbook of Developmental Psychology and Early Childhood Education (pp. 1-20). SAGE Publications Ltd. https://doi.org/http://dx.doi.org/10.4135/9781526470393

Wolfgang, C. H., Stannard, L. L., \& Jones, I. (2001). Block play performance among preschoolers as a predictor of later school achievement in mathematics. Journal of Research in Childhood 
Education, 15(2), 173-180. https://doi.org/10.1080/02568540109594958

Wolfgang, C. H., Stannard, L. L., \& Jones, I. (2003). Advanced constructional play with legos among preschoolers as a predictor of later school achievement in mathematics. Early Child Development and Care, 173(5), 467-475. https://doi.org/10.1080/0300443032000088212

Yu, Y., Shafto, P., Bonawitz, E., Yang, S. C. H., Golinkoff, R. M., Corriveau, K. H., Hirsh-Pasek, K., \& Xu, F. (2018). The theoretical and methodological opportunities afforded by guided play with young children. Frontiers in Psychology, 9(JUL). https://doi.org/10.3389/fpsyg.2018.01152

Zosh, J. M., Hirsh-Pasek, K., Hopkins, E. J., Jensen, H., Liu, C., Neale, D., Solis, S. L., \& Whitebread, D. (2018). Accessing the inaccessible: Redefining play as a spectrum. In Frontiers in Psychology (Vol. 9, Issue AUG). Frontiers Media S.A. https://doi.org/10.3389/fpsyg.2018.01124

Zosh, J. M., Hopkins, E. J., Jensen, H., Liu, C., Neale, D., Hirsh-Pasek, K., Solis, S. L., \& Whitebread, D. (2017). Learning Through Play: a Review of the Evidence (White Paper) (Issue November). 


\section{Section 7. Supplementals}

\subsection{Appendix A. Search terms and syntax}

The same key terms were entered into each electronic database search and corresponded to three categories: (1) population/context (child, preschool, elementary, primary school, pre-primary, kindergarten), (2) concept A (manipulative, hands-on material, concrete material, block play, block building, LEGO, DUPLO, origami, paper folding, puzzle, board game, card game, geometric shape, TouchMath, block construction, (play and toy)), and (3) concept B (intervention, curriculum, program, trial, training (experiment and group)). The syntax varied slightly by the database being searched. This is an illustrative example of the search syntax and procedure used in the Child Development and Adolescent Studies database:

Main search:

\section{S1 AND S2 AND S3}

Where:

$\boldsymbol{S 1}=$ TI ( child* OR preschool* OR elementary OR "primary school" OR "preprimary" OR kindergarten) OR AB (child* OR preschool* OR elementary OR "primary school" OR "pre-primary" OR kindergarten) OR KA (child* OR preschool* OR elementary OR "primary school" OR "pre-primary" OR kindergarten )

$\boldsymbol{S 2}=T I$ ( manipulative* OR "hands-on material*" OR "concrete material*" OR "block play" OR "block building" OR LEGO OR DUPLO OR origami OR "paper folding" OR puzzle* OR "board game*" OR "card game*" OR "geometric shape*" OR TouchMath OR "block construction" OR (play* AND toy*) ) OR AB ( manipulative* $O R$ "hands-on material*" OR "concrete material*" OR "block play" OR "block building" OR LEGO OR DUPLO OR origami OR "paper folding" OR puzzle* OR "board game*" OR "card game*" OR "geometric shape*" OR TouchMath OR "block construction" OR (play* AND toy*) ) OR KA ( manipulative* $O R$ "hands-on material*" OR "concrete material*" OR "block play" OR "block building" OR LEGO OR DUPLO OR origami OR "paper folding" OR puzzle* OR "board game*" OR "card game*" OR "geometric shape*" OR TouchMath OR "block construction" OR (play* AND toy*))

$S 3=T I$ (intervention* OR curriculum* OR program* OR trial* OR training OR (experiment* $A N D$ group $\left.{ }^{*}\right) O R$ (experiment* $A N D$ condition*) ) $O R A B$ ( intervention* OR curriculum* OR program* OR trial* OR training OR (experiment* AND group*) OR (experiment* AND condition*) ) OR KA ( intervention* OR curriculum* OR program* OR trial* OR training OR (experiment* AND group*) OR (experiment* AND condition*)) 


\subsection{Appendix B: Screening codes and guidance}

Table B1. Summary of inclusion and exclusion criteria used to guide title/abstract screening and full text screening.

\begin{tabular}{lll} 
Code & \multicolumn{1}{l}{ Requirement for inclusion } & Additional information \\
Codes used for both & the title/abstract and the full-text screening stages \\
\hline Publication date & $2000-2020$ & \\
\hline Language & English & Based on mean age, if available. \\
\hline Sample age & $0-12$ years & $\begin{array}{l}\text { Studies evaluating an intervention or programme targeted } \\
\text { at children (i.e., testing whether the intervention it is } \\
\text { changing something/impacting an outcome; purely } \\
\text { correlation studies are excluded). }\end{array}$ \\
\hline $\begin{array}{l}\text { Empirical study evaluating type } \\
\text { the impact of an } \\
\text { intervention }\end{array}$ & $\begin{array}{l}\text { At least one study group of children that used PMs } \\
\text { (physical, concrete, tangible materials, objects, items) in a } \\
\text { hands-on learning activity (in which they touched, held, } \\
\text { moved, or otherwise manipulated PMs). }\end{array}$ \\
\hline
\end{tabular}

At least one clear and Relevant outcomes related to learning, education, and/or measurable child outcome development, excluding medical measures (e.g., painrelated).

Additional codes used at full-text screening stage only

Excluded if full-text not available after contacting (or attempting contact with) the author(s) or relevant

Full text $\quad$ Full text available institution. Reports were also excluded if the intervention study was not described in full (e.g., an article summary without formal presentation of methods or results).

Sample size $\quad \geq 20 \quad$ Based on total child sample.

Exclude if manipulative is not a focus of the study (e.g., incidental toy play as an element of the study but physical nature of task not important). Additionally, while games used for learning domains such as math and language are

Focus of study $\quad$ PMs for learning included, games used to support acquisition of highly specific subject matter are excluded (e.g., 'education entertainment' such as board and card games to help children learn specific behaviours like handwashing or healthy eating, or that teach a specific information, e.g., about an infectious disease, gangs).

Study included if data are primarily quantitative in nature (e.g., derived from questionnaires, psychological, or educational assessments, observational sampling), studies

Data Quantitative reported exclusively qualitative results are excluded. For reports that presented overlapping outcome data from the same sample, the more recent report was included only (e.g., a $\mathrm{PhD}$ thesis is excluded if the data are reported in a later journal publication).

For inclusion, intervention children physically interacted with PMs. Pencils, pens, and art supplies are not considered Materials Concrete materials/objects PMs in the review. Exclusions apply, including studies with exclusively digital materials (computer-, app- tablet-, smartphone-based), electrical materials (e.g., robotics, 
programmable LEGO/blocks, electronic toys, tangible user interfaces [TUIs]; i.e., tangible objects used to interact with a digital/virtual activity/workspace).

Other (exclusions)

- Studies with PMs used in a hospital setting (e.g., for reducing anxiety/stress) are excluded.

- Therapeutic studies in which the focus of study is LEGO therapy for autism, video modelling (e.g., observing others using materials by video), or studies in which children are engaging with a robot mediator. 


\subsection{Appendix C: Data extraction codes}

Table C1. Data extraction form.

\begin{tabular}{|c|c|c|}
\hline Coding category & Parent codes & Child codes \\
\hline \multirow{5}{*}{$\begin{array}{l}\text { Report } \\
\text { characteristics }\end{array}$} & Year & [Report publication year] \\
\hline & \multirow{4}{*}{ Report type } & Journal article \\
\hline & & $\mathrm{PhD}$ thesis \\
\hline & & Conference report \\
\hline & & Book chapter \\
\hline \multirow{10}{*}{ Sample description } & Sample $N$ & [Report total $N$ in analysis] \\
\hline & \multirow{2}{*}{ Child development } & Typical \\
\hline & & Atypical \\
\hline & \multirow{3}{*}{ Gender } & Male \\
\hline & & Female \\
\hline & & Both \\
\hline & \multirow{4}{*}{ Sample age } & $0-3$ \\
\hline & & $4-6$ \\
\hline & & 7-9 \\
\hline & & $10-12$ \\
\hline \multirow{9}{*}{ Study context } & Country & [Report] \\
\hline & \multirow{4}{*}{ Gross national income } & Low \\
\hline & & Lower-middle \\
\hline & & Upper-middle \\
\hline & & High \\
\hline & \multirow{4}{*}{ Setting } & School/classroom \\
\hline & & Home \\
\hline & & Childcare setting \\
\hline & & Other/unclear \\
\hline \multirow{7}{*}{ Study design } & \multirow{4}{*}{ Research design } & RCT \\
\hline & & Quasi-experimental \\
\hline & & One group (no control) \\
\hline & & Other/unclear \\
\hline & \multirow{3}{*}{$\begin{array}{l}\text { Outcome measure } \\
\text { timepoints }\end{array}$} & Pre- \& post-test \\
\hline & & Post-only \\
\hline & & During-only \\
\hline $\begin{array}{l}\text { Comparison } \\
\text { group(s) (if any) }\end{array}$ & Content & $\begin{array}{l}\text { [Provide brief overview of } \\
\text { activity/activities] }\end{array}$ \\
\hline
\end{tabular}

Note. Data were extracted using, and recoded in, EPPI-Reviewer software. RCT = randomised controlled trial. 
Table C1. (continued). Data extraction form.

\begin{tabular}{|c|c|c|}
\hline Coding category & Parent codes & Child codes \\
\hline \multirow{13}{*}{$\begin{array}{l}\text { Description of } \\
\text { intervention }\end{array}$} & \multirow{6}{*}{$N$ sessions (exposures) } & 1 \\
\hline & & $2-5$ \\
\hline & & $6-10$ \\
\hline & & $11-20$ \\
\hline & & $>20$ \\
\hline & & Unclear \\
\hline & \multirow{4}{*}{ Adult involved } & Teacher \\
\hline & & Researcher \\
\hline & & Parent/caregiver \\
\hline & & Other/unclear \\
\hline & Delivery & $\begin{array}{l}\text { Individual } \\
\text { Group } \\
\text { Classroom } \\
\text { Unclear }\end{array}$ \\
\hline & $\begin{array}{l}\text { Activity/activities } \\
\text { involving PMs }\end{array}$ & [Provide brief overview] \\
\hline & Materials & $\begin{array}{l}\text { Building blocks } \\
\text { LEGO/DUPLO bricks } \\
\text { LEGO engineering } \\
\text { Figurines \& other small toys } \\
\text { Math PMs } \\
\text { Geometric shapes } \\
\text { Paper/card (for folding) } \\
\text { Construction sticks } \\
\text { Science-based materials } \\
\text { Puzzles } \\
\text { Other/unclear }\end{array}$ \\
\hline $\begin{array}{l}\text { Outcomes \& } \\
\text { main findings }\end{array}$ & $\begin{array}{l}\text { Math/numeracy } \\
\text { Literacy/language } \\
\text { Spatial/visual-spatial } \\
\text { Science-related } \\
\text { Social-emotional } \\
\text { Visual perception } \\
\text { Play-based } \\
\text { Fluid intelligence } \\
\text { Executive function } \\
\text { Memory } \\
\text { Other }\end{array}$ & $\begin{array}{l}\text { [Describe outcome measures \& } \\
\text { main findings] }\end{array}$ \\
\hline
\end{tabular}

Note. Data were extracted using, and recoded in, EPPI-Reviewer software. 


\subsection{Appendix D: Main outcome domains and findings by study grouping}

\subsubsection{Block building}

Borriello (2018) - Older children placed more blocks during both LEGO free play and LEGO construction, reflecting better overall block building skills with increasing age. Spatial language: Child spatial language during two LEGO play activities was higher for the intervention vs. control group (ANOVA; main effect of group). Play-based: Intervention children engaged in less pretend play in the LEGO free-play task than controls (ANOVA; main effect of group).

Boyle (2017) - Visual-spatial: Improvements in spatial reasoning (as measured by a spatial transformation task; ANCOVA; main effect of time). Note. This study lacks a comparison group.

Brey (2017) - Visual (perception): The Six Bricks group improved on nine subtests of a Visual Perceptual Aspects Test, whereas no-intervention controls only showed gains in three (statistical models unclear; no group comparisons made). Fluid intelligence: Both groups showed gains in reasoning ability (Raven's Matrices), but no group comparisons were made (statistical model unclear).

Bugos (2017) - Executive function: Overall, children improved on an inhibition task (Day/Night Stroop), but there were no group differences (ANOVA; main effect of time but not group, and no interaction). On another inhibition measure (Matching Familiar Figures), performance was worse after LEGO vs. music training (ANOVA; disordinal group X time interaction).

Casey (2008a) - Visual-spatial: Three tasks, each assessed using MANCOVA (pre-test as covariate; post-hoc LSD if main effects of group). Following the interventions, children in the two block building groups (with or without the story) outperformed controls on a spatial reasoning measure (Block Design subtest of WISC-IV). The block play with story group also had better scores than the other two groups on a block building task. There were no group differences on a mental rotation ask.

Christakis (2007) - Spatial language: Language acquisition scores (measured via the MacArthurBates Communicative Development Inventories) were higher for middle- and low-income children who received blocks vs. controls (effect not found in sample as a whole; multivariate regression). Executive function: No differences in attention (hyperactivity subdomain of Child Behaviour Checklist; multivariate regression). Note. No pre-test data was collected in this study.

Ferrara (2011) - Spatial language: Children in the guided-play condition engaged in more spatial talk than those in the free-play group, and there were no differences between the pre-assembled and free-play groups, or between the guided-play and pre-assembled groups (ANOVA, main effect of group; followed-up with $t$-test comparisons).

Goldstein (2018) - Social-emotional: Various measures were assessed (including theory of mind, altruism, comforting, helping, emotion matching, and social interaction). The control groups in the study (block building and story time) were collapsed and so the first author was contacted for details regarding the block building group compared to the other conditions. There were no group differences between block building and the other groups on any measure.

Harn (2019) - Social-emotional: Four Positive Emotion sub-scales (joy, empathy, contentment, selfconfidence), and five Emotional Competency sub-scales (expressive, transformation, management, 
regulation, autonomy) assessed at outcome. No interpretation is made here due to errors in reporting of results in the paper. For example, it appears that $M$ and $S D$ values are transformed for the control group post-test transformation score (see Table 3, pg. 7). Further, the $p$-values associated with $t$ scores at baseline (see Table 2, pg.7) appear inaccurate. For example, the experimental and control $M(S D)$ at baseline $=27.08(3.06)$ and $18.50(4.46)$, respectively $(t=5.50 ; p=.24)$, and at post-test $=$ 27.08 (2.54) and 22.75 (7.25), respectively $(t=1.95, p=.001)$. Despite the significantly higher post-test scores for experimental vs. control, and non-significant pre-test group differences (as reported in the paper), the experimental group mean scores did not change and the control group improved.

Jemutai (2019) - Visual (perception): $t$-tests compared mean pre-to-post difference between groups on a Visual Perception Aspects Test for the South Africa and Kenya samples separately. No difference was reported for the South Africa sample, and larger gains were found for the intervention vs. nointervention group for the Kenya sample. Note. The authors report an adjusted $\alpha(10 \% ; p \leq 0.1)$ due to small sample. However, the change in confidence level and small sample increase the chance of Type I error. There were no effects when using $p<0.05$.

Newman (2016) - Visual-spatial: No group differences in accuracy or RT on a mental rotation task (ANOVAs; no group X time interactions or main effects of group). Despite the lack of interactions the authors conducted separate within-group ANOVAs that indicated gains for the block play but not word boardgame. Other: Brain imaging ( $f M R I)$ showed that block play was related to increased activation in the para-hippocampal gyri, cerebellum, and fusiform gyri (regions implicated in spatial processing).

Pirrone (2018) - Math/numeracy: Greater gains in math for the block building versus control group ( $t$-test; no group differences between pre-test scores; Objective Mathematical Tests for Primary School). Visual-spatial: Greater gains on a mental imagery task for the block play than control group ( $t$-test; no group comparison made at baseline). Fluid intelligence: Larger gains in non-verbal reasoning for the block play versus control group (Raven's Matrices; $t$-test, no group difference at baseline). Other: No difference in logical thinking score changes (Logical Operations and Conservation test; $t$-test). Greater gains on a creative personality test for the block play versus control group ( $t$-test, no difference at pre-test).

Sawyer (2019) - Other: Drawing skills were measured over the course of the intervention and multilevel models (controlling drawing scores at start of intervention) revealed effects of both time and group. Pairwise comparisons indicated that the block building and story time conditions resulted in better overall drawing than a dramatic pretend play condition (but no difference between the block play and story time groups). In terms of drawing sub-dimensions, story time was more beneficial than dramatic pretend play for creativity, talent, and spatial complexity, while block building was more beneficial than dramatic pretend play for children's use of colour.

Schmitt (2018) - Post-test scores of all outcome measures analysed using regression models controlling for pre-test. Math/numeracy: No group differences in early numeracy, shape recognition, or math language. Executive function: No group differences in inhibition (Sun/moon Stroop), cognitive flexibility (Card Sorting), or global EF (Head-Toes-Knees-Shoulders).

Schröder (2019) - General linear mixed models used for all outcome measures (group X time). Executive function: No impact on attention. Visual (perception): The book reading group got 
slightly worse, whereas the block play group improved, on a test of visual form sensitivity (disordinal group X time interaction). Pairwise comparisons ( $t$-tests) revealed gains for the block play but not book reading group. However, there were no effects on numerosity discrimination.

Simoncini (2020) - Math/numeracy: Overall, the intervention children who received blocks outperformed controls who did not receive blocks ( $t$-test), as assessed via the Schedule for Early Number Assessment (a task-based interview used to elicit strategy use across five areas: subitising, number identification, forward number sequence, backward number sequence, early arithmetic). $t$ tests showed higher scores for the intervention versus control group on backward number sequence and early arithmetic strategies, but no differences were found on the remaining sub-tests). Note. Baseline data was not collected.

Tian (2021) - Note that while a total of 65 children completed the study (presence of post-test data; 70 recruited), the authors based their main analyses on a subset of 46 children (a sub-sample with 23 children in each group who were matched on several variables, matching on age, gender, parental education, family income, IQ, and in baseline scores of constructive play frequency, block building ability, inhibition, spatial ability, visual perspective taking [VPT], and theory of mind [ToM]). Visualspatial: Children in the block play group showed greater gains in spatial ability than controls (indicated by a group X time interaction, ANOVA, and pairwise comparisons showed pre-to-post gains for the block play but not drawing group). Pairwise comparisons showed pre-to-post gains in VPT in the block play but not control group, however there was no group X time interaction (ANOVA) suggesting VPT was not differentially impacted by group. Social-emotional: A group X time interaction (ANOVA) and pairwise comparisons revealed pre-to-post gains to ToM in the block play but not control group. When co-varying for post-test baseline scores of the block play group (ANCOVA) the benefits to ToM disappeared, suggesting the gains in ToM may have been mediated by VPT.

Vander Heyden (2017) - Visual-spatial: There were no group differences in pre-to-post scores of mental rotation or mental folding (ANOVA). A group X time interaction revealed greater gains in spatial perspective taking for the intervention versus control group (however, ANOVA does not control for group differences at baseline). Intervention progress was not associated with children's socioeconomic status or sex.

Willson-Quayle (2001) - Outcomes analysed with ANOVAs (group X time). Visual-spatial: Children's accuracy replicating a DUPLO model improved overall (main effect of time). While the overall gains were mediated by improvements in the adult-scaffolded and adult-directed conditions (scores decreased for the free-play condition), no group differences were found. Social-emotional: Outcomes coded from video recordings of children during block play. No impact on happiness scores, but an overall increase in negative feelings (and a main effect of time for both sadness and anger/frustration, but no group difference). There was a group $\mathrm{X}$ time interaction for persistence (on-task behaviour and focus): the adult-scaffolded and free-play groups had similarly higher levels at pre-test compared to the adult-directed group. While persistence increased in the adult-scaffolded and adult-directed groups, it decreased in the free-play group (but no pairwise comparisons were reported). No differences were found in private speech scores. 


\subsubsection{Math-based PMs}

Aleid (2015) - Math/numeracy: All groups showed significant pre-to-post gains in a math attainment test, and there were no pre-test differences among groups (separate ANOVAs). Group comparisons (ANOVA) of gain scores revealed a group effect. Post hoc tests (Bonferroni) showed that peer tutoring alone group showed larger gains than the control group, and that the group who received the manipulatives + PT intervention had greater gains than the other three groups.

Alghazo (2010) - Math/numeracy: Counting skills assessed via nine measures (forward counting, backward counting, number after, number before, skip counting, one-one-correspondence principle, cardinality, production of sets, comparing quantitates). No group differences at baseline and significant pre-to-post gains for both groups on all measures ( $t$-tests). The intervention group outperformed the traditional teaching group at post-test on all measures.

Alshehri (2018) - Math/numeracy: Steeper gains for reported for the physical and virtual manipulatives groups relative to no-intervention controls on a fractions test, but there were no differences between the physical and virtual manipulatives groups (ANOVAs).

Bennett (2000) - Math/numeracy: No group differences in performance on a test of early math ability.

Cramer (2002) - Math/numeracy: Pupils taught with the manipulatives curriculum (RNP) outperformed those taught with the textbook on a test of fraction learning/retention (MANOVA). Note. No pre-test data was collected in this study.

Dennis (2012) - Math/numeracy: There were no group differences between scores on a volume and capacity measure at pre-test, but the pupils in the manipulatives group outperformed those in the traditional teaching group at post-test (Mann-Whitney $U$ tests).

Eason (2020) - Literacy/language: Group differences found in math vocabulary scores during the intervention (ANOVA); post hoc comparisons revealed that children in the formal learning (no touching) group produced more math talk than the other two groups (guided play and free play), and that those in the guided play group engaged in more math talk than free play controls.

Ermakova (2017) - Math/numeracy: Children who used two types of materials (base-10 frames and tiles) did not differ from children who only used one type (base-10 frames or tiles) in their likelihood of using a mental base-10 decomposition strategy at post-test (ANCOVA).

Fisher (2011a) ${ }^{34}$ - Math/numeracy: Children were better at shape sorting after completing the either guided play or didactic activities versus those who engaged in free play, but there were no group differences on an embedded shapes task (MANOVAs and follow-up comparisons). Note. No pre-test data was collected in this study.

Fisher (2011b) ${ }^{34}$ - Math/numeracy: Guided-play benefited shape sorting more than didactic instruction or free play, and didactic instruction benefited more than free play. Guided play also benefited performance on an embedded shapes task versus free play, but there was no difference in

${ }^{34}$ Fisher (2011a) and Fisher (2011b) report on different participants, Fisher (2013) reports on the same sample as Fisher (2011b) but with additional participants. 
improvements between guided play and didactic instruction or between free play and didactic instruction (MANOVAs and follow-up pairwise comparisons). Note. No pre-test data was collected in this study.

Fisher (2013)34 - Math/numeracy: The guided-play intervention benefited shape sorting ability more than didactic instruction or free-play (MANOVA and post hoc comparisons).

Fujimura (2001) - Math/numeracy: Results from experiment one indicated that both groups who worked on proportional reasoning problems during the intervention (with or without PMs) showed greater improvements in their ability to solve juice concentration comparison problems than a control group who worked on non-proportional math problems (without PMs; ANOVA: group X time interaction). Post hoc pairwise comparisons also revealed that both the PM and non-PM proportional reasoning groups showed pre-to-post gains, but that the other control group did not. Further, the PM group outperformed the control group at post-test, but no other groups differences were found between post-test scores.

Gecu-Parmaksiz (2018, 2019)35 - Visual-spatial: Larger gains on two measures of spatial ability (the Picture Rotations Task and Spatial Perception Scale) for the AR versus PMs group ( $t$-tests and ANOVAs; no baseline differences; findings reported in Gecu-Parmaksiz, 2018). Math/numeracy: Children in the virtual group demonstrated larger gains on a Geometric Shape recognition task than those in the physical group. Note. Only $t$-tests were used for within- and between-group comparisons, which do not account for baseline variations. The authors report a significant group difference on pre-test scores, which may have mediated observed differences at post-test (findings reported in Gecu-Parmaksiz, 2019).

Hawes (2017) - Math/numeracy: No differences in non-symbolic magnitude comparison or number knowledge. A time X group interaction for symbolic magnitude comparison favoured the PM intervention group (ANOVA, no follow-up comparisons). Receptive \& spatial language: No effects for receptive vocabulary (PPVT). A group X time interaction for spatial language favoured the PM intervention group (but the inquiry-based control group outperformed intervention children at pretest, and the authors report a group difference between post-test scores, despite a $p>.05$, therefore, the interaction should be interpreted with caution). Math-spatial: Group X time interactions (ANOVA) showed greater gains for the PM intervention group than controls in visual-spatial geometry and mental rotation. Post hoc $t$-tests revealed post-test differences for geometry (favouring Group A) but not mental rotation. Note. There were no group differences at baseline for any measure except spatial language ( $t$-tests).

Horan (2018) - Math/numeracy: No group differences in counting.

Hull (2018) - Math/numeracy: Eight grade-appropriate math achievement measures were employed (using items from the Michigan Math Leadership Academy assessment). Results from an intention-to-treat (ITT) MLM with three levels accounting for clustering (pupils, teachers, schools) and pre-test scores revealed a positive intervention effect. An additional test-of-treatment (TOT)

\footnotetext{
35 Gecu-Parmaksiz (2018) and Gecu-Parmaksiz (2019) report on the same study and sample in two journal publications and so have been grouped for the review.
} 
MLM considering the level (fidelity) of implementation also found a positive effect related to increases in teacher implementation.

Martin (2012) - Math/numeracy: Both groups improved but there were no group differences in fraction problem accuracy during the intervention session.

Mattoon (2015) - Math/numeracy: Both groups improved but there were no group differences in early math ability.

Mendiburo (2011) - Math/numeracy: Children in the virtual manipulatives group outperformed those in the PMs group on a fraction assessment at post-test and were able to complete more exercises during the intervention (ANCOVA with pre-test as covariate).

Moyer-Packenham (2013) - Math/numeracy: No differences in fraction learning between the physical and virtual manipulatives groups for third or fourth grade pupils.

Sophian (2004) - Math/numeracy: Pupils in the math-based PM curriculum outperformed those in a literacy-based curriculum and no-intervention controls at post-test on a math ability measure (items relating to shapes, patterns, counting, numerals, sets, ordinal positions, and logical operations) and a measurement and shapes task (ANCOVAs accounting for pre-test scores and follow-up comparisons).

Starkey (2004) - Math/numeracy: Children in the intervention group showed pre-to-post gains in composite scores on the Child Math Assessment (ANOVA). Group comparisons were conducted with ANCOVAs (covarying for age but not pre-test; and no pre-test data collected from comparison group). The intervention had higher post-test scores than the control group. Furthermore, middle-income children receiving the intervention outperformed low-income children at pre- and post-test. Both showed gains, and an interaction revealed that the low-income sample had greater gains than the middle-income children (ANOVA and planned post hoc comparisons), and at post-test the lowerincome intervention children were statistically similar to middle-income who did not receive the intervention.

Taylor (2001) - Math/numeracy: Children in the computer instruction showed larger gains than those who received traditional teaching on a probability measure (ANCOVA controlling for pre-test).

Thompson (2012) - Math-spatial: All intervention groups receiving manipulatives and/or multimedia outperformed children who only received regular instruction on a measure of math (focusing on geometry and spatial visualisation), but no other groups differences among the instructional groups were reported (ANCOVA covarying pre-test and Tukey's HSD follow-up tests).

Verdine (2019) - Literacy/language: Children in who took part in the intervention (standard and alternate shape PMs) produced more words overall, shape names, and spatial talk than those in the other two groups (regression analyses).

Watchorn (2011) - Math/numeracy: All intervention groups involving math equivalence problems (with or without PMs) performed better at outcome (accuracy on equivalence problems) than controls who received instruction on an unrelated math topic, however there was no impact of PMs. 


\subsubsection{Reading/literacy-based PMs}

Biazak (2010) - Memory (verbal): Memory for narrative passages (free- and cued-recall questions related to story events). Floor effects were found at baseline for free-recall (scored for nonaction and action propositions), therefore scores for this outcome were coded as binary (low/high) and entered into separate (action and nonaction) GLMs (controlling for baseline scores). A main effect of group revealed greater scores for the PM intervention than the listening-only control group. Further, this effect was relatively greater for children who were initially better at remembering story content (coded as high pre-test). There was also a main effect of age, but it did not interact with condition. There were no group (or age) effects for nonaction propositions. For the cued-recall outcome, group predicted post-test scores: children in the intervention answered more cued-recall questions correctly than controls. There was no effect of age. There was an additional within-subjects factor of sentence type (typical or atypical) in the statistical model, but the second hypothesis that children in the intervention would show greater memory gains for atypical than typical story events was not supported (based on lack of interaction and descriptive means).

Cavanaugh (2017) - Literacy/language: In this study, all participants completed both the experimental (guided play with PMs) and control (direct instruction with PMs) conditions (both groups completed each condition for three weeks, counterbalanced, over the course of six weeks). Gains scores for the intervention and control groups were calculated at three- and six-week time points (yielding combined post-test scores with the whole sample included in both the intervention and control conditions). A paired $t$-test was then conducted comparing participants' overall gains scores in the experimental versus control intervention. Children demonstrated greater improvements in literacy (Dynamic Indicators of Basic Early Literacy Skills; DIBELS) following the guided play versus adult-directed intervention.

Cobb (2001) - Literacy/language: Analyses ( $t$-tests; unclear if post-test or gain scores were entered as the dependent variable) revealed selective benefits to children's reading scores (Gates-MacGinitie Reading Test), which varied by grade and by sub-test: first graders in the experimental group outperformed controls on a vowels subtest and in total reading scores. There were no differences for three other subtests (initial consonants/clusters, final consonants/clusters, use of sentence context). No group differences were reported of the three sub-tests completed by grades two or three (vocabulary, reading comprehension, reading total). Note that $t$-tests do not control for possible baseline variations.

Dickinson (2019) - Data were analysed with MLMs (accounting for clustering in classrooms). Literacy/language: There were no group differences in receptive or expressive vocabulary scores for target (task-relevant) or control (novel) words. Social-emotional: There were no group differences in self-regulation (peg-tapping) or teacher-rated behavioural regulation (Cooper-Farran Work-Related Skills test) scores.

Han (2010) - Literacy/language: The reading plus play intervention group had larger gains than the reading-only controls in expressive vocabulary scores (Individual Growth and Development Indicators: Picture Naming measure), however there was no group difference in gains for receptive vocabulary scores ( $t$-tests; analyses did not account for potential baseline variations). 
Lane (2009) - Literacy/language: Scores on four reading measures were assessed at outcome (ANCOVAs co-varying baseline scores). Comparisons were made between each intervention group and the control group, but not among the intervention groups. Children in the four intervention groups did no better than passive controls on a decoding task. Children who received the whole programme did better on a sight word test than controls (however no comparison was made between children who received the same programme with or without PMs). Relative to no-intervention controls, all four groups who received the programme (with or without PMs/sentence writing/extending literacy) showed improvements in phonological awareness. However, as no comparisons were made between the intervention groups, it cannot be determined whether there is any additive benefit of PMs to children's phonological awareness. Finally, children in the whole programme, or in the programme without the extended literacy step, did better on a reading task (Word Attack subtest of the Woodcock Diagnostic Reading Battery; note that baseline phonological awareness scores, not word attack scores, served as a covariate). However, as comparisons were not made among the intervention groups it is difficult to draw conclusions about the additive benefits of individual components of the literacy programme.

Toub (2018) - Literacy/language: Scores of receptive and expressive vocabulary tests (using target words from the intervention and novel words) were analysed with MLMs (accounting for nesting in classrooms and controlling for pre-test scores). Results indicated that both guided- and directed-play resulted in benefits to both receptive and expressive vocabulary relative to the free-play condition. There were no differences between the guided and directed play groups.

Weisberg (2015) - Literacy/language: Both groups showed gains in expressive and receptive vocabulary. There were no group differences for receptive vocabulary, but there were larger gains in expressive vocabulary for the fantastical than realistic conditions. Note that the two conditions differed only by story theme (realistic versus fantasy) and the activities were the same (reading plus play with toy props). As there was no additional control group, any observed intervention benefits cannot be attributed to the PM elements of the study. Data were analysed via ANOVAs and $t$-tests.

\subsubsection{Science-based PMs}

Bulunuz (2013) - Science-based: Data collected via interviews yielding seven science-based outcomes (living/non-living, colour, water, gravity, air, magnets, floating/sinking). No baseline differences were found on any (Chi-Square). Group comparisons using Chi-Square (based on postinterview scores) revealed effects favouring the playful hands-on group on all seven science concepts compared to didactic comparison group.

Dejonckheere (2016) - Play-based: Children's exploratory play was assessed $(N$ meaningful/informative actions/experimentation in a light box task). Children in the guided play group showed greater gains than controls (MANOVA, main effect of group and a time X group interaction).

Lazonder (2014) - Science-based: A beliefs questionnaire was used to assess misconceptions about height, mass, and size (but height dropped from analysis due to there being too few misconceptions at pre-test). Chi-square tests were used for all comparisons. There were no baseline differences between groups. At post-test, most children corrected their initial misconceptions about size, but this was independent of condition. Children in the physical dropping intervention were more likely to 
revise their misconceptions about mass than those who simply observed or engaged with virtual materials.

Li (2016) - Science-based: Both groups showed gains on a physics measure, but there were no group differences. Other: Problem solving 'ability' was measured by a self-assessment questionnaire on five dimensions related to the five aspects of the design process (developed by the author; i.e., strategy use), but there were no improvements for either group.

Lu (2018) - Other (learning retention - science-related): Assessed children's learning from the intervention task (appearance, behaviour, footprints, and fossils of dinosaurs). There were no differences between groups at baseline, both groups showed pre-to-post gains, and the post-test score for the virtual manipulatives group was higher than that of the PMs group ( $t$-tests). There were no group differences in motivation scores.

Marulcu (2016) - Science-based: Post-test scores were entered into separate ANCOVAs (covarying for group and pre-test). Children's science content learning of simple machines was assessed via written tests (multiple choice and open-ended questions) and interviews. There were no groups differences in gains for the written test scores, but the LEGO engineering group outperformed the hands-on inquiry-based control group on the interview questions.

Portsmore (2010) - Science-based: Children completed both non-LEGO (craft materials not present in the intervention) and LEGO (same as the intervention materials) engineering design problems at pre- and post-test (e.g., retrieve keys from the bottom of a tall box). The data used for analyses were the drawings (engineering design problem plans) that students made before constructing their solutions, and their final constructed artifacts (Mann-Whitney $U$ tests). Overall (independent of group), students improved in making solutions with intervention (LEGO) but not novel (non-LEGO) materials. However, intervention (with or without planning stage) type did not impact the effects.

van Schijndel (2010) - Play-based: Children's exploratory play behaviours were videotaped at preand post-test and coded using the Exploratory Play Scale. Levels of exploratory play increased in the intervention (guided play in sandpit) but not control (free play) group (group X time interaction; ANOVA). However, controls engaged in more exploratory play than intervention children at baseline, which may have mediated the effects (e.g., reflecting regression to the mean). Therefore, the authors conducted additional group comparisons using a subset of matched participants to ensure balance at baseline $(n=14)$. The intervention group had higher levels of exploratory play than controls at posttest $(t$-test). Initial exploratory play levels were also found to moderate intervention improvements: an interaction between group and initial levels of exploratory play indicated that children with lower levels of exploratory play benefited most from the intervention (ANOVA).

Zacharia (2012) - Note that the pre-test, intervention, and post-test all happened in the same single session. Science-based: Data were collected via a 'clinical interview'. The outcome measure (balance beam understanding) involved children judging and explaining which of two objects was heavier. Children with higher versus lower levels of 'correct prior knowledge' were analysed independently. Both groups of children with correct prior knowledge improved, regardless of manipulatives condition (physical versus virtual). Children with incorrect prior knowledge in the PM group improved (and caught up to the children with correct prior knowledge), but those in the virtual condition did not (findings based on ANOVAs and post hoc $t$-test/Bonferroni comparisons). 


\subsubsection{Paper folding}

Boakes (2009) - Math-spatial: No effects on a geometry measure (ANCOVA). Visual-spatial: Spatial visualisation skills were measured using three subtests of the Kit of Factor-Referenced Cognitive Test and analysed with three separate ANCOVAs: there were no group differences for 3D visualisation ability (paper folding task) or in the ability to move between dimensions (surface development task) at post-test. For the final sub-measure - 2D visualisation ability (card rotation task) - there was no main effect of group, but there was a group X gender interaction (males scored higher than females following origami instruction, and while males in the experimental group outperformed controls, females in the intervention performed worse than controls).

Burte (2017) - There was no comparison group in this study. Results based on linear regression models. Math/numeracy: Overall (grades combined), math accuracy increased marginally from preto post-test (but while by grades 5 and 6 improved, grades 3 and 4 decreased slightly). Visual-spatial skills: Overall (grades combined), there were gains in accuracy for paper folding and in a rotations task (though accuracy at post-test remained quite low for the latter [35\%], and this overall gain was driven by gains made for grade 3 children). Finally, overall performance in a 'make-a-dice' task decreased.

Cakmak (2014) - Visual-spatial: A spatial ability test (SAT) was adapted from a 2D spatial geometry task and a paper folding task; there were pre-to-post gains for spatial visualization scores and spatial orientation scores ( $t$-tests). However, this study lacked a control group.

Krisztián (2015) - Visual-spatial: Pairwise comparisons ( $t$-tests) revealed gains in spatial ability (shape counting, mental rotation, mental knotting) for the experimental but not control groups, however, there was no group X time interaction (ANOVA), despite there being a medium effect. Math/numeracy: there was a binomial group X time interaction for numerical ability (addition, subtraction, multiplication, division); numerically there were gains for the intervention children but not controls, plus a main effect of group but not time. This provides some limited evidence that the intervention had a positive impact relative to controls, however, no follow-up within- or betweengroup comparisons were reported, and effects could be influenced by baseline variations.

Raimundo (2013) - In this study, the origami group were controls, and were compared the main intervention group (a socio-emotional learning program). Social-emotional: There was no benefit of the origami curriculum versus the social-emotional learning program for various social-emotional outcomes (e.g., emotional knowledge, social competence).

Taylor (2013) - Visual-spatial: there were no group differences in gain scores (analysed via $t$-tests) for mental paper folding or mental rotation. The intervention versus control improved more on a 'Make a Die' task, however the analyses did not account for the potential impact of baseline variations.

Yuzawa (2002) - In this study performance was assess during the intervention (rather than at pre/post-test). Math/numeracy: Children in the experimental group (origami and size comparison tasks) improved more than controls (size comparison tasks only) on the size comparison tasks (ANOVA; post hoc comparisons showed an increase from day 3 to 5). The origami intervention also had a positive impact in effective size comparison strategies. 


\subsubsection{Puzzles \& Tangrams}

Casey (2008b) - Math-spatial: Geometry triangles task (a subtest from the Kaufman-Assessment Battery for Children) and a Tangram task (both involved part-whole-puzzle problems). There were group differences at baseline in the triangle, but not Tangrams, task: controls scored higher than those in the intervention. Results for the triangles task revealed a group X time interaction showing greater improvements for the intervention versus control group (ANOVA and ANCOVA covarying pre-test scores). Further subgroup analyses revealed that the interaction was present for girls but not boys. There were no group differences in pre-to-post gains for the Tangram test (ANOVA and ANCOVA controlling for pre-test).

Casey (2008c) - Math-spatial: Same tasks as above. There were no baseline differences between groups on either task. A group X time interaction (ANOVA) revealed larger pre-to-post gains for the geometry plus story group compared to the geometry only control group on the triangles task, but unlike in study 1 (see Casey 2008 b above), there was no impact of sex on these effects. There was a group $\mathrm{X}$ time interaction showing greater gains for the intervention versus control group on the Tangram task (ANOVA), and also an interaction with sex: there were larger gains for girls than boys.

Dang (2018) - Other (learning retention - geography): Learning retention from the intervention puzzle task (marking countries/continents on world and Asia maps): there was no group difference in scores for the Asia map, but the non-puzzle learning was better than the puzzle learning for the world map (Mann-Whitney Analyses of $z$ scores).

Eisen (2020) - Other (learning retention - geography): Recognition and recall of Australian states (composite memory score) was assessed (data for three comparison groups was collected during three separate studies; results here are based on those reported in study three when data were aggregated). Note that findings are based on post-test scores only (no baseline measure). There was a main effect of group (ANOVA) and post hoc (Bonferroni) tests revealed that both the physical and app-based puzzle activities with adult guidance/direction resulted in greater scores than using the app without an adult (the social physical and app-based puzzle groups did not differ).

Olkun (2003) - Math-spatial: Composite geometry score from a test involving various types of questions (spatial visualisation, spatial-numeric, area measurement, mental rotation). There was a main effect of group on gain scores (ANOVA). Post hoc analyses revealed that both the physical and computer-based puzzle groups showed larger improvements relative to the no-intervention controls, but that the gains between physical and computer-based groups did not differ.

Thompson (2016) - Math/numeracy: There were no group differences in baseline math achievement (assessed via a condensed version of the Second Grade California Standards Mathematics Test; data analysed with Kruskal-Wallis test, like ANOVA for rank data). Pre-to-post changes analysed using Wilcoxon Signed Ranks test with Z-test statistics (for rank data): post-test scores were not different to pre-test for any of the three groups and a Kruskal-Wallis Test found no group differences in post-test scores. Visual-spatial: There were no pre-test variations in spatial ability (Children's Mental Transformation Task) among groups (Kruskal-Wallis test). There were no pre-to-post differences for the PM or multimodal groups (Wilcoxon Signed Ranks tests), but there was an improvement for the virtual manipulatives group (moderate effect size). However, there were no group differences in post-test scores among groups (Kruskal-Wallis test). 


\subsection{Appendix E. Other included studies}

\section{Interventions involving basic toy play/exploration $(n=2)$}

Table E1. Characteristics of studies in involving basic toy play/exploration $(n=2)$

\begin{tabular}{|c|c|c|c|c|c|c|c|c|c|c|c|c|}
\hline ID & Location & $\begin{array}{c}\text { Sample } \\
N\end{array}$ & Age & $\begin{array}{c}\text { Research } \\
\text { design }\end{array}$ & Setting & $\begin{array}{l}\text { Model of } \\
\text { delivery }\end{array}$ & $\begin{array}{c}\text { Adult } \\
\text { involved }\end{array}$ & $\begin{array}{c}N \\
\text { sessions } \\
\end{array}$ & Materials & Intervention & Comparison(s) & $\begin{array}{c}\text { Main outcome } \\
\text { domains }\end{array}$ \\
\hline $\begin{array}{c}\text { Clearfield } \\
2019\end{array}$ & USA & 42 & $0-3$ & $\begin{array}{l}\text { RCT, pre- } \\
\text { post }\end{array}$ & Home & Individual & $\begin{array}{l}\text { Parent/ } \\
\text { caregiver }\end{array}$ & $11-20$ & Toy rattle & $\begin{array}{c}\text { Parents demonstrated \& encouraged } \\
\text { two manipulative actions (e.g., poke) } \\
\text { with a rattle in daily play sessions with } \\
\text { infants }\end{array}$ & $\begin{array}{l}\text { 1. Structured direction } \\
\text { with seven actions } \\
\text { 2. Unstructured } \\
\text { direction/free play }\end{array}$ & $\begin{array}{l}\text { Exploratory } \\
\text { play (physical/ } \\
\text { motor skills) }\end{array}$ \\
\hline $\begin{array}{c}\text { Cooper } \\
2014\end{array}$ & $\begin{array}{l}\text { South } \\
\text { Africa }\end{array}$ & 30 & $0-3$ & $\begin{array}{l}\text { RCT, pre- } \\
\text { post }\end{array}$ & Home & Individual & $\begin{array}{l}\text { Parent/ } \\
\text { caregiver }\end{array}$ & $>20$ & $\begin{array}{l}\text { Unclear } \\
\text { (toys) }\end{array}$ & $\begin{array}{l}\text { Toy play: mothers were given a new } \\
\text { toy each week \& engaged their child in } \\
\text { play sessions everyday }{ }^{1}\end{array}$ & Book sharing & $\begin{array}{l}\text { Literacy/ } \\
\text { language, } \\
\text { executive } \\
\text { function }\end{array}$ \\
\hline
\end{tabular}

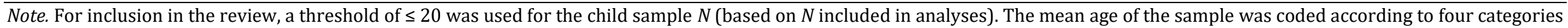

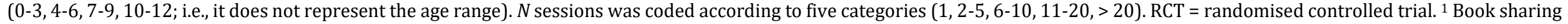
was the main intervention group and toy play was the control group in this study.

\section{Main outcome domains \& findings}

Clearfield (2019) - Exploratory play (physical/motor skills): Infants' physical explorations with a toy were coded at pre- and post-test (e.g., poking, squeezing, rotating); ANOVAs used for data analyses. There were no gains in exploratory behaviours of the children who were taught two actions. However, exploratory behaviours of the infants taught seven actions and of those in the free play condition decreased over time.

Cooper (2014) - Literacy/language: There were larger gains for comprehension and expressive vocabulary for the book sharing versus toy play condition (chi-square tests). Executive function: There was no group difference in focused attention (chi-square). 


\section{Interventions involving boardgames \& card games $(n=25)$}

A large proportion of studies identified in the search involved boardgames and card games, most of which targeted children's numeracy skills. These studies are summarised briefly in the table below but are not discussed in detail in the main report. Theses studies were deemed less relevant to the current review due to the limited degree of physical manipulation they afforded children.

Table E2. Characteristics of studies in involving board \& card games $(n=25)$

\begin{tabular}{|c|c|c|c|c|c|c|c|c|c|c|c|c|}
\hline ID & Location & $\begin{array}{c}\text { Sample } \\
\quad N\end{array}$ & Age & $\begin{array}{c}\text { Research } \\
\text { design }\end{array}$ & Setting & $\begin{array}{l}\text { Model of } \\
\text { delivery }\end{array}$ & $\begin{array}{c}\text { Adult } \\
\text { involved }\end{array}$ & $\begin{array}{c}N \\
\text { sessions }\end{array}$ & Materials & Intervention & Comparison(s) & $\begin{array}{c}\text { Main outcome } \\
\text { domains }\end{array}$ \\
\hline $\begin{array}{l}\text { Bengtson } \\
2013\end{array}$ & USA & 95 & $7-9$ & $\begin{array}{l}\text { QE, pre- } \\
\text { post }\end{array}$ & $\begin{array}{c}\text { School/ } \\
\text { classroom }\end{array}$ & Group & Unclear & $2-5$ & $\begin{array}{l}\text { Boardgame } \\
\text { \& puzzle }\end{array}$ & $\begin{array}{l}\text { Linear number } \\
\text { boardgame }\end{array}$ & $\begin{array}{l}\text { 1. Broken number line puzzle } \\
\text { 2. No-intervention }\end{array}$ & $\begin{array}{c}\text { Math/ } \\
\text { numeracy }\end{array}$ \\
\hline $\begin{array}{c}\text { Benzing } \\
2019\end{array}$ & Switzerland & 118 & $10-12$ & $\begin{array}{l}\text { RCT, pre- } \\
\text { post }\end{array}$ & $\begin{array}{l}\text { School/ } \\
\text { classroom }\end{array}$ & $\begin{array}{l}\text { Class / } \\
\text { group }\end{array}$ & Teacher & $11-20$ & Card games & $\begin{array}{l}\text { Executive function card } \\
\text { game training }\end{array}$ & Business-as-usual & $\begin{array}{l}\text { Executive } \\
\text { function }\end{array}$ \\
\hline $\begin{array}{l}\text { Bofferding } \\
\quad 2015\end{array}$ & USA & 92 & $4-6$ & $\begin{array}{l}\text { RCT, pre- } \\
\text { post }\end{array}$ & $\begin{array}{l}\text { School/ } \\
\text { classroom }\end{array}$ & Individual & Researcher & $2-5$ & Boardgame & $\begin{array}{l}\text { Linear number } \\
\text { boardgame }\end{array}$ & $\begin{array}{l}\text { Number games (e.g., counting } \\
\text { items, ordering cards) }\end{array}$ & $\begin{array}{l}\text { Math/ } \\
\text { numeracy }\end{array}$ \\
\hline $\begin{array}{c}\text { Cheung } \\
2017\end{array}$ & $\begin{array}{l}\text { Hong Kong } \\
\text { SAR, China }\end{array}$ & 88 & $4-6$ & $\begin{array}{l}\text { RCT, pre- } \\
\text { post }\end{array}$ & Home & Individual & $\begin{array}{l}\text { Parent/ } \\
\text { caregiver }\end{array}$ & $6-10$ & Boardgame & $\begin{array}{c}\text { Number boardgame \& } \\
\text { parent training }\end{array}$ & $\begin{array}{l}\text { 1. Number boardgame (no } \\
\text { parent training) } \\
\text { 2. Exercise books } \\
\text { 3. No-intervention }\end{array}$ & $\begin{array}{l}\text { Math/ } \\
\text { numeracy }\end{array}$ \\
\hline $\begin{array}{l}\text { Chituk } \\
2003\end{array}$ & USA & $41^{\mathrm{a}}$ & $4-6$ & $\begin{array}{l}\text { RCT, pre- } \\
\text { post }\end{array}$ & $\begin{array}{l}\text { School/ } \\
\text { classroom }\end{array}$ & $\begin{array}{l}\text { Class / } \\
\text { group }\end{array}$ & Researcher & $6-10$ & Boardgame & $\begin{array}{l}\text { Chutes \& Ladders } \\
\text { (counting) boardgame }\end{array}$ & $\begin{array}{l}\text { 1. Computerised Chutes \& } \\
\text { Ladders } \\
\text { 2. Counting objects } \\
\text { 3. No-intervention }\end{array}$ & $\begin{array}{l}\text { Math/ } \\
\text { numeracy }\end{array}$ \\
\hline $\begin{array}{l}\text { Drury } \\
2018\end{array}$ & USA & 56 & $4-6$ & $\begin{array}{l}\text { RCT, pre- } \\
\text { post }\end{array}$ & $\begin{array}{l}\text { School/ } \\
\text { classroom }\end{array}$ & Group & Researcher & $2-5$ & Card game & $\begin{array}{c}\text { Subitizing game: rapid } \\
\text { judgement (of number of } \\
\text { monster eyes) }\end{array}$ & $\begin{array}{l}\text { Artificial reality-based } \\
\text { subitizing game }\end{array}$ & $\begin{array}{l}\text { Math/ } \\
\text { numeracy }\end{array}$ \\
\hline $\begin{array}{c}\text { Dunbar } \\
2017\end{array}$ & Canada & 54 & $0-3$ & $\begin{array}{l}\text { RCT, pre- } \\
\text { post }\end{array}$ & $\begin{array}{l}\text { Childcare } \\
\text { centre }\end{array}$ & $\begin{array}{l}\text { Individual } \\
\text { or pairs }\end{array}$ & Researcher & $2-5$ & Boardgame & $\begin{array}{l}\text { Two-row linear number } \\
\text { boardgame }\end{array}$ & $\begin{array}{l}\text { 1. Single-row linear number } \\
\text { boardgame } \\
\text { 2. Colour boardgame } \\
\text { 3. No-intervention }\end{array}$ & $\begin{array}{l}\text { Math/ } \\
\text { numeracy }\end{array}$ \\
\hline $\begin{array}{c}\text { Elofsson } \\
2016\end{array}$ & Sweden & 114 & $4-6$ & $\begin{array}{l}\text { RCT, pre- } \\
\text { post }\end{array}$ & $\begin{array}{l}\text { School/ } \\
\text { classroom }\end{array}$ & Pairs & Researcher & $6-10$ & Boardgame & $\begin{array}{l}\text { Linear number } \\
\text { boardgame }\end{array}$ & $\begin{array}{l}\text { 1. Circular number } \\
\text { boardgame } \\
\text { 2. Number activities } \\
\text { 3. No-intervention }\end{array}$ & $\begin{array}{l}\text { Math/ } \\
\text { numeracy }\end{array}$ \\
\hline $\begin{array}{c}\text { Estrada-Plana } \\
2019\end{array}$ & Spain & $28^{b}$ & $7-9$ & $\begin{array}{l}\text { RCT, pre- } \\
\text { post }\end{array}$ & Lab & Group & Researcher & $2-5$ & $\begin{array}{l}\text { Commercial } \\
\text { boardgame }\end{array}$ & $\begin{array}{c}\text { Games targeting working } \\
\text { memory }\end{array}$ & Wait-list control & $\begin{array}{l}\text { Executive } \\
\text { function }\end{array}$ \\
\hline $\begin{array}{l}\text { Fokides } \\
2018\end{array}$ & Greece & 135 & $7-9$ & $\begin{array}{l}\text { QE, post- } \\
\text { only }\end{array}$ & $\begin{array}{l}\text { School/ } \\
\text { classroom }\end{array}$ & Pairs & Teacher & $6-10$ & Boardgame & $\begin{array}{l}\text { Paper-based 'Kodable' } \\
\text { game (see next box) }\end{array}$ & $\begin{array}{c}\text { 1. 'Kodable' on computer } \\
\text { tablet (basic programming } \\
\text { commands) } \\
\text { 2. Teaching with notes }\end{array}$ & $\begin{array}{l}\text { Other } \\
\text { (programming } \\
\text { skills) }\end{array}$ \\
\hline $\begin{array}{c}\text { Hawes } \\
2020\end{array}$ & Canada & 43 & $4-6$ & $\begin{array}{l}\text { RCT, pre- } \\
\text { post }\end{array}$ & $\begin{array}{l}\text { School/ } \\
\text { classroom }\end{array}$ & Pairs & Teacher & $2-5$ & Boardgame & $\begin{array}{l}\text { Linear number } \\
\text { boardgame }\end{array}$ & Linear colour boardgame & $\begin{array}{l}\text { Math/ } \\
\text { numeracy }\end{array}$ \\
\hline $\begin{array}{l}\text { Laski } \\
2014\end{array}$ & USA & 42 & $4-6$ & $\begin{array}{l}\text { RCT, pre- } \\
\text { post }\end{array}$ & $\begin{array}{l}\text { School/ } \\
\text { classroom }\end{array}$ & Individual & Researcher & $2-5$ & Boardgame & $\begin{array}{l}\text { Number grid boardgame } \\
\& \text { 'count on' aloud }\end{array}$ & $\begin{array}{l}\text { Number grid boardgame, \& } \\
\text { 'count from one' aloud }\end{array}$ & $\begin{array}{l}\text { Math/ } \\
\text { numeracy }\end{array}$ \\
\hline
\end{tabular}


PEDAL Report: PM scoping review

\begin{tabular}{|c|c|c|c|c|c|c|c|c|c|c|c|c|}
\hline $\begin{array}{l}\text { Nikiforidou } \\
\quad 2019\end{array}$ & Greece & 480 & $4-6$ & $\begin{array}{l}\text { QE, } \\
\text { during- } \\
\text { only }\end{array}$ & $\begin{array}{l}\text { School/ } \\
\text { classroom }\end{array}$ & Group & Researcher & 1 & Card game & $\begin{array}{l}\text { Animal card guessing } \\
\text { game (probabilistic) }\end{array}$ & $\begin{array}{c}\text { Computerised (PowerPoint) } \\
\text { guessing game }\end{array}$ & $\begin{array}{l}\text { Math/ } \\
\text { numeracy }\end{array}$ \\
\hline $\begin{array}{l}\text { Park } \\
2017\end{array}$ & $\begin{array}{l}\text { South } \\
\text { Korea }\end{array}$ & 57 & $10-12$ & $\begin{array}{l}\text { QE, pre- } \\
\text { post }\end{array}$ & $\begin{array}{l}\text { School/ } \\
\text { classroom }\end{array}$ & Group & Teacher & $11-20$ & $\begin{array}{l}\text { Commercial } \\
\text { boardgames }\end{array}$ & $\begin{array}{l}\text { Math (shape) games \& } \\
\text { teacher guidance }\end{array}$ & $\begin{array}{l}\text { 1. Free play (same games, no } \\
\text { guidance) } \\
\text { 2. Regular teaching }\end{array}$ & $\begin{array}{l}\text { Math/ } \\
\text { numeracy }\end{array}$ \\
\hline $\begin{array}{c}\text { Ramani } \\
2011\end{array}$ & USA & 88 & $4-6$ & $\begin{array}{l}\text { RCT, pre- } \\
\text { post }\end{array}$ & $\begin{array}{l}\text { School/ } \\
\text { classroom }\end{array}$ & Individual & Researcher & $2-5$ & Boardgame & $\begin{array}{l}\text { Linear number } \\
\text { boardgame (spin spinner, } \\
\text { move token, count aloud) }\end{array}$ & $\begin{array}{l}\text { 1. Circular number } \\
\text { boardgame } \\
\text { 2. Numerical activities }\end{array}$ & $\begin{array}{l}\text { Math/ } \\
\text { numeracy }\end{array}$ \\
\hline $\begin{array}{l}\text { Ramani } \\
2012 b\end{array}$ & USA & 105 & $4-6$ & $\begin{array}{l}\text { RCT, pre- } \\
\text { post }\end{array}$ & $\begin{array}{l}\text { School/ } \\
\text { classroom }\end{array}$ & Group & Teacher & $2-5$ & Boardgame & As above & Colour boardgame & $\begin{array}{l}\text { Math/ } \\
\text { numeracy }\end{array}$ \\
\hline $\begin{array}{l}\text { Ramani } \\
2020\end{array}$ & USA & 39 & $4-6$ & $\begin{array}{l}\text { RCT, pre- } \\
\text { post }\end{array}$ & Home & Individual & $\begin{array}{l}\text { Parent/ } \\
\text { caregiver }\end{array}$ & $11-20$ & Card game & $\begin{array}{l}\text { Magnitude comparison } \\
\text { game (dots \& numerals) }\end{array}$ & $\begin{array}{c}\text { Shape \& colour matching card } \\
\text { game }\end{array}$ & $\begin{array}{l}\text { Math/ } \\
\text { numeracy }\end{array}$ \\
\hline $\begin{array}{l}\text { Scalise } \\
2020\end{array}$ & USA & 76 & $4-6$ & $\begin{array}{l}\text { RCT, pre- } \\
\text { post }\end{array}$ & $\begin{array}{l}\text { School/ } \\
\text { classroom }\end{array}$ & Individual & Researcher & $2-5$ & Card game & As above & $\begin{array}{l}\text { 1. Numerical memory- } \\
\text { matching card game } \\
\text { 2. Shape \& colour matching } \\
\text { card game }\end{array}$ & $\begin{array}{l}\text { Math/ } \\
\text { numeracy, } \\
\text { executive } \\
\text { function }\end{array}$ \\
\hline $\begin{array}{c}\text { Siegler } \\
2009\end{array}$ & USA & 88 & $4-6$ & $\begin{array}{l}\text { RCT, pre- } \\
\text { post }\end{array}$ & $\begin{array}{l}\text { School/ } \\
\text { classroom }\end{array}$ & Individual & Researcher & $2-5$ & Board game & $\begin{array}{l}\text { Linear number } \\
\text { boardgame (spin spinner, } \\
\text { move token, count aloud) }\end{array}$ & $\begin{array}{l}\text { 1. Circular boardgame } \\
\text { 2. Number tasks }\end{array}$ & $\begin{array}{l}\text { Math/ } \\
\text { numeracy }\end{array}$ \\
\hline $\begin{array}{l}\text { Sonnenschein } \\
2016\end{array}$ & USA & 34 & $4-6$ & $\begin{array}{l}\text { RCT, pre- } \\
\text { post }\end{array}$ & Home & $\begin{array}{l}\text { Individual/ } \\
\text { pairs }\end{array}$ & $\begin{array}{l}\text { Parent/ } \\
\text { caregiver }\end{array}$ & $11-20$ & Boardgame & $\begin{array}{l}\text { Chutes \& Ladders } \\
\text { boardgame \& counting } \\
\text { aloud }\end{array}$ & $\begin{array}{l}\text { 1. Chutes \& Ladders (no } \\
\text { counting) } \\
\text { 2. CandyLand colour game } \\
\text { (no counting) }\end{array}$ & $\begin{array}{c}\text { Math/ } \\
\text { numeracy }\end{array}$ \\
\hline $\begin{array}{l}\text { Türkoğlu } \\
2019\end{array}$ & Turkey & 80 & $10-12$ & $\begin{array}{l}\text { QE, pre- } \\
\text { post }\end{array}$ & $\begin{array}{l}\text { School/ } \\
\text { classroom }\end{array}$ & $\begin{array}{l}\text { Individual/ } \\
\text { class/ } \\
\text { group }\end{array}$ & Teacher & $11-20$ & $\begin{array}{l}\text { Commercial } \\
\text { board \& } \\
\text { card games }\end{array}$ & $\begin{array}{l}\text { Games targeting cognitive } \\
\text { skills (e.g., reasoning, } \\
\text { linguistic, spatial) }\end{array}$ & No-intervention & Social-emotional \\
\hline $\begin{array}{l}\text { Vargianniti } \\
\quad 2019\end{array}$ & Greece & 43 & $10-12$ & $\begin{array}{l}\text { QE, pre- } \\
\text { post }\end{array}$ & $\begin{array}{l}\text { School/ } \\
\text { classroom }\end{array}$ & $\begin{array}{l}\text { Class/ } \\
\text { group }\end{array}$ & Unclear & 1 & Boardgame & $\begin{array}{l}\text { 'Geopoly' (Monopoly-like } \\
\text { game about Europe) }\end{array}$ & No-intervention & $\begin{array}{l}\text { Other (learning } \\
\text { retention - } \\
\text { geography) }\end{array}$ \\
\hline $\begin{array}{l}\text { Vogt } \\
2018\end{array}$ & Switzerland & 324 & $4-6$ & $\begin{array}{l}\text { RCT, pre- } \\
\text { post }\end{array}$ & $\begin{array}{l}\text { School/ } \\
\text { classroom }\end{array}$ & Group & Teacher & $>20$ & $\begin{array}{l}\text { Board \& } \\
\text { cards } \\
\text { games }\end{array}$ & $\begin{array}{l}\text { Math games (e.g., Shut the } \\
\text { Box, Lining-up the Fives) }\end{array}$ & $\begin{array}{l}\text { 1. Math training with PMs } \\
\text { (quantity, counting, numbers) } \\
\text { 2. Business-as-usual }\end{array}$ & $\begin{array}{c}\text { Math/ } \\
\text { numeracy }\end{array}$ \\
\hline $\begin{array}{l}\text { Whyte } \\
2008\end{array}$ & UK & 45 & $0-3$ & $\begin{array}{l}\text { RCT, pre- } \\
\text { post }\end{array}$ & $\begin{array}{l}\text { School/ } \\
\text { classroom }\end{array}$ & Group & Researcher & $2-5$ & Boardgame & $\begin{array}{l}\text { Linear number } \\
\text { boardgame (spin spinner, } \\
\text { move token, count aloud) }\end{array}$ & $\begin{array}{l}\text { 1. Linear colour boardgame } \\
\text { (speak colours aloud) } \\
\text { 2. Nonlinear number card } \\
\text { game }\end{array}$ & $\begin{array}{l}\text { Math/ } \\
\text { numeracy }\end{array}$ \\
\hline
\end{tabular}




\subsection{Appendix F: References of included studies}

Aleid, Mohammed Abdulaziz, E. (2015). Using peer tutoring and manipulatives to improve mathematics education in elementary schools in Saudi Arabia [Durham University]. http://etheses.dur.ac.uk

Alghazo, I., Alsawaie, O., \& Al-Awidi, H. (2010). Enhancing counting skills of preschoolers through the use of computer technology and manipulatives. International Journal of Learning, 17(9), 159176. https://doi.org/10.18848/1447-9494/cgp/v17i09/47227

Alshehri, S. (2017). The Comparison of Physical/Virtual Manipulative on Fifth-Grade Students' Understanding of Adding Fractions [University of Cincinnati]. In ProQuest Dissertations and Theses.

Bengtson, B. J. (2013). Changing mental representations using related physical models: The effects of analyzing number lines on learner internal scale of numerical magnitude. University of Minnesota.

Bennett, T. L. (2000). Teachers' use of children's literature, mathematics manipulatives, and scaffolding to improve preschool mathematics achievement: Does it work? (Issue December) [University of North Texas].

Benzing, V., Schmidt, M., Jäger, K., Egger, F., Conzelmann, A., \& Roebers, C. M. (2019). A classroom intervention to improve executive functions in late primary school children: Too "old" for improvements? British Journal of Educational Psychology, 89(2), 225-238. http://10.0.4.87/bjep.12232

Biazak, J. E., Marley, S. C., \& Levin, J. R. (2010). Does an activity-based learning strategy improve preschool children's memory for narrative passages? Early Childhood Research Quarterly, 25(4), 515-526. https://doi.org/10.1016/j.ecresq.2010.03.006

Boakes, N. J. (2009). Origami Instruction in the Middle School Mathematics Classroom: Its Impact on Spatial Visualization and Geometry Knowledge of Students. RMLE Online, 32(7), 1-12. https://doi.org/10.1080/19404476.2009.11462060

Bofferding, L., Hoffman, A., Suazo, E., \& Lisy, N. (2015). Number Line Estimation with Negatives. Proceedings of the 37th Annual Meeting of the North American Chapter of the International Group for the Psychology of Mathematics Education., 133-140.

Boyle, A. (2017). Developing Spatial Reasoning Skills: The Effects of Socialized Block Play. Pace University.

Brey, A. (2017). The effect of "Six Bricks" guided play on grade two learners' visual perception and reasoning abilities [Nelson Mandela Metropolitan University]. www.LEGOFoundation.com

Bugos, J. A., \& Demarie, D. (2017). The effects of a short-term music program on preschool children's executive functions. Psychology of Music, 45(6), 855-867. https://doi.org/10.1177/0305735617692666 
Bulunuz, M. (2013). Teaching science through play in kindergarten: DDDoes integrated play and science instruction build understanding? European Early Childhood Education Research Journal, 21(2), 226-249. https://doi.org/10.1080/1350293X.2013.789195

Burte, H., Gardony, A. L., Hutton, A., \& Taylor, H. A. (2017). Think3d!: Improving mathematics learning through embodied spatial training. Cognitive Research: Principles and Implications, 2(1). https://doi.org/10.1186/s41235-017-0052-9

Cakmak, S., Isiksal, M., \& Koc, Y. (2014). Investigating effect of origami-based instruction on elementary students spatial skills and perceptions. Journal of Educational Research, 107(1), 59-68. https://doi.org/10.1080/00220671.2012.753861

Casey, B. M., Andrews, N., Schindler, H., Kersh, J. E., Samper, A., \& Copley, J. (2008). The development of spatial skills through interventions involving block building activities. Cognition and Instruction, 26(3), 269-309. https://doi.org/10.1080/07370000802177177

Casey, B. M., Erkut, S., Ceder, I., \& Young, J. M. (2008). Use of a storytelling context to improve girls' and boys' geometry skills in kindergarten. Journal of Applied Developmental Psychology, 29(1), 29-48. https://doi.org/10.1016/j.appdev.2007.10.005

Cavanaugh, D. M., Clemence, K. J., Teale, M. M., Rule, A. C., \& Montgomery, S. E. (2017). Kindergarten Scores, Storytelling, Executive Function, and Motivation Improved through Literacy-Rich Guided Play. Early Childhood Education Journal, 45(6), 831-843. https://doi.org/10.1007/s10643-016-0832-8

Cheung, S. K., \& McBride, C. (2017). Effectiveness of Parent-Child Number Board Game Playing in Promoting Chinese Kindergarteners' Numeracy Skills and Mathematics Interest. Early $\begin{array}{lll}\text { Education and } \quad \text { Development, 28(5), } & \text { 572-589. }\end{array}$ https://doi.org/10.1080/10409289.2016.1258932

Chituk, R. A. (2003). Mathematics instruction and practice: Teaching young children with developmental delays. Fordham University.

Christakis, D. A., Zimmerman, F. J., \& Garrison, M. M. (2007). Effect of block play on language acquisition and attention in toddlers: A pilot randomized controlled trial. Archives of Pediatrics and Adolescent Medicine, 161(10), 967-971. https://doi.org/10.1001/archpedi.161.10.967

Clearfield, M. W. (2019). Play for Success: An intervention to boost object exploration in infants from low-income households. Infant Behavior and Development, 55(April), 112-122. https://doi.org/10.1016/j.infbeh.2019.03.001

Cobb, J. (2000). The Effects of an Early Intervention Program with Preservice Teachers as Tutors on the Reading Achievement of Primary Grade At Risk Children. Reading Horizons, 41(3), 155173.

Cooper, P. J., Vally, Z., Cooper, H., Radford, T., Sharples, A., Tomlinson, M., \& Murray, L. (2014). Promoting Mother-Infant Book Sharing and Infant Attention and Language Development in an Impoverished South African Population: A Pilot Study. Early Childhood Education Journal, 42(2), 143-152. https://doi.org/10.1007/s10643-013-0591-8 
Cramer, K. A., Post, T. R., \& Delmas, R. C. (2002). Initial fraction learning by fourth- And fifth-grade students: A comparison of the effects of using commercial curricula with the effects of using the rational number project curriculum. Journal for Research in Mathematics Education, 33(2), 111-144. https://doi.org/10.2307/749646

Dang, S., Ved, A., \& Vemuri, K. (2018). Geography map knowledge acquisition by solving a jigsaw map compared to self-Study: Investigating game based learning. International Journal of GameBased Learning, 8(2), 80-89. https://doi.org/10.4018/IJGBL.2018040107

Dejonckheere, P. J. N., De Wit, N., Van de Keere, K., \& Vervaet, S. (2016). Exploring the classroom: Teaching science in early childhood. International Electronic Journal of Elementary Education, 8(4), 537-558. https://doi.org/10.12973/eu-jer.5.3.149

Dennis, C. (2012). The effects of the use of manipulatives on the comprehension of math concepts among fifth-grade students. EBSCOhost [Northcentral University].

Dickinson, D. K., Collins, M. F., Nesbitt, K., Toub, T. S., Hassinger-Das, B., Hadley, E. B., Hirsh-Pasek, K., \& Golinkoff, R. M. (2019). Effects of Teacher-Delivered Book Reading and Play on Vocabulary Learning and Self-Regulation among Low-Income Preschool Children. Journal of Cognition and Development, 20(2), 136-164. https://doi.org/10.1080/15248372.2018.1483373

Drury Stotz, M. (2018). Creature Counting: The Effects of Augmented Reality on Perseverance and Early Numeracy Skills. Lehigh University.

Dunbar, K., Ridha, A., Cankaya, O., Jiménez Lira, C., \& LeFevre, J. A. (2017). Learning to Count: Structured Practice With Spatial Cues Supports the Development of Counting Sequence Knowledge in 3-Year-Old English-Speaking Children. Early Education and Development, 28(3), 308-322. https://doi.org/10.1080/10409289.2016.1210458

Eason, S. H., \& Ramani, G. B. (2020). Parent-Child Math Talk About Fractions During Formal Learning and Guided Play Activities. Child Development, 91(2), 546-562. https://doi.org/10.1111/cdev.13199

Eisen, S., \& Lillard, A. S. (2020). Learning from Apps and Objects: The Human Touch. Mind, Brain, and Education, 14(1), 16-23. https://doi.org/10.1111/mbe.12224

Elofsson, J., Gustafson, S., Samuelsson, J., \& Träff, U. (2016). Playing number board games supports 5year-old children's early mathematical development. Journal of Mathematical Behavior, 43, 134-147. https://doi.org/10.1016/j.jmathb.2016.07.003

Ermakova, A. V. (2016). One or more external representations: What is better for learning? Boston College.

Ferrara, K., Hirsh-Pasek, K., Newcombe, N. S., Golinkoff, R. M., \& Lam, W. S. (2011). Block Talk: Spatial Language During Block Play. Mind, Brain, and Education, 5(3), 143-151. https://doi.org/10.1111/j.1751-228X.2011.01122.x

Fisher, K. R. (2011). Exploring the mechanisms of guided play in preschoolers' developing geometric shape concepts (Issue AAT 3440076) [Temple University]. 
Fisher, K. R., Hirsh-Pasek, K., Newcombe, N., \& Golinkoff, R. M. (2013). Taking shape: Supporting preschoolers' acquisition of geometric knowledge through guided play. Child Development, 84(6), 1872-1878. https://doi.org/10.1111/cdev.12091

Fokides, E. (2018). Teaching basic programming concepts to young primary school students using tablets: Results of a pilot project. International Journal of Mobile and Blended Learning, 10(1), 34-47. https://doi.org/10.4018/IJMBL.2018010103

Fujimura, N. (2001). Facilitating children's proportional reasoning: A model of reasoning processes and effects of intervention on strategy change. Journal of Educational Psychology, 93(3), 589603. https://doi.org/10.1037/0022-0663.93.3.589

Gecu-Parmaksiz, Z., \& Delialioglu, O. (2019). Augmented reality-based virtual manipulatives versus physical manipulatives for teaching geometric shapes to preschool children. British Journal of Educational Technology, 50(6), 3376-3390. https://doi.org/10.1111/BJET.12740

Gecu-Parmaksiz, Z., \& Delialioğlu, Ö. (2018). The effect of augmented reality activities on improving preschool children's spatial skills. Interactive Learning Environments, 28(7), 876-889. https://doi.org/10.1080/10494820.2018.1546747

Goldstein, T. R., \& Lerner, M. D. (2018). Dramatic pretend play games uniquely improve emotional control in young children. Developmental Science, 21(4). https://doi.org/10.1111/desc.12603

Han, M., Moore, N., Vukelich, C., \& Buell, M. (2010). Does play make a difference? Effects of play intervention on at-risk preschoolers' vocabulary learning. American Journal of Play, 3(1), 82105.

Harn, P.-L., \& Bo, S.-H. (2019). The Effectiveness of Playful Positive Psychology Interventions with Six Bricks and DUPLO ${ }^{\circledR}$ Play Box for Taiwan Children on Emotional Adaptation. World Journal of Research and Review, 9(5), 4-8. https://doi.org/10.31871/wjrr.9.5.4

Hawes, Z., Cain, M., Jones, S., Thomson, N., Bailey, C., Seo, J., Caswell, B., \& Moss, J. (2020). Effects of a Teacher-Designed and Teacher-Led Numerical Board Game Intervention: A Randomized Controlled Study with 4- to 6-Year-Olds. Mind, Brain, and Education, 14(1), 71-80. https://doi.org/10.1111/mbe.12215

Hawes, Z., Moss, J., Caswell, B., Naqvi, S., \& MacKinnon, S. (2017). Enhancing Children's Spatial and Numerical Skills through a Dynamic Spatial Approach to Early Geometry Instruction: Effects of a 32-Week Intervention. Cognition and Instruction, 35(3), 236-264. https://doi.org/10.1080/07370008.2017.1323902

Horan, E., \& Carr, M. (2018). How much guidance do students need? An intervention study on kindergarten mathematics with manipulatives. International Journal of Educational Psychology, 7(3), 286-316. https://doi.org/10.17583/ijep.2018.3672

Hull, D. M., Hinerman, K. M., Ferguson, S. L., Chen, Q., \& Näslund-Hadley, E. I. (2018). Teacher-Led Math Inquiry: A Cluster Randomized Trial in Belize. Educational Evaluation and Policy Analysis, 40(3), 336-358. https://doi.org/10.3102/0162373718768430 
Jemutai, S., \& Webb, P. (2019). Effects of a 6 brick duplo block guided play intervention on pre-literate learners' visual perception. South African Journal of Childhood Education, 9(1), 1-8. https://doi.org/10.4102/sajce.v9i1.634

Krisztián, Á., Bernáth, L., Gombos, H., \& Vereczkei, L. (2015). Developing numerical ability in children with mathematical difficulties using origami. Perceptual and Motor Skills, 121(1), 233-243. https://doi.org/10.2466/24.10.PMS.121c16x1

Lane, H. B., Pullen, P. C., Hudson, R. F., \& Konold, T. R. (2009). Identifying essential instructional components of literacy tutoring for struggling beginning readers. Literacy Research and Instruction, 48(4), 277-297. https://doi.org/10.1080/19388070902875173

Laski, E. V., \& Siegler, R. S. (2014). Learning from number board games: You learn what you encode. Developmental Psychology, 50(3), 853-864. https://doi.org/10.1037/a0034321

Lazonder, A. W., \& Ehrenhard, S. (2014). Relative effectiveness of physical and virtual manipulatives for conceptual change in science: How falling objects fall. Journal of Computer Assisted Learning, 30(2), 110-120. https://doi.org/10.1111/jcal.12024

Li, Y., Huang, Z., Jiang, M., \& Chang, T. W. (2016). The Effect on Pupils' Science Performance and Problem-Solving Ability through Lego: An Engineering Design-based Modeling Approach. Educational Technology \& Society, 19(3), 1176-3647.

Estrada-Plana, V., Esquerda, M., Mangues, R., March-Llanes, J., \& Moya-Higueras, J. (2019). A Pilot Study of the Efficacy of a Cognitive Training Based on Board Games in Children with Attention-Deficit/Hyperactivity Disorder: A Randomized Controlled Trial. Games for Health Journal, 8(4), 265-274. https://doi.org/10.1089/g4h.2018.0051

Lu, S. J., Liu, Y. C., Chen, P. J., \& Hsieh, M. R. (2020). Evaluation of AR embedded physical puzzle game on students' learning achievement and motivation on elementary natural science. Interactive Learning Environments, 28(4), 451-463. https://doi.org/10.1080/10494820.2018.1541908

Martin, T., Svihla, V., \& Smith, C. P. (2012). The role of physical action in fraction learning. Journal of Education and Human Development, 5(1), 1-17.

Marulcu, I., \& Barnett, M. (2016). Impact of an engineering design-based curriculum compared to an inquiry-based curriculum on fifth graders' content learning of simple machines. Research in Science and Technological Education, 34(1), 85-104. https://doi.org/10.1080/02635143.2015.1077327

Mattoon, C., Bates, A., Shifflet, R., Latham, N., \& Ennis, S. (2015). Examining computational skills in Prekindergarteners: The effects of traditional and digital manipulatives in a prekindergarten classroom. Early Childhood Research and Practice, 17(1).

Mendiburo, M., \& Hasselbring, T. (2011). Technology's Impact on Fraction Learning: An experimental comparison of virtual and physical manipulatives. SREE Conference Report. 148, 148-162.

Moyer-Packenham, P. S., Baker, J., Westenskow, A., Anderson, K., Shumway, J., Rodzon, K., \& Jordan, K. (2013). A Study Comparing Virtual Manipulatives with Other Instructional Treatments in 
Third- and Fourth-Grade Classrooms. Journal of Education, 193(2), 25-39. https://doi.org/10.1177/002205741319300204

Newman, S. D., Hansen, M. T., \& Gutierrez, A. (2016). An fMRI Study of the Impact of Block Building and Board Games on Spatial Ability. Frontiers in Psychology, 7(AUG), 1278. https://doi.org/10.3389/fpsyg.2016.01278

Nikiforidou, Z. (2019). Probabilities and Preschoolers: Do Tangible Versus Virtual Manipulatives, Sample Space, and Repetition Matter? Early Childhood Education Journal, 47(6), 769-777. https://doi.org/10.1007/s10643-019-00964-2

Olkun, S. (2003). Comparing computer versus concrete manipulatives in learning 2D geometry. Journal of Computers in Mathematics and Science Teaching, 22, 43-56. https://doi.org/10.1501/0000984

Park, J., \& Lee, K. (2017). Using board games to improve mathematical creativity. International Journal of Knowledge and Learning, 12(1), 49-58. https://doi.org/10.1504/IJKL.2017.088182

Pirrone, C., Tienken, C. H., Pagano, T., \& Di Nuovo, S. (2018). The Influence of Building Block Play on Mathematics Achievement and Logical and Divergent Thinking in Italian Primary School Mathematics Classes. Educational Forum, 82(1), 40-58. https://doi.org/10.1080/00131725.2018.1379581

Portsmore, M. D. (2009). Exploring how experience with planning impacts first grade students' planning and solutions to engineering design problems. [Tufts University]. In Dissertation Abstracts International Section A: Humanities and Social Sciences.

Raimundo, R., Marques-Pinto, A., \& Lima, M. L. (2013). The effects of a social-emotional learning program on elementary school children: the role of pupil's characteristics. Psychology in the Schools, 50(2), 165-180. http://10.0.3.234/pits.21667

Ramani, G. B., \& Scalise, N. R. (2020). It's more than just fun and games: Play-based mathematics activities for Head Start families. Early Childhood Research Quarterly, 50, 78-89. https://doi.org/10.1016/j.ecresq.2018.07.011

Ramani, G. B., \& Siegler, R. S. (2011). Reducing the gap in numerical knowledge between low- and middle-income preschoolers. Journal of Applied Developmental Psychology, 32(3), 146-159. https://doi.org/10.1016/j.appdev.2011.02.005

Ramani, G. B., Siegler, R. S., \& Hitti, A. (2012). Taking it to the classroom: Number board games as a small group learning activity. Journal of Educational Psychology, 104(3), 661-672. https://doi.org/10.1037/a0028995

Sawyer, J. E., \& Goldstein, T. R. (2019). Can Guided Play and Storybook Reading Promote Children's Drawing Development? Empirical Studies of the Arts, 37(1), 32-59. https://doi.org/10.1177/0276237418777946

Scalise, N. R., Daubert, E. N., \& Ramani, G. B. (2020). Benefits of Playing Numerical Card Games on Head Start Children's Mathematical Skills. Journal of Experimental Education, 88(2), 200220. https://doi.org/10.1080/00220973.2019.1581721 
Schmitt, S. A., Korucu, I., Napoli, A. R., Bryant, L. M., \& Purpura, D. J. (2018). Using block play to enhance preschool children's mathematics and executive functioning: A randomized controlled trial. Early Childhood Research Quarterly, 44, 181-191. https://doi.org/10.1016/j.ecresq.2018.04.006

Schröder, E., Gredebäck, G., Gunnarsson, J., \& Lindskog, M. (2020). Play enhances visual form perception in infancy-an active training study. Developmental Science, 23(3). https://doi.org/10.1111/desc.12923

Siegler, R. S., \& Ramani, G. B. (2009). Playing Linear Number Board Games-But Not Circular OnesImproves Low-Income Preschoolers' Numerical Understanding. Journal of Educational Psychology, 101(3), 545-560. https://doi.org/10.1037/a0014239

Simoncini, K., Forndran, A., Manson, E., Sawi, J., Philip, M., \& Kokinai, C. (2020). The Impact of Block Play on Children's Early Mathematics Skills in Rural Papua New Guinea. International Journal of Early Childhood, 52(1), 77-93. https://doi.org/10.1007/s13158-020-00261-9

Sonnenschein, S., Metzger, S. R., Dowling, R., Gay, B., \& Simons, C. L. (2016). Journal of Applied Research on Children: Informing Policy for Children at Risk Extending an Effective Classroom-Based Math Board Game Intervention to Preschoolers' Homes. Journal of Applied Research on Children, 7(2).

Sophian, C. (2004). Mathematics for the future: developing a Head Start curriculum to support mathematics learning. Early Childhood Research Quarterly, 19(1), 59. http://10.0.3.248/j.ecresq.2004.01.015

Starkey, P., Klein, A., \& Wakeley, A. (2004). Enhancing young children's mathematical knowledge through a pre-kindergarten mathematics intervention. Early Childhood Research Quarterly, 19(1), 99-120. https://doi.org/10.1016/j.ecresq.2004.01.002

Taylor, F. M. (2001). Effectiveness of concrete and computer simulated manipulatives on elementary students' learning skills and concepts in experimental probability [University of Florida]. In ProQuest Dissertations and Theses (Vol. 34, Issue 3).

Taylor, H. A., \& Hutton, A. (2013). Think3d!: Training Spatial Thinking Fundamental to STEM Education. Cognition and Instruction, 31(4), 434-455. https://doi.org/10.1080/07370008.2013.828727

Thompson, A. C. (2012). The Effect of Enhanced Visualization Instruction on First Grade Students' Scores on the North Carolina Standard Course Assessment [Liberty University]. ProQuest.

Thompson, T. (2016). The effects of concrete, virtual, and multimodal tangram manipulatives on second grade elementary students' mathematics achievement and development of spatial sense: A convergent parallel mixed methods study. Oklahoma State University.

Tian, M., Luo, T., Ding, J., Wang, X., \& Cheung, H. (2021). Spatial Ability and Theory of Mind: A Mediating Role of Visual Perspective Taking. Child Development, 92(4), 1590-1604. https://doi.org/10.1111/cdev.13546

Toub, T. S., Hassinger-Das, B., Nesbitt, K. T., Ilgaz, H., Weisberg, D. S., Hirsh-Pasek, K., Golinkoff, R. M., Nicolopoulou, A., \& Dickinson, D. K. (2018). The language of play: Developing preschool 
vocabulary through play following shared book-reading. Early Childhood Research Quarterly, 45(August), 1-17. https://doi.org/10.1016/j.ecresq.2018.01.010

Türkoğlu, B. (2019). The effect of educational board games training programme on the social skill development of the fourth graders. In Elementary Education Online (Vol. 18, Issue 3, pp. 1326-1344). https://doi.org/10.17051/ilkonline.2019.612180

van Schijndel, T. J. P., Singer, E., van der Maas, H. L. J., \& Raijmakers, M. E. J. (2010). A sciencing programme and young children's exploratory play in the sandpit. European Journal of Developmental Psychology, 7(5), 603-617. https://doi.org/10.1080/17405620903412344

Vander Heyden, K. M., Huizinga, M., \& Jolles, J. (2017). Effects of a classroom intervention with spatial play materials on children's object and viewer transformation abilities. Developmental Psychology, 53(2), 290-305. https://doi.org/10.1037/dev0000224

Vargianniti, I., \& Karpouzis, K. (2019). Effects of game-based learning on academic performance and student interest. Lecture Notes in Computer Science: Vol. 11899 LNCS. https://doi.org/10.1007/978-3-030-34350-7_32

Verdine, B. N., Zimmermann, L., Foster, L., Marzouk, M. A., Golinkoff, R. M., Hirsh-Pasek, K., \& Newcombe, N. (2019). Effects of geometric toy design on parent-child interactions and spatial language. Early Childhood Research Quarterly, 46, 126-141. https://doi.org/10.1016/j.ecresq.2018.03.015

Vogt, F., Hauser, B., Stebler, R., Rechsteiner, K., \& Urech, C. (2018). Learning through play-pedagogy and learning outcomes in early childhood mathematics. European Early Childhood Education Research Journal, 26(4), 589-603. https://doi.org/10.1080/1350293X.2018.1487160

Watchorn, P. D. R. (2011). Improving Children's Understanding of Mathematical Equivalence. University of Alberta.

Weisberg, D. S., Ilgaz, H., Hirsh-Pasek, K., Golinkoff, R., Nicolopoulou, A., \& Dickinson, D. K. (2015). Shovels and swords: How realistic and fantastical themes affect children's word learning. Cognitive Development, 35, 1-14. https://doi.org/10.1016/j.cogdev.2014.11.001

Whyte, J. C., \& Bull, R. (2008). Number Games, Magnitude Representation, and Basic Number Skills in Preschoolers. Developmental Psychology, 44(2), 588-596. https://doi.org/10.1037/00121649.44.2.588

Willson-Quayle, A. (2001). The Effects of Child-Centered, Teacher-Directed, and Scaffolded Instruction on Low- Income, Latino Preschoolers' Task Performance, Motivation, and Private Speech. George Mason University.

Yuzawa, M., \& Bart, W. M. (2002). Young children's learning of size comparison strategies: Effect of origami exercises. Journal of Genetic Psychology, 163(4), 459-478. https://doi.org/10.1080/00221320209598696

Zacharia, Z. C., Loizou, E., \& Papaevripidou, M. (2012). Is physicality an important aspect of learning through science experimentation among kindergarten students? Early Childhood Research Quarterly, 27(3), 447-457. https://doi.org/10.1016/j.ecresq.2012.02.004 


\subsection{Appendix G: Potentially relevant studies excluded due to insufficient information}

Adedoja, G., Abidoye, J. A., \& Afolabi, A. K. (2013). Effects of Two Puzzle-Based Instructional Strategies on Primary School Pupils' Learning Outcomes in Social Studies in Ondo State, Nigeria. African Educational Research Journal, 1(2), 58-63.

Beuermann, D. W., Naslund-Hadley, E., Ruprah, I. J., \& Thompson, J. (2013). The pedagogy of science and environment: Experimental evidence from Peru. The Journal of Development Studies, 49(5), 719-736.

Bright, J. B. (2010). AMSTI mathematics in grades 4 and 5: Student achievement and teacher perceptions (Doctoral dissertation, University of Alabama Libraries).

Chang, W. L., Yuan, Y., Lee, C. Y., Chen, M. H., \& Huang, W. G. (2013). Using Magic Board as a teaching aid in third grader learning of area concepts. Journal of Educational Technology \& Society, 16(2), 163-173.

Klein, A., Starkey, P., \& DeFlorio, L. (2019). Improving the Mathematical Knowledge of At-Risk Preschool Children: Two Approaches to Intensifying Early Math Intervention. In Cognitive Foundations for Improving Mathematical Learning (pp. 215-245). Academic Press.

McBride Martin, A. E. (2006). The effects of professional development to create standards -based curriculum on student achievement in fourth and fifth grade mathematics classrooms (Order No. 3299648). Available from ProQuest Dissertations \& Theses A\&I. (305336543).

Mwaura, P. A., Sylva, K., \& Malmberg, L. E. (2008). Evaluating the Madrasa preschool programme in East Africa: a quasi-experimental study. International Journal of Early Years Education, 16(3), 237-255. 